Mathematik

\title{
Cohomogeneity one manifolds with positive euler characteristic
}

Inaugural-Dissertation zur Erlangung des Doktorgrades der Naturwissenschaften im Fachbereich Mathematik und Informatik der Mathematisch-Naturwissenschaftlichen Fakultät der Westfälischen Wilhelms-Universität Münster

vorgelegt von

Philipp Frank aus Heidelberg

$-2011-$ 
Dekan:

Erster Gutachter:

Zweiter Gutachter:

Tag der mündlichen Prüfung(en):

Tag der Promotion:
Prof. Dr. Matthias Löwe

Prof. Dr. Burkhard Wilking

Prof. Dr. Christoph Böhm

$\underline{07.07 .2011}$

$\underline{07.07 .2011}$ 


\section{Contents}

Introduction $\quad \mathbf{5}$

The result . . . . . . . . . . . . . . . . 7

1 Preliminaries $\quad 12$

1.1 Cohomogeneity one actions . . . . . . . . . . . . . . . 12

1.1.1 The noncompact case. . . . . . . . . . . . . . 12

1.1.2 The compact case. . . . . . . . . . . . . . . . 12

1.2 Equivalence, uniqueness of diagrams and primitivity . . . . . . 13

1.3 The topology . . . . . . . . . . . . . . . . . . . . . . 14

1.4 Properties of the isotropy groups . . . . . . . . . . . 16

1.5 Known classification results . . . . . . . . . . . . 17

1.5.1 Cohomogeneity one manifolds of low dimension . . . . 17

1.5.2 Cohomogeneity one manifolds with a fixed point . . . . 17

2 Proof of theorem $0.1 \quad 18$

3 The general procedure $\quad 20$

3.1 The Spin-groups . . . . . . . . . . . . . . . . . . . . . . . . 20

3.1 .1 Notation . . . . . . . . . . . . . . . 21

$4 \mathrm{G}=\mathrm{SU}(3) \quad \mathbf{2 2}$

$\begin{array}{lll}5 & \mathrm{G}=\mathrm{SU}(4) & 25\end{array}$

$6 \mathrm{G}=\mathrm{SU}(n), n \geq 5 \quad 30$

$\begin{array}{lll}7 \mathrm{G}=\mathrm{SO}(2 n+1), n \geq 3 & 37\end{array}$

$7.1 \mathrm{G}=\operatorname{Spin}(2 n+1), n \geq 3 \ldots \ldots \ldots 42$

$8 \mathrm{G}=\mathrm{Sp}(n), n \geq 2 \quad 48$

$9 \mathrm{G}=\mathrm{SO}(2 n), n \geq 4 \quad 58$

$9.1 \operatorname{Spin}(2 n), n \geq 4 \ldots \ldots \ldots \ldots$

$\begin{array}{ll}\text { A Tables } & 65\end{array}$

B Some computations $\quad 66$

B.1 The normalizer of $\mathrm{U}(n)$ in $\mathrm{SO}(2 n) \ldots \ldots \ldots 66$

B.2 Sphere actions of $\mathrm{U}(n) \ldots \ldots \ldots 66$

B.2.1 Actions of $\mathrm{U}(n+1)$ on spheres . . . . . . . . 66

B.2.2 Identifying the $\mathrm{S}^{1} \ldots \ldots \ldots$. . . . . . . 67 
B.2.3 Actions of $\mathrm{U}(n)$ on real projective spaces . . . . 68 


\section{Introduction}

Cohomogeneity one manifolds are Riemannian manifolds with an action of a lie group such that the orbit space is one dimensional. They can be seen as generalisations of homogeneous spaces, but offer a richer structure which has been used for explicit constructions in the past. In particular, Bergery used cohomogeneity one manifolds to construct invariant Einstein metrics, and later Bryant and Salamon found cohomogeneity one metrics with exceptional holonomy groups, which is not possible in the homogeneous case. For details and many references see e.g. Alekseevsky and Alekseevsky [1992], Alekseevsky and Podestá [1997], Alekseevsky and Alekseevsky [1993] and Ale [1992].

An interesting connection arises with nonnegative or positive sectional curvature. Most constructions of nonnegatively or positively curved manifolds arise from product and quotient constructions, starting with lie groups and their bi-invariant metric. Grove and Ziller found a large class of nonnegatively curved manifolds within the cohomogeneity one category in Grove and Ziller [2000]: If there are two orbits of codimension two, there is a metric of nonnegative sectional curvature (this in particular includes the principal L-bundles over $\mathrm{S}^{4}$ with $\mathrm{L}=\mathrm{SO}(3)$ or $\left.\mathrm{L}=\mathrm{SO}(4)\right)$. Later Grove and Ziller showed that every cohomogeneity one manifolds supports a metric of nonnegative Ricci curvature, and one of positive Ricci curvature if and only if the fundamental group is finite (Grove and Ziller [2002]). The naturally arising question if every cohomogeneity one manifold with finite fundamental group allows a metric of positive sectional curvature was answered negatively in Grove et al. [2006], where the Brieskorn variety is shown to be a counter example.

This work is concerned with the classification of cohomogeneity one manifolds with positive euler characteristic where the acting group $\mathrm{G}$ is classical simple. The first result is the following theorem, which hints at the importance of the classification result. The action is called primitive if there is no subgroup $\mathrm{L} \subset \mathrm{G}$ with a $\mathrm{G}$-equivariant map $M \rightarrow \mathrm{G} / \mathrm{L}$.

Theorem 0.1. Suppose the compact, connected lie group $\mathrm{G}$ acts primitively on the manifold $M$ with positive euler characteristic such that $M / G=[0,1]$. Suppose there is no normal subgroup of $\mathrm{G}$ that acts orbit equivalently, and that $\mathrm{G}$ is not simple. Then one of the following applies:

1. The action of $\mathrm{G}$ is equivalent to a cohomogeneity one action of a rank 1 symmetric space.

2. $\mathrm{G}$ is covered by $\mathrm{G}^{\prime} \times \mathrm{S}^{3}$ where $\mathrm{G}^{\prime}$ is simple and one of the singular orbits has codimension 3. 
While the classification result itself was moved to its own subsection within the introduction (due to the vast array of tables required), it should be noted that the number of examples in each dimension is finite. We know of no abstract reason for this. For each simple classical lie group $G$ we will give a table of cohomogeneity one G-manifolds of positive euler characteristic in the following subsection. We will prove theorem 0.1 in section 2 , after we have established the basics and notation in section 1. Because of the diagram-chase like qualities of the classification, we give an overview over the general procedure in section 3 . In sections 4-9, the actual classification is carried out.

This classification would not have been possible without my advisor Burkhard Wilking, who I am indebted to for constant support throughout the years. I'd also like to thank Wolfgang Ziller for his help during my visit in 2007. 


\section{The result}

Each simply connected, primitive cohomogeneity one G-manifolds of positive euler characteristic, where $G$ is a classical simple group acting almost effectively, is, up to equivalence (that is, up to an outer automorphism of $\mathrm{G}$, up to G-equivariance) one of the following:

- One of the classified cohomogeneity one manifolds of dimension less or equal to 7 (see 1.5.1 on page 17).

- One of the actions with a fixed point described in 1.5.2 on page 17

- Contained in the following tables.

The entries of the following tables are of the form $\mathrm{H} \subset \mathrm{K}_{-}, \mathrm{K}_{+}$, where $\mathrm{K}_{+}$ has maximal rank in $\mathrm{G}$, and $\mathrm{K}_{ \pm} / \mathrm{H}$ are spheres. The manifolds $M$ is then equivalent to

$$
\mathrm{G} \times \mathrm{K}_{-} D^{l_{-}+1} \cup \mathrm{G} \times \mathrm{K}_{+} D^{l_{+}+1}
$$

where $D^{l_{ \pm}+1}$ is the unit ball with boundary $\partial D^{l_{-} \pm+1}=\mathrm{K}_{ \pm} / \mathrm{H}$, where the action of $\mathrm{K}_{ \pm}$is extended linearly from the sphere $\mathrm{K}_{ \pm} / \mathrm{H}$, and $\mathrm{G} \times \mathrm{K}_{ \pm} D^{l_{ \pm}+1}$ is the quotient of $\mathrm{G} \times D^{l_{ \pm}+1}$ by the diagonal action of $\mathrm{K}_{ \pm}$(see 1.1 on page 12 for more details).

The tables for the Spin-groups (including SU(4) $\simeq$ Spin(6)) only list the effective actions (the non-effective ones are listed for the SO-groups), but those are given as $\pi(\mathrm{H}) \subset \pi\left(\mathrm{K}_{-}\right), \pi\left(\mathrm{K}_{+}\right)$, where $\pi: \operatorname{Spin}(n) \rightarrow \mathrm{SO}(n)$ is the projection. The diagram of the original action can be reconstructed as $\mathrm{H}=\pi^{-1}(\pi(\mathrm{H}))_{0}, \mathrm{~K}_{ \pm}=\pi^{-1}\left(\pi\left(\mathrm{K}_{ \pm}\right)\right.$) (see section 3 on page 20 and 3.1 on page 20) in those cases.

- $\mathrm{G}=\mathrm{SU}(3)$

\begin{tabular}{|c|}
\hline $\mathrm{S}^{1} \subset \mathrm{SU}(2), \mathrm{S}(\mathrm{U}(1) \mathrm{U}(2))$ \\
\hline $\mathrm{S}^{1} \subset \mathrm{S}(\mathrm{U}(2) \mathrm{U}(1)), \mathrm{S}(\mathrm{U}(1) \mathrm{U}(2))$ \\
\hline $\mathrm{S}^{1} \subset \mathrm{SO}(3), \mathrm{S}(\mathrm{U}(1) \mathrm{U}(2))$ \\
\hline $\mathrm{S}^{1} \subset \mathrm{SO}(3), \mathrm{T}^{2}$ \\
\hline $\mathbb{Z}_{3} \mathrm{SO}(3) \subset \mathbb{Z}_{3} \mathrm{SO}(3), \mathrm{T}^{2}$ \\
\hline
\end{tabular}


- $\mathrm{G}=\mathrm{SO}(6)$

\begin{tabular}{|c|}
\hline $\mathrm{SO}(4) \subset \mathrm{SO}(5), \mathrm{SO}(2) \mathrm{SO}(4)$ \\
\hline $\mathbb{Z}_{2} \mathrm{SO}(4) \subset \mathbb{Z}_{2} \mathrm{SO}(5), \mathrm{SO}(2) \mathrm{SO}(4)$ \\
\hline $\mathrm{SO}(2) \mathrm{SO}(3) \subset \mathrm{SO}(3) \mathrm{SO}(3), \mathrm{SO}(2) \mathrm{SO}(4)$ \\
\hline $\mathrm{SO}(2) \mathrm{SO}(2) \subset \mathrm{SO}(2) \mathrm{SO}(3), \mathrm{U}(2) \mathrm{SO}(2)$ \\
\hline $\mathrm{U}(2) \subset \mathrm{SO}(4), \mathrm{U}(3)$ \\
\hline $\mathrm{T}^{2} \subset \mathrm{SO}(3) \mathrm{SO}(2), \mathrm{SO}(2) \mathrm{U}(2)$ \\
where $\mathrm{T}^{2}=\left\{\operatorname{diag}\left(z_{1}, 1,1, z_{2}\right)\right\}$ \\
\hline
\end{tabular}

- $\mathrm{G}=\mathrm{SU}(4)$

\begin{tabular}{|c|}
\hline $\begin{array}{c}\mathrm{S}^{1} \mathrm{SU}(2) \subset \mathrm{S}(\mathrm{U}(2) \mathrm{U}(2)), \mathrm{S}(\mathrm{U}(1) \mathrm{U}(3)) \\
\text { where } \mathrm{S}^{1}=\left\{\operatorname{diag}\left(\bar{z}^{2}, z^{4}, \bar{z}, \bar{z}\right)\right\} \subset N(\mathrm{SU}(2))\end{array}$ \\
\hline $\begin{array}{c}\mathrm{S}^{1} \mathrm{SU}(2) \subset \mathrm{S}(\mathrm{U}(2) \mathrm{U}(2)), \mathrm{S}(\mathrm{U}(1) \mathrm{U}(3)) \\
\text { where } \mathrm{S}^{1}=\left\{\operatorname{diag}\left(\bar{z}^{2}, 1, z, z\right)\right\} \subset N(\mathrm{SU}(2))\end{array}$ \\
\hline $\mathrm{S}^{1} \subset \sigma(\mathrm{S}(\mathrm{U}(1) \mathrm{U}(3))), \mathrm{S}(\mathrm{U}(1) \mathrm{U}(3))$ \\
where $\mathrm{S}^{1}=\{\operatorname{diag}(\bar{z}, z, 1,1)\}$ and $\sigma$ exchanges the first two coordinates \\
\hline
\end{tabular}


- $\mathrm{G}=\mathrm{SU}(n), n \geq 5$

$$
\mathrm{S}^{1} \mathrm{SU}(n-2) \subset \sigma(\mathrm{U}(n-1)), \mathrm{U}(n-1)
$$

where $\mathbf{S}^{1}=\{\operatorname{diag}(\bar{z}, z, 1, \ldots, 1)\}$ and $\sigma$ exchanges first two coordinates

$$
\mathrm{S}^{1} \mathrm{SU}(n-2) \subset \mathrm{S}(\mathrm{U}(2) \mathrm{U}(n-2)), \mathrm{U}(n-1)
$$

where $\mathrm{S}^{1}=\left\{\operatorname{diag}\left(\bar{z}^{n-2}, z^{n-2}, \bar{z}, \ldots, \bar{z}\right)\right\} \subset N(\operatorname{SU}(n-2))$

$$
\mathrm{S}^{1} \mathrm{SU}(n-2) \subset \mathrm{S}(\mathrm{U}(2) \mathrm{U}(n-2)), \mathrm{U}(n-1)
$$

where $\mathrm{S}^{1}=\left\{\operatorname{diag}\left(\bar{z}^{n-2}, 1, z, \ldots, z\right)\right\} \subset N(\mathrm{SU}(n-2))$

$$
\mathrm{S}^{1} \mathrm{SU}(n-2) \subset \mathrm{SU}(2) \mathrm{SU}(n-2), \mathrm{U}(n-1)
$$

where $\mathrm{S}^{1}=\{\operatorname{diag}(\bar{z}, z, 1, \ldots, 1)\} \subset N(\mathrm{SU}(n-2))$

$$
\mathrm{S}^{1} \mathrm{SU}\left(n_{1}-1\right) \mathrm{SU}\left(n_{2}\right) \subset \mathrm{S}\left(\mathrm{U}\left(n_{1}-1\right) \mathrm{U}\left(n_{2}+1\right)\right), \mathrm{S}\left(\mathrm{U}\left(n_{1}\right) \mathrm{U}\left(n_{2}\right)\right)
$$

where $S^{1}=\left\{\operatorname{diag}(\underbrace{\bar{z}^{n_{2}}, \ldots, \bar{z}^{n_{2}}}_{\times n_{1}-1}, 1, \underbrace{z^{n_{1}-1}, \ldots, z^{n_{1}-1}}_{\times n_{2}})\right.$

and $\mathrm{SU}\left(n_{1}-1\right) \mathrm{SU}\left(n_{2}\right) \subset \mathrm{H}$ acts trivially on $\mathbb{C} n_{1}\left(n_{1}+n_{2}=n-1, n_{1}, n_{2}>1\right)$

- $\mathrm{G}=\mathrm{SO}(2 n+1), n \geq 3$

\begin{tabular}{|c|}
\hline $\mathrm{SO}(2 n-1) \subset \mathrm{SO}(2) \mathrm{SO}(2 n-1), \mathrm{SO}(2 n)$ \\
\hline $\mathrm{O}(2 n-1) \subset \mathrm{SO}(2) \mathrm{SO}(2 n-1), \mathrm{O}(2 n)$ \\
\hline $\mathrm{SO}\left(2 n_{1}+1\right) \mathrm{SO}\left(2 n_{2}-1\right) \subset \mathrm{SO}\left(2 n_{1}+2\right) \mathrm{SO}\left(2 n_{2}-1\right), \mathrm{SO}\left(2 n_{1}+1\right) \mathrm{SO}\left(2 n_{2}\right)$ \\
where $n_{1}+n_{2}=n$
\end{tabular}


- $\mathrm{G}=\operatorname{Spin}(2 n+1), n \geq 3$

\begin{tabular}{c}
\hline $\mathrm{S}_{k}^{1} \mathrm{SU}(2) \subset \mathrm{U}(3), \mathrm{SO}(2) \mathrm{SO}(5), n=3$ \\
where $\mathrm{S}_{k}^{1}=\left\{\operatorname{diag}\left(z^{2}, z^{k}, z^{k}, 1\right)\right\}, k=1,-3$ and \\
$\mathrm{SU}(2)$ acts trivially on $\mathbb{R} e_{1}, \mathbb{R} e_{2}, \mathbb{R} e_{7}$ \\
\hline $\mathrm{SU}(2) \mathrm{S}_{\mathrm{H}}^{1} \mathrm{SU}(2) \subset \mathrm{U}(2) \mathrm{SO}(5), \mathrm{SO}(5) \mathrm{U}(2), n=4$ \\
where $\mathrm{S}_{\mathrm{H}}^{1}=\left\{\operatorname{diag}\left(z^{l_{1}}, z^{l_{1}}, 1, z^{l_{2}}, z^{l_{2}}\right)\right\}$ and ${ }_{1}=2, l_{2}=1$ or $l_{1}=1=l_{2}$. \\
\hline
\end{tabular}

- $\mathrm{G}=\operatorname{Sp}(n), n \geq 2$

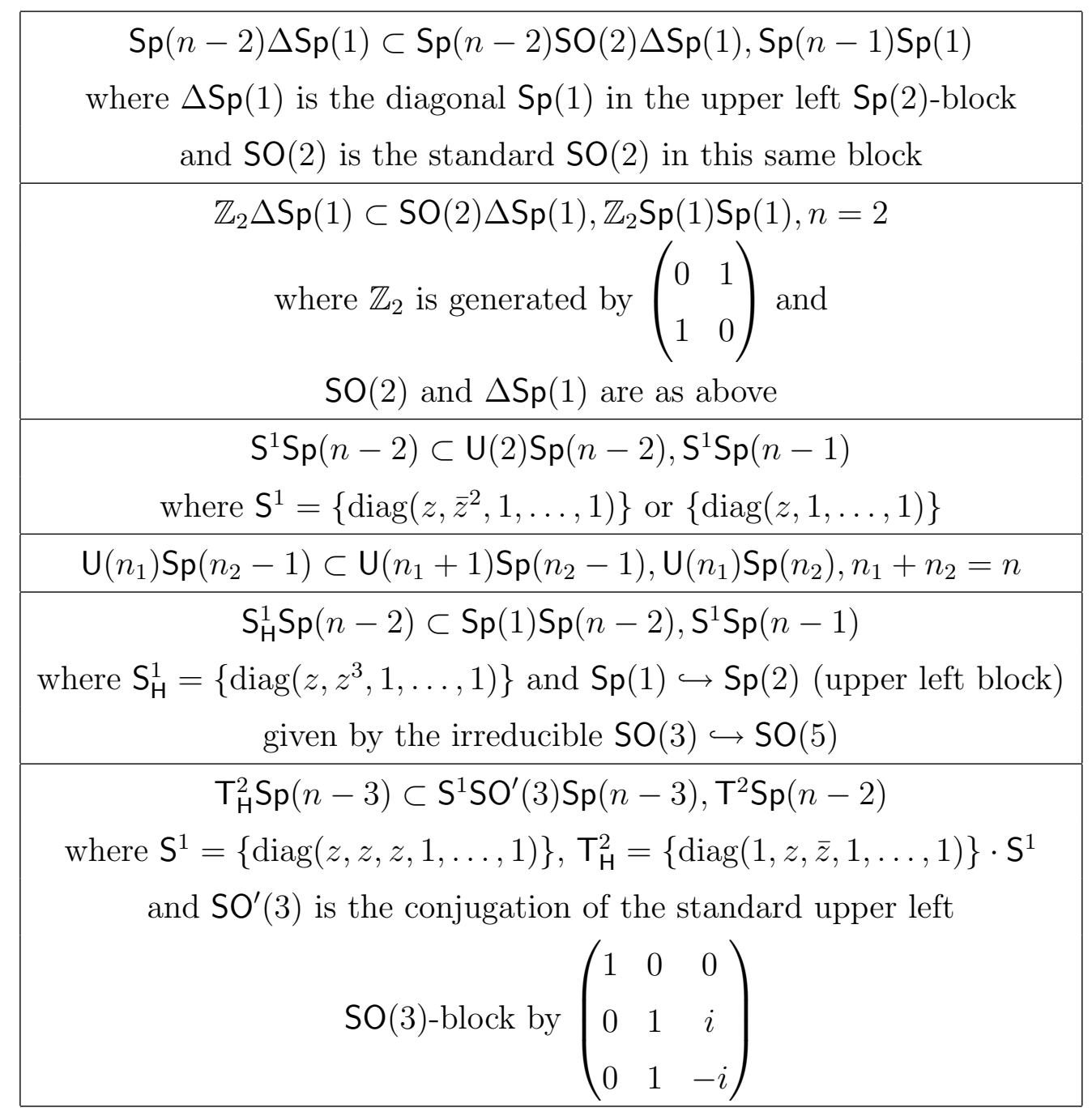


- $\mathrm{G}=\mathrm{SO}(2 n), n \geq 4$

\begin{tabular}{|c|}
\hline $\mathrm{S}^{1} \mathrm{SU}(n-1) \subset \sigma(\mathrm{U}(n)), \mathrm{U}(n)$ \\
where $\mathrm{S}^{1}=\{\operatorname{diag}(z, 1, \ldots, 1)\}$ or $\mathrm{S}^{1}=\{\operatorname{diag}(1, z, \ldots, z)\}$ and \\
$\sigma$ is conjugation by $\operatorname{diag}(-1,1, \ldots, 1)$
\end{tabular}




\section{Preliminaries}

In this section, we will cover the basics of cohomogeneity one manifolds, and list the properties most usefull to us, especially those connected to the topology of $M$. We will also treat some properties of representations of certain simple lie groups, which we will make use of later in the classification.

\subsection{Cohomogeneity one actions}

Suppose a compact Lie group $\mathrm{G}$ acts on the manifold $M$ such there is a 1codimensional orbit G/H. It is known (see Bredon [1972] or Mostert [1957]) that $M / \mathrm{G}$ is homeomorphic to either $\mathrm{S}^{1}$ or $[-1,1]$ in the compact case, or $\mathbb{R}$ or $[0,1)$ in the noncompact case. The structure of all cases can be found in the pioneer work of Mostert Mostert [1957], and also (in more detail) in various papers by Alekseevsky, see e.g. Alekseevsky and Alekseevsky [1992]. We list the most important properties:

\subsubsection{The noncompact case.}

If $M / \mathrm{G}=\mathbb{R}$, all orbits are regular and $M$ is actually a fibre bundle over $M / \mathrm{G}$ with fibre $\mathrm{G} / \mathrm{H}$, which is neccessarily trivial, since $\mathbb{R}$ is contractible. Therefore, $M$ is $\mathrm{G}$-equivalent to $\mathrm{G} / \mathrm{H} \times \mathbb{R}$. In the case $M / \mathrm{G}=[0,1)$, there is exactly one singular orbit, say $G / K$ (where $K \supset H$ ). Then $K / H$ is a sphere $\mathrm{S}^{m} \subset \mathbb{R}^{m+1}$, and the action of $\mathrm{K}$ on $\mathrm{S}^{m}$ can be extended linearly to $\mathbb{R}^{m+1}$. $M$ is $\mathrm{G}$-equivalent to $\mathrm{G} \times{ }_{\mathrm{K}} \mathbb{R}^{m+1}$, on which $\mathrm{G}$ acts by left translation on the first factor.

\subsubsection{The compact case.}

If $M / \mathrm{G}=\mathrm{S}^{1}$, all orbits are regular and $M$ is a fibre bundle over $\mathrm{S}^{1}$ with fibre $\mathrm{G} / \mathrm{H}$. It's easily seen by the homotopy sequence of that bundle that $\pi_{1}(M)$ is infinite in this case. Also we have $\chi(M)=0$, so we will not be concerned with this case.

The most interesting case is $M / G=[-1,1]$, which is the one we are concerned with in this paper, and which we therefore lay out in more detail. Choose any G-invariant Riemannian metric on $M$. There are two singular orbits, so choose a minimal geodesic $c:[-1,1] \rightarrow M$ between them, such that $\pi \circ c=i d_{[-1,1]}$ for the projection $\pi: M \rightarrow[-1,1]$. Denote the isotropy groups by $H=\mathrm{G}_{c(0)}$ and $K_{ \pm}=\mathrm{G}_{c( \pm 1)}$. By the slice theorem (see e.g. Bredon [1972]) there are tubular neighbourhoods $\mathrm{G} \times \mathrm{K}_{ \pm} D_{ \pm}$of the singular orbits $\mathrm{K}_{ \pm}$, where $D_{ \pm}$is the disc of radius 1 normal to the singular orbit $\mathrm{K}_{ \pm}$. Since the 
action of $\mathbf{G}$ on $M$ has cohomogeneity one, the action of $\mathrm{K}_{ \pm}$on $\mathrm{S}^{l_{ \pm}}=\partial D_{ \pm}$is transitive, with isotropy group $\mathrm{H}$. Now $M$ is constructed from the tubular neighbourhoods by gluing them along their common boundary $\pi^{-1}(0) \simeq$ $\mathrm{G} / \mathrm{H}$ :

$$
M \simeq \mathrm{G} \times \mathrm{K}_{-} D_{-} \cup_{\mathrm{G} / \mathrm{H}} \mathrm{G} \times \mathrm{K}_{+} D_{+}
$$

This uniquely determines $M$ in terms of the groups $\mathrm{G} \supset \mathrm{K}_{-}, \mathrm{K}_{+} \supset \mathrm{H}$ : The action of $\mathrm{K}_{ \pm}$on the sphere $\mathrm{S}^{l_{ \pm}}=\mathrm{K}_{ \pm} / \mathrm{H}$ is linear and can therefore be extended to an action on the ball $D_{ \pm}$, which in turn allows $M$ be constructed as above. Note that the only condition imposed besides the inclusions is that $\mathrm{K}_{ \pm} / \mathrm{H}$ is a sphere of any dimension. We call the collection of groups $\left\{\mathrm{G}, \mathrm{K}_{-}, \mathrm{K}_{+}, \mathrm{H}\right\}$ the group diagram of $M$, where it is understood that $\mathrm{K}_{ \pm} \subset \mathrm{G}$ and $\mathrm{H} \subset \mathrm{K}_{ \pm}$. The group diagram is of course not uniquely determined by $M$, since we did not assume effectiveness of the action of $\mathrm{G}$ : If $\mathrm{G} \supset \mathrm{K}_{-}, \mathrm{K}_{+} \supset \mathrm{H}$ is the group diagram of an ineffective cohomogeneity one manifold with ineffective kernel $H^{\prime}$, the effective version of the $G$ action has group diagram $G / H^{\prime} \supset$ $\mathrm{K}_{-} / \mathrm{H}^{\prime}, \mathrm{K}_{+} / \mathrm{H} \supset \mathrm{H} / \mathrm{H}^{\prime}$. But even effective group diagrams are not uniquely determined by $M$ (e.g., spheres can be represented by many such diagrams).

\subsection{Equivalence, uniqueness of diagrams and primitiv- ity}

For the remainder of this work, we assume that we are in the second case described in 1.1.2 on the previous page, and we use the notation introduced there.

The classification of cohomogeneity one manifolds will be done up to equvariant diffeomorphism: If $M_{1}, M_{2}$ carry a cohomogeneity one action of $\mathrm{G}$, we will call $M_{1}$ and $M_{2} \mathrm{G}$-equivariantly diffeomorphic if there is a diffeomorphism $\psi: M_{1} \rightarrow M_{2}$ such that $\psi(g p)=g \psi(p)$ for all $g \in \mathrm{G}, p \in M_{1}$. This will determine the group diagram of a cohomogeneity one G-manifold $M$ up to the following operations (see Grove et al. [2008]):

- Switching $\mathrm{K}_{-}$and $\mathrm{K}_{+}$

- Conjugating each group of the group diagram with an element of $\mathrm{G}$

- Replacing $\mathrm{K}_{-}$by $a \mathrm{~K}_{-} a^{-1}$ for some $a \in N(\mathrm{H})_{0}$, the unity component of the normalizer of $\mathrm{H}$ in $\mathrm{G}$, while keeping $\mathrm{H}$ and $\mathrm{K}_{+}$unmodified.

Of course, the classification works the other way around: We will classify the possible diagrams up to the listed operations and therefore get a list for the equivariant diffeomorphism types of cohomogeneity one G-manifolds for 
a fixed lie group G. We will not classify cohomogeneity one manifolds up to equivariant diffeomorphism, but up to a slightly coarser equivalence relation:

Definition 1.1. Two cohomogeneity one G-manifolds $M_{1}, M_{2}$ are called equivalent, if they are equivariantly diffeomorphic up to a automorphism of $\mathrm{G}$, i.e. if there is an automorphism $\varphi$ of $\mathrm{G}$ and a diffeomorphism $\psi: M_{1} \rightarrow M_{2}$ such that $\psi(g p)=\varphi(g) \psi(p)$ for all $g \in \mathrm{G}, p \in M_{1}$.

It is clear that applying an automorphism of $\mathrm{G}$ to all groups in the diagram of a cohomogeneity one manifold yields an equivalent cohomogeneity one manifold.

From a diagram $\mathrm{G} \supset \mathrm{K}_{-}, \mathrm{K}_{+} \supset \mathrm{H}$ and an embedding $\mathrm{G} \hookrightarrow \mathrm{G}^{\prime}$ we get a cohomogeneity one $G^{\prime}$-manifold from the diagram $G^{\prime} \supset K_{-}, K_{+} \supset H$. To further diminish the items of the classification we will require minimality with respect to this extension process:

Definition 1.2. A comohogeneity one G-manifold $M$ is called primitive, if for every group diagram $\mathrm{G} \supset \mathrm{K}_{-}, \mathrm{K}_{+} \supset \mathrm{H}$ of $M$ there is no subgroup $\mathrm{L} \subset \mathrm{G}$ such that $\mathrm{K}_{-}, \mathrm{K}_{+} \subset \mathrm{L}$.

Nonprimitivity of $M$ is equivalent to the existence of a G-equivariant map $M \rightarrow \mathrm{G} / \mathrm{L}$ for some subgroup $\mathrm{L} \subsetneq \mathrm{G}$. In this case, $M$ is equivalent to $\mathrm{G} \times \mathrm{L} N$, where $N$ is the cohomogeneity one manifold determined by the group diagram $\mathrm{L} \supset \mathrm{K}_{-}, \mathrm{K}_{+} \supset \mathrm{H}$ (see Alekseevsky and Alekseevsky [1992]). The action of $\mathrm{L}$ on $N$ does not need to be effective, even if the one of $\mathrm{G}$ on $M$ is.

As a motivation for studying primitive G-manifolds, note that if $N$ has non-negative curvature, so has $G \times_{\mathrm{L}} N$. The technical value of primitivity lies mostly in the following lemma, taken from Grove et al. [2008]:

Lemma 1.3. Assume the primitive cohomogeneity one action of $\mathrm{G}$ on $M$ is effective (almost effective). Then the intersection $\mathrm{H}_{-} \cap \mathrm{H}_{+}$of the ineffective kernels $\mathrm{H}_{ \pm}$of $\mathrm{K}_{ \pm} / \mathrm{H}$ is trivial (finite).

\subsection{The topology}

The description of $M$ as a double disc bundle in 1.1.2 on page 12 of course allows a computation of the fundamental group in terms of the group diagram. Vice versa, assuming $M$ being simply connected, one arrives at the following properties of the groups involved:

Proposition 1.4 (Grove et al. [2008]). Let $M$ be a simply connected cohomogeneity one G-manifold. Then there are no exceptional orbits, and, in the notation of 1.1.2 on page 12, we have $l_{ \pm} \geq 1$, that is, $\operatorname{dim} \mathrm{K}_{ \pm}>\operatorname{dim} \mathrm{H}$. 
A more direct computation using van Kampen's theorem, carried out in Hoelscher [2007], yields the following usefull properties:

Proposition 1.5. With the assumptions and notation of 1.4 on the preceding page:

- If $l_{+}>1$ and $l_{-} \geq 1$, then $\mathrm{K}_{-}$is connected

- If both $l_{ \pm}>1$, then all of $\mathrm{H}, \mathrm{K}_{-}, \mathrm{K}_{+}$are connected

We will utilize this proposition frequently without further mention: The only way the group $\mathrm{K}_{+}$can be non-connected is when the "other sphere" $\mathrm{K}_{-} / \mathrm{H}$ is 1 -dimensional.

Another result concerns the euler characteristic of $M$ (Alekseevsky and Podestá [1997]). Since $\mathrm{G} \times \mathrm{K}_{ \pm} D_{ \pm}$contains the singular orbit $\mathrm{G} / \mathrm{K}_{ \pm}$as a deformation rectract, the Mayer-Vietoris exact sequence can be applied to obtain the following exact sequence:

$$
\ldots \rightarrow H^{i}(M) \rightarrow H^{i}\left(\mathrm{~K}_{-}\right) \oplus H^{i}\left(\mathrm{~K}_{+}\right) \rightarrow H^{i}(\mathrm{G} / \mathrm{H}) \rightarrow H^{i+1}(M) \rightarrow \ldots
$$

This allows the following corollary:

Corollary 1.6. Let $M$ be a cohomogeneity one G-manifold with orbit space $[-1,1]$. Using notation from 1.1.2 on page 12, the euler characteristic of $M$ is given by

$$
\chi(M)=\chi\left(\mathrm{G} / \mathrm{K}_{-}\right)+\chi\left(\mathrm{G} / \mathrm{K}_{+}\right)-\chi(\mathrm{G} / \mathrm{H})
$$

In particular, $\chi(M)>0$ implies that one of $\chi\left(\mathrm{G} / \mathrm{K}_{ \pm}\right)$is greater than zero, which is equivalent to one of the singular isotropy groups $\mathrm{K}_{ \pm}$having maximal rank in $\mathrm{G}$.

For $G$ simple, the maximal subgroups of maximal rank have been classified by Borel and Siebenthal (see e.g. Goto and Grosshans [1978]), up to an automorphism of $\mathrm{G}$. This can easily be generalized to semisimple and compact groups, as well as to the non-maximal subgroups. We list the result for the classical groups in table 15 on page 65 .

Corollary 1.7. Suppose $\chi(M)>0$ and that $\mathrm{K}_{+}$has the same rank as $\mathrm{G}$. Then the group $\mathrm{H}$ has corank 1 in $\mathrm{G}$, i.e. the sphere $\mathrm{S}^{l_{+}}$is odd dimensional. Proof. Suppose $l_{+}$is even. Suppose $p \in \mathrm{G} / \mathrm{K}_{+}$is in the singular orbit given by $\mathrm{K}_{+}$. Then the dimension of the slice representation at $p$ is given by $\operatorname{dim} M-\operatorname{dim} \mathrm{G} / \mathrm{K}_{+}$. But both $M$ and $\mathrm{G} / \mathrm{K}_{+}$have positive euler characteristic, so they are even dimensional, and so is the slice representation at $p$. But then the sphere of the slice at $p$ is odd dimensional, yet its dimension is $l_{+}$, a contradiction.

We note that in the case that $\operatorname{corank}(\mathrm{H})=1$ and $\operatorname{corank}\left(\mathrm{K}_{+}\right)=0$ we can derive $\chi(M)>0$. This is not true in general for $\operatorname{corank}(\mathrm{H})=0$. 


\subsection{Properties of the isotropy groups}

Again, we carry over the assumptions and notation from 1.1.2 on page 12 and 1.3 on page 14 . Since $G$ is compact, it is finitely covered by a group $\tilde{G}$ of the form $T^{k} \times G_{1} \times \cdots \times G_{l}$ where $T^{k}$ is a $k$-dimensional torus, the center of $\tilde{\mathrm{G}}$, and $\mathrm{G}_{1}, \ldots, \mathrm{G}_{l}$ are simple normal subgroups. We call $k+l$ the number of factors of $G$, which is well-defined since $G$ is a finite quotient of $\tilde{G}$. The fact that if $G$ acts transitively on a sphere, the isotropy group has at least $k+l-1$ factors (see table 16 on page 65 ) leads to the following lemma:

Lemma 1.8. If $\mathrm{G}$ acts almost effectively and primitively, $\mathrm{K}_{+}$has at most 4 factors.

Proof. Denote the number of factors of a group $\mathrm{H}$ by $f(\mathbf{H})$. Since $\mathbf{H}_{ \pm}$are normal subgroups of $\mathrm{H}$, it is clear that

$$
f\left(\mathrm{H}_{+}\right)+f\left(\mathrm{H}_{-}\right)-f\left(\mathrm{H}_{+} \cap \mathrm{H}_{-}\right) \leq f(\mathrm{H})
$$

so that

$$
f\left(\mathrm{H}_{+}\right)+f\left(\mathrm{H}_{-}\right)-f(\mathrm{H}) \leq f\left(\mathrm{H}_{+} \cap \mathrm{H}_{-}\right)
$$

By the classification of effictive transitive actions on spheres (see table 16 on page 65) we have $f(\mathrm{H})-f\left(\mathrm{H}_{ \pm}\right)=f\left(\mathrm{H} / \mathrm{H}_{ \pm}\right) \leq 2$, which implies

$$
2 f(\mathrm{H})-f\left(\mathrm{H}_{-}\right)-f\left(\mathrm{H}_{+}\right) \leq 4
$$

and consequently

$$
f(\mathrm{H})-4 \leq f\left(\mathrm{H}_{+}\right)+f\left(\mathrm{H}_{-}\right)-f(\mathrm{H}) \leq f\left(\mathrm{H}_{+} \cap \mathrm{H}_{-}\right)
$$

But now $f\left(\mathrm{H}_{+} \cap \mathrm{H}_{-}\right)=0$ by lemma 1.3 on page 14 , so that $f(\mathrm{H}) \leq 4$. By the classification of sphere actions we know $f\left(\mathrm{~K}_{+}\right) \leq 5$, but if $f\left(\mathrm{~K}_{+}\right)=5$, we know that $\mathrm{K}_{+} / \mathrm{H}=\mathrm{S}^{1}$ or $\mathrm{K}_{+} / \mathrm{H}=\mathrm{S}^{3}$, and $(\mathrm{H})_{0}=\left(\mathrm{H}_{+}\right)_{0}$ has 4 factors. This is a contradiction to $\mathrm{H}_{+} \subset \mathrm{H} / \mathrm{H}_{-}$, given by primitivity. So $f\left(\mathrm{~K}_{+}\right) \leq 4$.

Remark 1.9. Since we are concerned with almost effective actions, any factor of $\mathrm{K}_{+}$that acts trivially on $\mathrm{K}_{+} / \mathrm{H}$ (that is, is contained in $\mathrm{H}_{+}$), cannot act trivially on $\mathrm{K}_{-} / \mathrm{H}$, so it has to occur as a factor of an isotropy group of an effective transitive sphere action in table 16 on page 65, up to finite quotients. If $\mathrm{K}_{+}$has 4 factors $\mathrm{K}_{1} \cdots \mathrm{K}_{4}$, we can after rearranging the order assume that $\mathrm{K}_{1} \mathrm{~K}_{2}$ act transitively almost effectively on a sphere, and $\mathrm{K}_{3} \mathrm{~K}_{4}$ appear as the isotropy group of a transitive effective action on a sphere, up to finite quotients. This will limit the choices when looking for possibilities for $\mathrm{K}_{+}$from table 15 on page 65 . 
Lemma 1.10. The kernel of the action is the largest normal subgroup shared by $\mathrm{G}$ and $\mathrm{H}$

Proof. It is clear that the kernel of the action is a normal subgroup of both $\mathrm{G}$ and $\mathrm{H}$. Conversely, any subgroup of $\mathrm{H}$ which is normal in $\mathrm{G}$ fixes the whole geodesic $c$ pointwise, and since it's normal, it fixes the whole of $M$.

Remark 1.11. For $\mathrm{G}$ simple, the only normal subgroups are finite and therefore central. So the kernel of the action is the intersection of $\mathrm{H}$ with the center of $\mathrm{G}$.

\subsection{Known classification results}

\subsubsection{Cohomogeneity one manifolds of low dimension}

Cohomogeneity one manifolds of dimension up to seven have been classified, with no assumption on the Euler characteristic: Neuman (Neumann [1968]) classified those of dimension three, Parker (Parker [1986]) those of dimension four (with one omission, as observed by C. Hoelscher), and Hoelscher (Hoelscher [2007]) classified those of dimension five to seven.

\subsubsection{Cohomogeneity one manifolds with a fixed point}

If the action of $\mathrm{G}$ on $M$ has a fixed point, the classification is particularly easy: There are the obvious actions with two fixed points on spheres, and the following groups acting on compact rank one symmetric spaces:

$$
\begin{aligned}
& \mathbb{C P}^{n}: \operatorname{SU}(n), \mathrm{U}(n) \\
& \mathbb{H}^{n}: \operatorname{Sp}(n), \operatorname{Sp}(n) \times \mathrm{Sp}(1), \operatorname{Sp}(n) \times \mathrm{U}(1) \\
& \mathbb{C P}^{2 n+1}: \operatorname{Sp}(n), \operatorname{Sp}(n) \times \mathrm{U}(1) \\
& C a \mathbb{P}^{2}: \operatorname{Spin}(9)
\end{aligned}
$$

The details can be found in Hoelscher [2007] 


\section{Proof of theorem 0.1}

We start with a little lemma concerning orbit equivalent subactions, which will be used in the proof:

Lemma 2.1. Let $M$ be the cohomogeneity one G-manifold given by the diagram $\mathrm{H} \subset \mathrm{K}_{-}, \mathrm{K}_{+}$, and suppose $\mathrm{G}=\mathrm{G}_{1} \times \mathrm{G}_{2}$. If the projection of $\mathrm{H}$ onto the second factor is $\mathrm{G}_{2}$, then the subaction of $\mathrm{G}_{1} \times 1$ on $M$ is also cohomogeneity one.

Proof. The claim be tested on any orbit $\mathrm{G} / \mathrm{G}_{x}$, where $\mathrm{G}_{x}$ is one of $\mathrm{H}, \mathrm{K}_{ \pm}$. For any $\left(g_{1}, g_{2}\right) \mathrm{G}_{x} \in \mathrm{G} / \mathrm{G}_{x}$, there is some $\left(h_{1}, g_{2}\right) \in \mathrm{H}$ by the assumption. Then $\left(g_{1}, g_{2}\right) \mathrm{G}_{x}=\left(g_{1} h_{1}^{-1}, 1\right)\left(h_{1}, g_{2}\right) \mathrm{G}_{x}=\left(g_{1} h_{1}^{-1}, 1\right) \mathrm{G}_{x}$, so $\left(g_{1}, g_{2}\right) \mathrm{G}_{x}$ is in the $\mathrm{G}_{1} \times 1$-orbit of $(1,1) \mathrm{G}_{x}$.

We can now prove theorem 0.1 on page 5 .

Proof. By virtue of $M$ having positive euler characteristic, we can assume $\mathrm{K}_{+}$has maximal rank in $\mathrm{G}$. We also assume $\mathrm{G}=\mathrm{G}_{1} \times \cdots \times \mathrm{G}_{l}$ where each $\mathrm{G}_{i}$ is either simple or $\mathrm{S}^{1}$. Then also $\mathrm{K}_{+}=\mathrm{K}_{+}^{1} \times \cdots \times \mathrm{K}_{+}^{l}$, where each $\mathrm{K}_{+}^{i}$ is a subgroup of $\mathrm{G}_{i}$ of maximal rank, and we can assume that $\mathrm{K}_{+}^{1}$ acts transitively on the sphere $\mathrm{K}_{+} / \mathrm{H}$. Let $p r_{i}$ be the projection from $\mathrm{G}$ onto the $i$-th factor. By the lemma above, $\operatorname{pr}_{i}(\mathrm{H}) \neq \mathrm{G}_{i}$ for all $i=1, \ldots, l$.

We claim $l=2$. Suppose $l>2$ and let $p_{2}: \mathrm{G} \rightarrow \mathrm{G}_{2} \times \cdots \times \mathrm{G}_{l}$ be the projection. Because $\mathrm{K}_{+}^{1}$ acts transitively, we have $p_{2}\left(\mathrm{~K}_{+}\right)=p_{2}(\mathrm{H})$, and primitivity implies $p_{2}\left(\mathrm{~K}_{-}\right)=\mathrm{G}_{2} \times \cdots \times \mathrm{G}_{l}$ (otherwise, $\mathrm{K}_{ \pm} \subset p_{2}^{-1}\left(p_{2}\left(\mathrm{~K}_{-}\right)\right) \neq \mathrm{G}$, a contradiction to primitivity). But $p r_{2}(\mathrm{H}) \neq \mathrm{G}_{2}$, so $p_{2}^{-1}\left(\mathrm{G}_{2}\right) \cap \mathrm{K}_{-}$acts transitively on $\mathrm{K}_{-} / \mathrm{H}$, which implies $p_{3}\left(\mathrm{~K}_{-}\right)=p_{3}(\mathrm{H})$ for the projection $p_{3}$ : $\mathrm{G} \rightarrow \mathrm{G}_{3} \times \cdots \times \mathrm{G}_{l}$. By the lemma above, $\mathrm{G}_{1} \times \mathrm{G}_{2}$ acts orbit equivalent, a contradiction. Therefore $l=2$.

For now suppose that $\mathrm{K}_{-} \cap \mathrm{G}_{2}$ is not finite. We have $p r_{2}\left(\mathrm{~K}_{-}\right)=\mathrm{G}_{2}$, so $\mathrm{K}_{-} \cap \mathrm{G}_{2}$ is a normal subgroup of $\mathrm{G}_{2}$ (if $\left(1, k_{2}\right) \in \mathrm{K}_{-} \cap \mathrm{G}_{2}$ and $g_{2} \in \mathrm{G}_{2}$ is arbitrary, then there is some $\left(k, g_{2}\right) \in \mathrm{K}_{-}$, and $\left(1, g_{2}\right)\left(1, k_{2}\right)\left(1, g_{2}^{-1}\right)=$ $\left.\left(k, g_{2}\right)\left(1, k_{2}\right)\left(k^{-1}, g_{2}^{-1}\right) \in \mathrm{K}_{-} \cap \mathrm{G}_{2}\right)$. By assumption, $\mathrm{K}_{-} \cap \mathrm{G}_{2}=\mathrm{G}_{2}$. But $p r_{2}(\mathrm{H}) \neq \mathrm{G}_{2}$, so $\mathrm{G}_{2} \subset \mathrm{K}_{-}$acts transitively on $\mathrm{K}_{-} / \mathrm{H}$, and therefore $p r_{1}\left(\mathrm{~K}_{-}\right)=$ $p r_{1}(\mathrm{H})$. By primitivity, $p r_{1}\left(\mathrm{~K}_{+}\right)=\mathrm{G}_{1}$, and from $\mathrm{K}_{+}$having the same rank as $G$ we can deduce that $G_{1}$ is a normal subgroup of $K_{+}$. We now divide cases by the dimension of the sphere $S^{l_{-}}$:

- Suppose $l_{-}$is even, that is, the rank of $\mathrm{K}_{-}$is the same as the rank of $\mathrm{H}$. That implies that $p r_{2}(\mathrm{H})$ has full rank in $\mathrm{G}_{2}$ and therefore $\mathrm{H}$ is a product subgroup of $\mathrm{G}$. So we have 


$$
\begin{aligned}
\mathrm{K}_{-} & =\mathrm{K}_{-}^{1} \times \mathrm{G}_{2} \\
\mathrm{H} & =\mathrm{K}_{-}^{1} \times \mathrm{H}_{2} \\
\mathrm{~K}_{+} & =\mathrm{G}_{1} \times \mathrm{H}_{2}
\end{aligned}
$$

where $G_{1} / K_{-}^{1}$ and $G_{2} / H_{2}$ are spheres. This is easily recognized as a so called sum-action on a sphere of even dimension (see e.g. Hoelscher [2007]).

- If $l_{-}$is odd, that is, the $\operatorname{rank}$ of $\mathrm{K}_{-}$is $\operatorname{rank}(\mathrm{H})+1=\operatorname{rank}(\mathrm{G})$, then we have the following situation: $\mathrm{K}_{-}=\mathrm{K}_{-}^{1} \times \mathrm{G}_{2}, \mathrm{~K}_{+}=\mathrm{G}_{1} \times \mathrm{K}_{+}^{2}$. If we define $\mathrm{H}_{i}:=\mathrm{H} \cap \mathrm{G}_{i}$, we have that $\mathrm{G}_{i} / \mathrm{H}_{i}$ is a sphere for $i=1,2$, and $\mathrm{H}_{1} \times \mathrm{H}_{2}$ is a normal subgroup of $\mathrm{H}$ of corank 1 . Then we can find a rank 1 normal subgroup $\Delta \mathrm{H}$ of $\mathrm{H}$ that commutes with $\mathrm{H}_{1} \times \mathrm{H}_{2}$ such that $\mathrm{H}=\left(\mathrm{H}_{1} \times \mathrm{H}_{2}\right) \Delta \mathrm{H}$. We have $\mathrm{K}_{-}^{1}=p r_{1}(\mathrm{H})$ and $\mathrm{K}_{+}^{2}=p r_{2}(\mathrm{H})$.

Now consider the cohomogeneity one $\mathrm{G}_{1} \times \mathrm{G}_{2}$-manifold given by the group diagram $\mathrm{H}_{1} \times \mathrm{H}_{2} \subset \mathrm{H}_{1} \times \mathrm{G}_{2}, \mathrm{G}_{1} \times \mathrm{H}_{2}$, which is a sphere $\mathrm{S}^{2 n+1}$ of odd dimension as above. We claim that $M$ is the quotien of $\mathrm{S}^{2 n+1}$ by a free action of $\mathrm{H} /\left(\mathrm{H}_{1} \times \mathrm{H}_{2}\right)$. For that we only need to consider the following actions of $\mathrm{H} /\left(\mathrm{H}_{1} \times \mathrm{H}_{2}\right)$ on the orbits of $\mathrm{S}^{2 n+1}$ :

- $\mathrm{H} /\left(\mathrm{H}_{1} \times \mathrm{H}_{2}\right)$ acts freely on $\mathrm{G}_{1} / \mathrm{H}_{2} \times \mathrm{G}_{2} / \mathrm{H}_{1}$ with quotient $\mathrm{G}_{1} \times \mathrm{G}_{2} / \mathrm{H}$.

$-\mathrm{H} /\left(\mathrm{H}_{1} \times \mathrm{H}_{2}\right)$ acts freely on $\mathrm{G}_{1} / \mathrm{G}_{1} \times \mathrm{G}_{2} / \mathrm{H}_{2}=\mathrm{G}_{2} / \mathrm{H}_{2}$ with quotient $\mathrm{G}_{2} / p r_{2}(\mathrm{H})$.

- $\mathrm{H} /\left(\mathrm{H}_{1} \times \mathrm{H}_{2}\right)$ acts freely on $\mathrm{G}_{1} / \mathrm{H}_{1} \times \mathrm{G}_{2} / \mathrm{G}_{2}=\mathrm{G}_{1} / \mathrm{H}_{1}$ with quotient $\mathrm{G}_{1} / \operatorname{pr}_{1}(\mathrm{H})$.

In conclusion, the orbits of the action of $G$ on the quotient of $S^{2 n+1}$ by $\mathrm{H} /\left(\mathrm{H}_{1} \times \mathrm{H}_{2}\right)$ are those of the action of $\mathrm{G}$ on $M$, which finishes this part of the proof (see section 1 on page 12).

Lastly, consider $\mathrm{K}_{-} \cap \mathrm{G}_{2}$ finite. Then $\operatorname{rank}\left(\mathrm{G}_{2}\right)=1$ and $\mathrm{K}_{-} / \mathrm{H}$ is even dimensional (if $\operatorname{rank}\left(\mathrm{K}_{-}\right)=\operatorname{rank}(\mathrm{G})$, i.e. the sphere is odd dimensional, then $\mathrm{K}_{-}$is a product with $p r_{2}\left(\mathrm{~K}_{-}\right)=\mathrm{G}_{2}$, a contradiction; also $\mathrm{K}_{-} \cap \mathrm{G}_{2}$ has corank 0 or 1 in $G_{2}$, which implies $\operatorname{rank}\left(G_{2}\right) \leq 1$ ). We also have $p r_{2}\left(\mathrm{~K}_{-}\right)=\mathrm{G}_{2}$ and $p r_{2}(\mathrm{H}) \neq \mathrm{G}_{2}$, so $\mathrm{G}_{2}$ is covered by $\mathrm{S}^{3}$ and $\mathrm{K}_{-} / \mathrm{H}=\mathrm{S}^{2}$. 


\section{$3 \quad$ The general procedure}

In this section we will describe the actual procedure for the classification. We will treat each of the simple groups separately, and make extra sections for $\mathrm{SU}(3)$ and $\mathrm{SU}(4)$.

For each classical simple group $\mathrm{G}$, we will first list the result, the table of group diagrams of cohomogeneity one G-manifolds up to equivalence. In order to prove this result, we will then list the possibilities for the subgroup $\mathrm{K}_{+}$of maximal rank, combining table 15 on page 65 , lemma 1.8 on page 16 and remark 1.9 on page 16. This is simply an exercise in book-keeping. The conditions listed ensure that the different cases really are disjoint. Note that for the sake of organisation we divided cases such as $\mathrm{SO}(n), n \geq 2$ into the cases $\mathrm{SO}(2)$ and $\mathrm{SO}(n), n \geq 3$.

By what was said in section 1.2 on page 13 , we can assume $\mathrm{K}_{+}$has the standard block structure. We will then use table 16 on page 65 to list the possibilities for the isotropy group $\mathrm{H}$ of the action of $\mathrm{K}_{+}$on the sphere $\mathrm{K}_{+} / \mathrm{H}$. Again, wen can conjugate the diagram by an element of $\mathrm{K}_{+}$to ensure $\mathrm{H}$ is of a given form. After that, we can again use the same table to list the possibilities for $\mathrm{K}_{-}$. Lemma 1.3 on page 14 will be used without further mention to discard some of the possibilities. The last step is to check the possible embeddings of $K_{-}$into $G$, i.e. which are equivalent and which give a primitive diagram.

\subsection{The Spin-groups}

The cases of the Spin-groups will be divided into two different cases each. First, we will classify the non-effective actions, i.e. those that are actually action of the special orthogonal group. After that, we will classify the effective actions of the Spin-groups. The procedure for the latter ones differs slightly from the general procedure described above: We will apply the projection $\pi: \operatorname{Spin}(n) \rightarrow \mathrm{SO}(n)$ to the whole diagram and classify the resulting diagrams. The list of subgroups of maximal rank is easily deduced from table 15 on page 65 .

Since we know the action is effective, we have $-1 \notin \mathrm{H}$ (where -1 is the element that projects to the identity of $\mathrm{SO}(n)$ but which is not the identity element of $\operatorname{Spin}(n))$ by remark 1.11 on page 17 . This shortens the list of possibilities for $\mathrm{K}_{+}$, because it implies that $\mathrm{H}$ does not contain a subgroup of type $\operatorname{Spin}(n), n \geq 3$.

We have $-1 \in \mathrm{K}_{+}$from the following fact: The preimage $\pi^{-1}(\mathrm{~K})$ of a subgroup $\mathrm{K} \subset \mathrm{SO}(n)$ is connected if and only if the inclusion $\mathrm{K} \hookrightarrow \mathrm{SO}(n)$ induces a surjection on the fundamental group. This is the case for all max- 
imal rank subgroups of $\mathrm{SO}(n)$, so their preimages contain -1 in the unity component. This implies that $\pi\left(\mathrm{K}_{+}\right) / \pi(\mathrm{H})$ is a real projective space, and the possibilities for $\pi(\mathrm{H})$ can be deduced from table 16 on page 65. A priori, we cannot assume $-1 \in \mathrm{K}_{-}$, so we list the possibilities for $\pi\left(\mathrm{K}_{-}\right)$under the assumption that $\pi\left(\mathrm{K}_{-}\right) / \pi(\mathrm{H})$ is either a sphere or a projective space. For a subgroup of $\mathrm{SO}(n)$ of a given isomorphism type it is easy to decide whether the above criterion applies, so we can decide if $\mathrm{K}_{-} / \mathrm{H}$ is a sphere, and carry on as above.

We will find that $-1 \in \mathrm{K}_{\text {- }}$ in each case. The diagram can then be reconstructed by the fact that then $\mathrm{K}_{ \pm}=\pi^{-1}\left(\pi\left(\mathrm{K}_{ \pm}\right)\right)$and $\mathrm{H}=\pi^{-1}(\pi(\mathrm{H}))_{0}$. For convenience of notation, we will always give the $\mathrm{SO}(n)$-diagram $\pi(\mathrm{H}) \subset$ $\pi\left(\mathrm{K}_{-}\right), \pi\left(\mathrm{K}_{+}\right)$. By abuse of notation, we will discard the $\pi$, which will not lead to confusion, since everything is discussed in $\mathrm{SO}(n)$ anyways.

There is another simple fact we will make use of. In the situation described above, note that $\mathrm{SO}(n) / \pi(\mathrm{H})$ is not simply connected, because $\operatorname{Spin}(n) / \mathrm{H}$ is a nontrivial cover. This implies in particular that $\pi(\mathrm{H}) \hookrightarrow \mathrm{SO}(n)$ does not induce a surjection on the fundamental group.

\subsubsection{Notation}

We adopt the following notation: The defining representations of $\mathrm{SO}(n)$, $\mathrm{SU}(n)$, and $\operatorname{Sp}(n)$ will be denoted by $\rho_{n}, \mu_{n}$, and $\nu_{n}$ respectively. We will use $\rho_{o}$ to denote the trivial one-dimensional representation of any group.

We assume that a fundamental system of roots has been chosen for each of the simple groups. Any irreducible representation of a simple lie group (of rank $n$ ) is given by a string of $n$ nonnegative integers, which gives the heighest weight of the representation as a linear combination of the fundamental roots. We will denote this representation by its string $\left(c_{1}, \ldots, c_{n}\right)$. So e.g. $(1,0)$ will denote the first fundamental representation of a rank 2 simple lie group. By the dimension formula of Weyl it is clear that the dimension of an irreducible representation of a simple lie group is strictly increasing in the entries of the string in the following sense: If $\left(c_{1}, \ldots, c_{n}\right)$ and $\left(c_{1}^{\prime}, \ldots, c_{n}^{\prime}\right)$ denote are two irreducible representations of the same simple lie group, and we have $c_{i} \leq c_{i}^{\prime}$, then $\operatorname{dim}\left(c_{1}, \ldots, c_{n}\right) \leq \operatorname{dim}\left(c_{1}^{\prime}, \ldots, c_{n}^{\prime}\right)$. If additionally $c_{j}$ is strictly smaller than $c_{j}^{\prime}$ for at least one index $1 \leq j \leq n$, then the inequality is strict.

We will denote the lie algebras of the groups $\mathrm{G}, \mathrm{K}_{-}, \mathrm{K}_{+}, \mathrm{H}, \mathrm{H}_{-}$and $\mathrm{H}_{+}$ by $\mathfrak{g}, \mathfrak{k}_{-}, \mathfrak{k}_{+}, \mathfrak{h}, \mathfrak{h}_{-}$and $\mathfrak{k}_{+}$, as usual. 


\section{$4 \quad \mathrm{G}=\mathrm{SU}(3)$}

For a general overview of the classification procedure, see section 3 on page 20 . We claim that up to equivalence, the diagrams of the simply connected primitive cohomogeneity one SU(3)-manifolds with positive euler characteristic are given by table 1 .

Table 1: SU(3)-cohomogeneity one manifolds

\begin{tabular}{|l|}
\hline $\mathrm{S}^{1} \subset \mathrm{SU}(2), \mathrm{U}(2)$ \\
\hline $\mathrm{S}^{1} \subset \mathrm{S}(\mathrm{U}(2) \mathrm{U}(1)), \mathrm{S}(\mathrm{U}(1) \mathrm{U}(2))$ \\
\hline $\mathrm{S}^{1} \subset \mathrm{SO}(3), \mathrm{S}(\mathrm{U}(1) \mathrm{U}(2))$ \\
\hline $\mathrm{S}^{1} \subset \mathrm{SO}(3), \mathrm{T}^{2}$ \\
\hline $\mathbb{Z}_{3} \mathrm{SO}(3) \subset \mathbb{Z}_{3} \mathrm{SO}(3), \mathrm{T}^{2}$ \\
\hline
\end{tabular}

By the classification of Borel an Siebenthal (see table 15 on page 65 ), we have $\mathrm{K}_{+}=\mathrm{U}(2)$ or $\mathrm{K}_{+}=\mathrm{T}^{2}$. If $\operatorname{dim} \mathrm{H} \geq 2$, we have $\operatorname{dim} M=\operatorname{dim} \mathrm{G} / \mathrm{H}+1 \leq$ 7, so $M$ appears in Hoelscher [2007]. So we will assume $\mathrm{H}=\mathrm{S}^{1}$ for the rest of this section.

First assume $\mathrm{K}_{+}=\mathrm{U}(2)$, where $\mathrm{SU}(2)$ is embedded in the lower right block. By what was said above and what follows from B.2 on page 66 , we have $\mathrm{H}=\mathrm{S}_{k}^{1}$ where

$$
\mathrm{S}_{k}^{1}=\left\{\operatorname{diag}\left(\bar{z}, z^{k+1}, z^{-k}\right) \mid z \in \mathrm{S}^{1}\right\}
$$

If $\mathrm{K}_{-}$acts almost effectively $\mathrm{S}^{l_{-}}$, we have $\mathrm{K}_{-} \in\{\mathrm{U}(2), \mathrm{SO}(3), \mathrm{SU}(2)\}$. Otherwise $\mathrm{K}_{-}=\mathrm{T}^{2}$, which we will treat later in this section, or $\mathrm{K}_{-}=\mathrm{S}_{k}^{1} \mathrm{SU}(2)$ where $S_{k}^{1}$ is normal in $K_{-}$and does not intersect $S U(2)$. The first is only possible for $k=-2$ or $k=1$, but in both cases the (unique) $\mathrm{SU}(2)$ in its normalizer is intersected.

For an almost effective action of $\mathrm{SU}(2)$, we need to find an $\mathrm{SU}(2)$ that contains $\mathrm{S}_{k}^{1}$, which implies $k=-1$ or $k=0$. Both cases are equivalent by a change of the last 2 coordinates, which fixes $\mathrm{K}_{+}$, so we can assume $k=0$, which gives a primitive example, because $N(\mathrm{H})_{0}=\mathrm{T}^{2}$. If $\mathrm{K}_{-} \simeq \mathrm{U}(2)$, we will argue that we can assume both $\mathrm{K}_{ \pm}$contain the same maximal torus. Of course, we can conjugate the maximal torus of $\mathrm{K}_{-}$into the standard one, and by changing the conjugation with an element of $\mathrm{K}_{+}$, we may assume it preserves $S_{k}^{1}$. But then both $S_{k}^{1}$ and its conjugate are diagonal, so by changing 
the conjugation with an element of the Weyl group, we may assume it actually preserves $\mathrm{S}_{k}^{1}$ pointwise, so it is contained in its centralizer, which is in $N\left(\mathrm{~S}_{k}^{1}\right)_{0}$. Now both $\mathrm{K}_{ \pm}$contain the same maximal torus, so they are conjugate by an element of the Weyl group, and since one of those elements fixes $\mathrm{K}_{+}$, we can assume $\mathrm{K}_{-}=\mathrm{S}(\mathrm{U}(2) \mathrm{U}(1))$. Checking the possible isotropy groups of $\mathrm{K}_{-}$, we see $k= \pm 1$. For $k=1$, the normalizer of $\mathrm{S}_{1}^{1}$ contains an $\mathrm{SU}(2)$ in which we can realize the exchange of the first and the third coordinate, transforming $\mathrm{K}_{-}$into $\mathrm{K}_{+}$, so this is not a primitive example. For $k=-1$, we have $N\left(S_{-1}^{1}\right)_{0}=\mathrm{T}^{2}$, so this example is primitive.

Now we will show that there is one example for $\mathrm{K}_{-}=\mathrm{SO}(3)$. For that we will argue that $\mathrm{K}_{-}$is conjugate to the standard $\mathrm{SO}(3)$ in $N\left(\mathrm{~S}_{k}^{1}\right)_{0}$. Since there is only one 3 -dimensional representation of $\mathrm{SO}(3)$, we know $\mathrm{K}_{-}$is conjugate to the standard subgroup. But all $\mathrm{SO}(2) \subset \mathrm{SO}(3)$ are conjugate, so we may assume this conjugation preserves the standard $\mathrm{SO}(2)$, i.e. it is in its normalizer $\mathrm{O}(2) \times \mathrm{S}^{1}$, where $\mathrm{S}^{1}$ is given by $\operatorname{diag}\left(z, z, \bar{z}^{2}\right)$. But every conjugation of $\mathrm{O}(2)$ on $\mathrm{SO}(2)$ can be realized in $\mathrm{SO}(2)$ itself, so we can assume the conjugation comes from $N(\mathrm{SO}(2))_{0}$. But now $\mathrm{S}_{k}^{1}$ can only be contained in $\mathrm{SO}(3)$ if it's conjugate to $\mathrm{SO}(2)$, so the embedding $\mathrm{S}_{k}^{1} \hookrightarrow \mathrm{SU}(3)$ must have a 1-dimensional trivial subrepresentation, implying $k=0,-1$. Both of those are conjugate to the standard $\mathrm{SO}(2)$, so by the argument above we can assume $K_{-}$is the standard $S_{0}(3)$. Since $S_{-1}^{1}$ and $S_{0}^{1}$ can be transformed into each other by an outer automorphism of $\mathrm{K}_{+}$(complex conjugation, which is also an automorphism of $\mathrm{SU}(3)$ ) that leaves $\mathrm{SO}(3)$ invariant, we just get 1 example from this case.

The second and last case to consider is $\mathrm{K}_{+}=\mathrm{T}^{2}$. We have $\mathrm{H}_{0}=\mathrm{S}^{1}$, therefore $\left(\mathrm{K}_{-}\right)_{0}$ is one of $\mathrm{SO}(3), \mathrm{U}(2), \mathrm{SU}(2), \mathrm{T}^{2}$.

If $\left(\mathrm{K}_{-}\right)_{0}=\mathrm{T}^{2}$, it is contained in the centralizer of $\mathrm{H}_{0}$ as well as $\left(\mathrm{K}_{+}\right)_{0}$, so both are conjugate in $N(\mathrm{H})_{0}$ in particular. Since a maximal torus has finite index in its normalizer, this shows that no primitive example arises in this case.

If $\left(\mathbf{K}_{-}\right)_{0}=\mathbf{S U}(2)$, then $\mathrm{H}_{0}$ is a maximal torus in $\mathbf{K}_{-}$. By conjugating the diagram we may assume $\left(\mathrm{K}_{-}\right)_{0}$ is given by the lower $2 \mathrm{x} 2$-block, and $\mathrm{H}_{0}$ the standard maximal torus therein. This determines the maximal torus $\mathrm{T}^{2}$ in $\mathrm{SU}(3)$, and both of $\mathrm{K}_{-}$and $\mathrm{K}_{+}$are contained in $\mathrm{U}(2)$.

For $\left(\mathrm{K}_{-}\right)_{0}=\mathrm{U}(2)$ : If $\mathrm{H}_{0}$ is regular, i.e. $Z(\mathrm{H})_{0}=\mathrm{T}^{2} \subset \mathrm{U}(2)$, this is obviously not primitive. If $\mathrm{H}_{0}$ is not regular, its isotropy representation has 2 equal eigenvalues, and we may assume we have $\mathfrak{h}=\mathbb{R} \cdot \operatorname{diag}(i, i,-2 i)$. Its isotropy representation therefore has 2 equivalent 2-dimensional factors, which are in fact equivalent in $N(\mathrm{H})_{0}$, so we can conjugate $\left(\mathrm{K}_{-}\right)_{0}$ into 


$$
\mathrm{U}(2)=\left\{\left(\begin{array}{cc}
\operatorname{det} \bar{A} & 0 \\
0 & A
\end{array}\right) \mid A \in \mathrm{U}(2)\right\}
$$

without changing $\mathrm{T}^{2}$, so there is also no new primitive example.

In the case $\left(\mathrm{K}_{-}\right)_{0}=\mathrm{SO}(3), \mathrm{K}_{-}$is actually given by the standard embedding, for $\mathrm{SO}(3)$ has no outer automorphisms and its only faithfull 3dimensional representation is irreducible. The latter also implies that its centralizer is given by $\mathbb{Z}_{3}$, the set of diagonal matrices, so we have $N(\mathrm{SO}(3))=$ $\mathrm{SO}(3) \mathbb{Z}_{3}$. Since $T^{2}$ is uniquely determined by $\mathrm{H}_{0}=\mathrm{S}^{1}$, we obtain two new examples, both of which are primitive for the isotropy representation of $\mathrm{SO}(3)$ in $\mathrm{SU}(3)$ is irreducible, so $\mathrm{SO}(3)$ is a maximal subgroup not containing $\mathrm{K}_{+}$. The examples are $\mathrm{S}^{1} \subset\left\{\mathrm{SO}(3), \mathrm{T}^{2}\right\}$ and $\mathrm{S}^{1} \mathbb{Z}_{3} \subset\left\{\mathrm{SO}(3) \mathbb{Z}_{3}, \mathrm{~T}^{2}\right\}$. Note that $\mathrm{K}_{+}$is connected, since $l_{-}=2$. 


\section{$5 \quad \mathrm{G}=\mathrm{SU}(4)$}

For a general overview of the classification procedure, see section 3 on page 20 . For special remarks regarding Spin-groups (we have SU(4) $\simeq \operatorname{Spin}(6)$ ), see section 3.1 on page 20 .

We claim that up to equivalence the diagrams of the simply connected primitive cohomogeneity one SU(4)-manifolds with positive euler characteristic are given by tables 2 and 3 .

Table 2: SO(6)-cohomogeneity one manifolds

\begin{tabular}{|c|}
\hline $\mathrm{SO}(4) \subset \mathrm{SO}(5), \mathrm{SO}(2) \mathrm{SO}(4)$ \\
\hline $\mathbb{Z}_{2} \mathrm{SO}(4) \subset \mathbb{Z}_{2} \mathrm{SO}(5), \mathrm{SO}(2) \mathrm{SO}(4)$ \\
\hline $\mathrm{SO}(2) \mathrm{SO}(3) \subset \mathrm{SO}(3) \mathrm{SO}(3), \mathrm{SO}(2) \mathrm{SO}(4)$ \\
\hline $\mathrm{SO}(2) \mathrm{SO}(2) \subset \mathrm{SO}(2) \mathrm{SO}(3), \mathrm{U}(2) \mathrm{SO}(2)$ \\
\hline $\mathrm{U}(2) \subset \mathrm{SO}(4), \mathrm{U}(3)$ \\
\hline $\mathrm{T}^{2} \subset \mathrm{SO}(3) \mathrm{SO}(2), \mathrm{SO}(2) \mathrm{U}(2)$ \\
where $\mathrm{T}^{2}=\left\{\operatorname{diag}\left(z_{1}, 1,1, z_{2}\right)\right\}$ \\
\hline
\end{tabular}

Table 3: SU(4)-cohomogeneity one manifolds

\begin{tabular}{|c|}
\hline $\begin{array}{c}\mathrm{S}^{1} \mathrm{SU}(2) \subset \mathrm{S}(\mathrm{U}(2) \mathrm{U}(2)), \mathrm{S}(\mathrm{U}(1) \mathrm{U}(3)) \\
\text { where } \mathrm{S}^{1}=\left\{\operatorname{diag}\left(\bar{z}^{2}, z^{4}, \bar{z}, \bar{z}\right)\right\} \subset N(\mathrm{SU}(2))\end{array}$ \\
\hline $\begin{array}{c}\mathrm{S}^{1} \mathrm{SU}(2) \subset \mathrm{S}(\mathrm{U}(2) \mathrm{U}(2)), \mathrm{S}(\mathrm{U}(1) \mathrm{U}(3)) \\
\text { where } \mathrm{S}^{1}=\left\{\operatorname{diag}\left(\bar{z}^{2}, 1, z, z\right)\right\} \subset N(\mathrm{SU}(2))\end{array}$ \\
\hline $\mathrm{S}^{1} \subset \sigma(\mathrm{S}(\mathrm{U}(1) \mathrm{U}(3))), \mathrm{S}(\mathrm{U}(1) \mathrm{U}(3))$ \\
where $\mathrm{S}^{1}=\{\operatorname{diag}(\bar{z}, z, 1,1)\}$ and $\sigma$ exchanges the first two coordinates \\
\hline
\end{tabular}

By the classification of Borel and Siebenthal, given in table 15 on page 65 and remark 1.9 on page 16 , we know $\mathrm{K}_{+}$is one of $\mathrm{U}(3), \mathrm{S}^{1} \mathrm{SU}(2) \mathrm{SU}(2), \mathrm{S}^{1} \mathrm{U}(2)$.

First, we deal with that case that $\mathrm{Sp}(2)$ is contained in any of the regular isotropy groups. Since $\operatorname{rank}(\mathrm{Sp}(2))=2<\operatorname{rank}(\mathrm{SU}(4))$, and $\mathrm{Sp}(2)$ is a maximal connected subgroup of SU(4), we can deduce $\left(\mathrm{K}_{-}\right)_{0}=\mathrm{Sp}(2)$. Because 
$\mathrm{K}_{+}$has maximal rank in $\mathrm{SU}(4)$, and the rank of $\mathrm{K}_{+}$and $\mathrm{H}$ can differ by at most 1, we see $\operatorname{rank}(\mathrm{H})=\operatorname{rank}\left(\mathrm{K}_{-}\right)$, so $\mathrm{K}_{-} / \mathrm{H}$ must be an even dimensional sphere. By the classification of transitive effective actions on spheres (see table 16 on page 65 ), we know $\mathrm{H}=\mathrm{Sp}(1) \mathrm{Sp}(1)$, where the common central element of $\mathrm{H}, \mathrm{K}_{-}, \mathrm{SU}(4)$ is in the kernel of the action of $\mathrm{G}$, so this is actually an action of $\mathrm{SO}(6)=\mathrm{SU}(4) /\{ \pm I d\}$, and $\left(\mathrm{K}_{-}\right)_{0}=\mathrm{SO}(5),(\mathrm{H})_{0}=\mathrm{SO}(4)$. Since $\operatorname{rank}\left(\mathrm{K}_{+}\right)=3$, we have $\mathrm{K}_{+}=\mathrm{S}^{1} \mathrm{SO}(4)$. Both $\mathrm{K}_{+}$and $\mathrm{H}$ can have at most 2 components, so $S^{1}$ can act with weight 1 or 2 on the slice, giving two primitive examples.

Now we divide cases by $\mathrm{K}_{+}$, under the assumption that $\mathrm{K}_{-}$does not contain $\mathrm{Sp}(2)$ as a factor (which is true for $\mathrm{K}_{+}$by the classification anyways).

So now assume $\mathrm{K}_{+}=\mathrm{U}(3)$, where $\mathrm{SU}(3)$ is the lower right block. If $\mathrm{SU}(3) \subset \mathrm{H}$, then it's easily seen that $\mathrm{K}_{-}=\mathrm{SU}(4)$ by table 16 on page 65 , which is listed in subsection 1.5.2 on page 17. So we can assume $\mathrm{SU}(3) \not \subset$ $\mathrm{H}$, which implies that $\mathrm{U}(3)$ acts almost effectively on the slice, giving $\mathrm{H}=$ $\mathrm{S}_{k}^{1} \mathrm{SU}(2)$, where

$$
\mathrm{S}_{k}^{1}=\left\{\operatorname{diag}\left(\bar{z}^{2}, z^{2(k+1)}, \bar{z}^{k}, \bar{z}^{k}\right) \mid z \in \mathrm{S}^{1}\right\}
$$

and $\mathrm{SU}(2)$ is the lower right block (see section B.2 on page 66). We now divide cases by the rank of $\mathrm{K}_{-}$and its dimension:

- If $\operatorname{rank}\left(\mathrm{K}_{-}\right)=\operatorname{rank}(\mathrm{H})$, we have $\mathrm{K}_{-} / \mathrm{H}=\mathbb{S}^{2}$ and $\mathrm{K}_{-} / \mathrm{H}_{-}=\mathrm{SO}(3)$. Moreover, the semisimple part of $\mathrm{H}$ is contained in $\mathrm{H}_{-}$, and therefore $\mathrm{K}_{-} \subset N(\mathrm{SU}(2))=\mathrm{S}^{1} \mathrm{SU}(2) \mathrm{SU}(2)$. That implies $\mathrm{K}_{-}=\mathrm{SU}(2) \mathrm{SU}(2)$, and since $\mathrm{H} \subset \mathrm{SU}(2) \mathrm{SU}(2)$, we have $k=0$. This leaves one primitive example (note that this action is not effective).

From now on, we can assume $\operatorname{rank}\left(\mathrm{K}_{-}\right)>\operatorname{rank}(\mathrm{H})$. We divide cases by the dimension of $\mathrm{K}_{-} / \mathrm{H}$, which is easily seen to be bounded by 5 .

- If $\mathrm{K}_{-} / \mathrm{H}=\mathbb{S}^{1}$, we have $\mathrm{T}^{2} \mathrm{SU}(2)=\mathrm{K}_{-} \subset N(\mathrm{H})_{0}$. If $k \neq 2$, we have $N(\mathrm{H})_{0}=\mathrm{T}^{2} \mathrm{SU}(2) \subset \mathrm{K}_{+}$, so there will be no primitive example. For $k=-2$, we have $N(\mathrm{H})_{0}=\mathrm{S}^{1} \mathrm{SU}(2) \mathrm{SU}(2)$, and it is easily seen that up to conjugation in $N(\mathrm{H})_{0}$ we have $\mathrm{K}_{-} \subset \mathrm{K}_{+}$. As before, this contradicts primitivity.

- If $\mathrm{K}_{-} / \mathrm{H}=\mathbb{S}^{3}$, then $\mathrm{K}_{-}=\mathrm{S}(\mathrm{U}(2) \mathrm{U}(2))$. Since $\mathrm{K}_{-} / \mathrm{H}$ is a sphere, we have that $\mathrm{SU}(2) \cap \mathrm{H}$ is trivial, where $\mathrm{SU}(2)$ is the upper left block. This leads to $k= \pm 1$ and gives 2 examples, which are obviously primitive.

- Lastly, consider $\mathrm{K}_{-} / \mathrm{H}=\mathbb{S}^{5}$. This implies $\mathrm{K}_{-}=\mathrm{U}(3)$, and by studying the isotropy representation of $\mathrm{SU}(4) / \mathrm{H}$, there are two possibilities for 
$\mathrm{K}_{-}$, but since primitivity implies $\mathrm{K}_{-} \neq \mathrm{K}_{+}$, we know $\mathrm{K}_{-}=\sigma \mathrm{K}_{+} \sigma$, where

$$
\sigma=\left(\begin{array}{cccc}
0 & 1 & 0 & 0 \\
-1 & 0 & 0 & 0 \\
0 & 0 & 1 & 0 \\
0 & 0 & 0 & 1
\end{array}\right)
$$

Then $\mathrm{K}_{-} / \mathrm{H}$ is a sphere if and only if $\sigma \mathrm{SU}(3) \sigma^{-1} \cap \mathrm{H}=\mathrm{SU}(2)$ (where $\mathrm{SU}(3)$ and $\mathrm{SU}(2)$ are the lower right blocks). We easily see that

$$
\sigma \mathrm{SU}(3) \sigma^{-1} \cap \mathrm{H}=\left\{\operatorname{diag}\left(\bar{z}^{2}, z, \bar{z}^{k}, \bar{z}^{k}\right) \cdot A \mid z^{2(k+1)}=1, A \in \mathrm{SU}(2)\right\}
$$

and this implies $k=0,-2$. If $k=0$, we have $N(\mathrm{H})_{0}=\mathrm{T}^{2} \mathrm{SU}(2)$, which does not contain $\sigma$, so this is a primitive example. If $k=-2$, we have $N(\mathrm{H})_{0}=\mathrm{S}^{1} \mathrm{SU}(2) \mathrm{SU}(2)$, so up to conjugation in $N(\mathrm{H})_{0}$ we have $\mathrm{K}_{-}=\mathrm{K}_{+}$, which is not possible. This finishes the case $\mathrm{K}_{+}=\mathrm{U}(3)$.

The cases left are $\left(\mathrm{K}_{+}\right)_{0}=\mathrm{S}(\mathrm{U}(1) \mathrm{U}(1) \mathrm{U}(2))$ and $\left(\mathrm{K}_{+}\right)_{0}=\mathrm{S}(\mathrm{U}(2) \mathrm{U}(2))$. Common for both consider $\mathrm{SU}(2) \subset \mathrm{H}_{+}$. This would imply that $\mathrm{SU}(2)$ needs to act on $\mathrm{K}_{-} / \mathrm{H}$, and therefore $\mathrm{K}_{-}=\mathrm{U}(3)$ or $\mathrm{K}_{-}=\mathrm{Sp}(2)$, which are cases we have considered before.

Now assume $\left(\mathrm{K}_{+}\right)_{0}=\mathrm{S}(\mathrm{U}(2) \mathrm{U}(2))$ and $\mathrm{SU}(2) \not \subset \mathrm{H}_{+}$. It is clear than that $\left(\mathrm{H}_{+}\right)_{0}=\mathrm{S}^{1}$, and $\mathrm{K}_{+} / \mathrm{H}_{+}=\mathrm{SO}(4)$, implying $\mathrm{SU}(4)$ acts as $\mathrm{SO}(6)$. Switching to $\mathrm{SO}(6)$, we have $\left(\mathrm{K}_{+}\right)_{0}=\mathrm{SO}(2) \mathrm{SO}(4)$ and $\mathrm{H}_{0}=\mathrm{SO}(2) \mathrm{SO}(3)$, where $\mathrm{SO}(2)$ is the upper left and $\mathrm{SO}(3)$ and $\mathrm{SO}(4)$ are the lower right block. Since $\mathrm{SO}(2)$ must act on $\mathrm{K}_{-} / \mathrm{H}$, and the isotropy representation of $\mathrm{SO}(6) / \mathrm{H}$ decomposes in one irreducible factor of dimension 3,6 and 2 each, it's easy to deduce $\mathrm{K}_{-}=\mathrm{SO}(3) \mathrm{SO}(3)$, which gives a primitive example. Note that both $l_{ \pm}>1$, so all groups are connected.

The last case to consider is $\left(\mathrm{K}_{+}\right)_{0}=\mathrm{S}(\mathrm{U}(1) \mathrm{U}(1) \mathrm{U}(2))$, where $\mathrm{SU}(2) \not \subset \mathrm{H}_{+}$, as we have argued above. This implies $\mathrm{K}_{+} / \mathrm{H}=\mathbb{S}^{3}$, and since $\mathrm{H} / \mathrm{H}_{-}$contains $\mathrm{S}^{1}$ as a factor, we see $l_{-}>1$, so all groups are connected. We have $\mathrm{H}=\mathrm{T}^{2}$, and consider cases by the rank of $N\left(\mathrm{~K}_{-}\right)$:

- Suppose $\operatorname{rank}\left(N\left(\mathrm{~K}_{-}\right)\right)=3$. We consider the standard representation $\rho$ of $\mathrm{SU}(4)$ on $\mathbb{C}^{4}$ and its restriction to $\mathrm{K}_{ \pm}$and $\mathrm{H}$. We know $\left.\rho\right|_{\mathrm{K}_{ \pm}}$decomposes into a two-dimensional and two one-dimensional irreducible factors, while $\left.\rho\right|_{\mathrm{H}}$ decomposes into four one-dimensional irreducible factors. 
Consider for a moment the subcase that $\left.\rho\right|_{H}$ decomposes into 4 inequivalent subrepresentations $\mathbb{C} e_{1} \oplus \mathbb{C} e_{2} \oplus \mathbb{C} e_{3} \oplus \mathbb{C} e_{4}$. Then the twodimensional irreducible subspaces of $\left.\rho\right|_{\mathrm{K}_{ \pm}}$are neccessarily given by $V_{ \pm}=\mathbb{C} e_{i_{ \pm}} \oplus \mathbb{C} e_{j_{ \pm}}$for some $i_{ \pm} \neq j_{ \pm}$. If $V_{+} \cap V_{-} \neq 0$, then after permutation we can assume $V_{ \pm} \subset \mathbb{C} e_{2} \oplus \mathbb{C}_{3} \oplus \mathbb{C} e_{4}$. But that means that both $\mathrm{K}_{ \pm}$are contained in the lower $3 \times 3$-block, a contradiction. But if $V_{+} \cap V_{-}=0$, we can use a permutation again to assume $V_{+}=\mathbb{C} e_{1} \oplus \mathbb{C} e_{2}$ and $V_{-}=\mathbb{C}_{3} \oplus \mathbb{C} e_{4}$, which implies $\mathrm{K}_{ \pm} \subset \mathrm{S}(\mathrm{U}(2) \mathrm{U}(2))$, again a contradiction.

So we assume that $\left.\rho\right|_{\mathrm{H}}$ has two equivalent one-dimensional subrepresentations (note that it could not be three for any embedding $\mathrm{T}^{2} \hookrightarrow$ $\mathrm{SU}(4))$. This implies $N(\mathrm{H})=\mathrm{S}(\mathrm{U}(2) \mathrm{U}(1) \mathrm{U}(1))$, where after a permutation we may assume that $\mathbb{C} e_{1}$ and $\mathbb{C}_{2}$ are the equivalent subrepresentations of $\left.\rho\right|_{\mathrm{H}}$. Now we can argue exactly as before, using that $V_{ \pm}=A\left(\mathbb{C} e_{i_{ \pm}} \oplus \mathbb{C} e_{j_{ \pm}}\right)$for some $A \in N(\mathrm{H})_{0}$. Since we may conjugate $\mathrm{K}_{ \pm}$by any element in $N(\mathrm{H})_{0}$ without changing the manifold, we can actually assume that $V_{ \pm}=\mathbb{C} e_{i_{p} m} \oplus \mathbb{C} e_{j_{ \pm}}$and arrive at a contradiction as before. This finishes the subcase $\operatorname{rank}\left(N\left(\mathrm{~K}_{-}\right)\right)=3$ of $\left(\mathrm{K}_{+}\right)_{0}=\mathrm{S}(\mathrm{U}(1) \mathrm{U}(1) \mathrm{U}(2))$.

- The last case to consider is $\operatorname{rank}\left(N\left(\mathrm{~K}_{-}\right)\right)=2$ and $\left(\mathrm{K}_{+}\right)_{0}=\mathrm{S}(\mathrm{U}(1) \mathrm{U}(1) \mathrm{U}(2))$. This readily implies $\operatorname{rank}\left(\mathrm{K}_{-}\right)=2$ and therefore $\mathrm{K}_{-} / \mathrm{H}=\mathbb{S}^{2}$. The representation $\left.\rho\right|_{K_{-}}$(see the item before for the notation) can not be irreducible, because otherwise its restriction to the semisimple part would be irreducible as well, which would imply $\operatorname{rank}\left(\mathrm{K}_{-}\right)=1$. By virtue of $\operatorname{rank}\left(N\left(\mathrm{~K}_{-}\right)\right)=2$ it is clear that $\left.\rho\right|_{\mathrm{K}_{-}}$either decomposes into two 2dimensional irreducible subrepresentations, or into one 3-dimensional and one 1-dimensional irreducible subrepresentation. This leaves us with two cases:

a)

$$
\mathrm{K}_{-}=\left\{\left(\begin{array}{cc}
A & \\
& \bar{A}
\end{array}\right) \mid A \in \mathrm{U}(2)\right\}
$$

b) $\mathrm{K}_{-}=\mathrm{S}^{1} \mathrm{SO}(3)$ where $\mathrm{SO}(3)$ is the upper left block and

$$
\mathrm{S}^{1}=\operatorname{diag}\left(z, z, z, \bar{z}^{3}\right)
$$

In case b), we can use conjugation in $\mathrm{SO}(3)$ to achieve

$$
\mathbf{H}=\left\{\operatorname{diag}\left(z_{1}, \bar{z}_{1}, 1,1\right)\right\} \cdot\left\{\operatorname{diag}\left(z_{2}, z_{2}, z_{2}, \bar{z}_{2}^{3}\right)\right\}
$$


Thus $\left.\rho\right|_{\mathrm{H}}$ decomposes into four inequivalent one-dimensional subrepresentations $\mathbb{C}_{i}, i=1, \ldots, 4$, and the irreducible three-dimensional representation corresponding to $\left.\rho\right|_{\mathrm{K}_{-}}$is $\mathbb{C} e_{1} \oplus \mathbb{C} e_{2} \oplus \mathbb{C} e_{3}$. By primitivity, the irreducible 2-dimensional subspace of $\left.\rho\right|_{\mathrm{K}_{+}}$is given by $\mathbb{C} e_{i} \oplus \mathbb{C} e_{4}, i=$ $1, \ldots, 3$, and the whole group picture is determined by $i$. Since exchanging the first two coordinates leaves $\mathrm{K}_{-}$and $\mathrm{H}$ invariant, the cases $i=1$ and $i=2$ are equivalent, which leaves two possible examples. We claim that $\mathrm{K}_{+} / \mathrm{H}$ is not a sphere for $i=3$, but it is for $i=2$, which gives a primitive example.

$i=3$ : Since $\mathrm{SU}(2)$ acts transitively on $\mathrm{K}_{+} / \mathrm{H}$ with isotropy $\mathrm{SU}(2) \cap \mathrm{H}$, we need to show the latter is not trivial. An element of $\mathrm{H}$ being in $\mathrm{SU}(2)$ is equivalent to the three equations $z_{1} z_{2}=1, \bar{z}_{1} z_{2}=1$ and $z_{2}^{2}=1$. This is obviously true for $z_{1}=z_{2}=-1$, which constitutes a nontrivial element of $\mathrm{SU}(2) \cap \mathrm{H}$.

$i=2$ : Exchange the first an the third coordinate, moving SU(2) to the lower right block and conjugating $\mathrm{H}$ into

$$
\operatorname{diag}\left(z_{1}, 1, \bar{z}_{1}, 1\right) \operatorname{diag}\left(z_{2}, z_{2}, z_{2}, \bar{z}_{2}^{3}\right)
$$

We can read of the equations as before. This time the second coordinate show that any element of $\mathrm{H} \cap \mathrm{SU}(2)$ fulfills $z_{2}=1$, which readily implies $z_{1}=1$ from the first coordinate, so that in this case $\mathrm{H} \cap \mathrm{SU}(2)$ is trivial.

In case a), we use the fact that $-I d \in \mathrm{SU}(4)$ is in the kernel of the action, and replace $\mathrm{SU}(4)$ with $\mathrm{SO}(6)$. This gives $\mathrm{K}_{-}=\mathrm{SO}(2) \mathrm{SO}(3)$ (where $\mathrm{SO}(2)$ is in the upper left and $\mathrm{SO}(3)$ in the lower right block) and $\mathrm{H}=\mathrm{SO}(2) \mathrm{SO}(2)$, where the two trivial subrepresentation of $\rho_{\mathrm{H}}$ are spanned by $e_{3}$ and $e_{4}$. By primitivity, we have $\mathrm{K}_{+}=\mathrm{U}(2) \mathrm{SO}(2)$, giving one primitive example. 


\section{$6 \quad \mathrm{G}=\mathrm{SU}(n), n \geq 5$}

For a general overview of the classification procedure, see section 3 on page 20 . We claim that, up to equivalence, the diagrams of the simply connected primitive cohomogeneity one $\mathrm{SU}(n)$-manifolds $(n \geq 5)$ with positive euler characteristic are given by table 4 .

Table 4: SU $(n)$-cohomogeneity one manifolds for $n>4$

\begin{tabular}{c}
$\mathrm{S}^{1} \subset \sigma(\mathrm{U}(n-1)), \mathrm{U}(n-1)$ \\
where $\mathrm{S}^{1} \mathrm{SU}(n-2)=\{\operatorname{diag}(\bar{z}, z, 1, \ldots, 1)\}$ and $\sigma$ exchanges first two coordinates \\
\hline $\mathrm{S}^{1} \mathrm{SU}(n-2) \subset \mathrm{S}(\mathrm{U}(2) \mathrm{U}(n-2)), \mathrm{U}(n-1)$ \\
where $\mathrm{S}^{1}=\left\{\operatorname{diag}\left(\bar{z}^{n-2}, z^{n-2}, \bar{z}, \ldots, \bar{z}\right)\right\} \subset N(\mathrm{SU}(n-2))$ \\
\hline $\mathrm{S}^{1} \mathrm{SU}(n-2) \subset \mathrm{S}(\mathrm{U}(2) \mathrm{U}(n-2)), \mathrm{U}(n-1)$ \\
where $\mathrm{S}^{1}=\left\{\operatorname{diag}\left(\bar{z}^{n-2}, 1, z, \ldots, z\right)\right\} \subset N(\mathrm{SU}(n-2))$ \\
\hline $\mathrm{S}^{1} \mathrm{SU}(n-2) \subset \mathrm{SU}(2) \mathrm{SU}(n-2), \mathrm{U}(n-1)$ \\
where $\mathrm{S}^{1}=\{\operatorname{diag}(\bar{z}, z, 1, \ldots, 1)\} \subset N(\mathrm{SU}(n-2))$ \\
\hline $\mathrm{S}^{1} \mathrm{SU}\left(n_{1}-1\right) \mathrm{SU}\left(n_{2}\right) \subset \mathrm{S}\left(\mathrm{U}\left(n_{1}-1\right) \mathrm{U}\left(n_{2}+1\right)\right), \mathrm{S}\left(\mathrm{U}\left(n_{1}\right) \mathrm{U}\left(n_{2}\right)\right)$ \\
where $\mathrm{S}^{1}=\left\{\operatorname{diag}(\underbrace{\bar{z}^{n_{2}}, \ldots, \bar{z}^{n_{2}}}_{\times n_{1}-1}, 1, \underbrace{z^{n_{1}-1}, \ldots, z^{n_{1}-1}}_{\times n_{2}})\right.$ \\
and $\mathrm{SU}\left(n_{1}-1\right) \operatorname{SU}\left(n_{2}\right) \subset \mathrm{H}$ acts trivially on $\mathbb{C} n_{1}\left(n_{1}+n_{2}=n-1, n_{1}, n_{2}>1\right)$ \\
\hline
\end{tabular}

The possibilities for $\mathrm{K}_{+}$are summarized in table 5 (again, we refer to section 3 on page 20).

2a) In this case we assume $\mathrm{K}_{+}=\mathrm{U}(n-1)$, where we can assume $\mathrm{SU}(n-1)$ is the lower right block (this also determines the center of $\mathrm{U}(n-1)$ ). If $\mathrm{SU}(n-1) \subset \mathrm{H}_{+}$, we have $\mathrm{SU}(n) \subset \mathrm{K}_{-}$, which is treated in 1.5.2 on page 17. If $n>5$ and $\mathrm{SU}(n-1) \not \subset \mathrm{H}_{+}$, we can use table 16 on page 65 to see that $\mathrm{K}_{+} / \mathrm{H}_{+}$is $\mathrm{U}(n-1)$, so by B.2.1 on page 66 we have $\mathrm{H}=\mathrm{S}_{k}^{1} \mathrm{SU}(n-2)$ where

$$
\mathrm{S}_{k}^{1}=\left\{\operatorname{diag}\left(\bar{z}^{n-2}, z^{(k+1)(n-2)}, z^{-k}, \ldots, z^{-k}\right) \mid z \in \mathrm{S}^{1}\right\}
$$


Table 5: Possibilities for $K_{+}$

\begin{tabular}{|c|c|l|l|}
\hline Factors & Subcase & Group & Conditions \\
\hline 2 & $2 \mathrm{a}$ & $\mathrm{U}(n-1)$ & $n>2$ \\
\hline 3 & $3 \mathrm{a}$ & $\mathrm{S}\left(\mathrm{U}\left(n_{1}\right) \mathrm{U}\left(n_{2}\right)\right)$ & $n_{1}, n_{2}>1, n_{1}+n_{2}=n$ \\
\hline 3 & $3 \mathrm{~b}$ & $\mathrm{~S}^{1} \mathrm{U}(n-2)$ & $n>3$ \\
\hline 4 & $4 \mathrm{a}$ & $\mathrm{S}\left(\mathrm{U}(1) \mathrm{U}\left(n_{1}\right) \mathrm{U}\left(n_{2}\right)\right.$ & $n_{1}, n_{2}>1, n_{1}+n_{2}=n-1$ \\
\hline
\end{tabular}

For $n=5$, there is the additional possibility that $\mathrm{SU}(4)=\operatorname{Spin}(6)$ acts with isotropy $\operatorname{Spin}(5)=\mathrm{Sp}(2)$, i.e. $\mathrm{H}=\mathrm{S}^{1} \mathrm{Sp}(2)$. But $\mathrm{K}_{+} / \mathrm{H}_{+}=\mathrm{SO}(6)$ implies $\mathrm{S}^{1} \subset \mathrm{H}_{+}$, and $\mathrm{K}_{+} / \mathrm{S}^{1}=\mathrm{SO}(6) /\{ \pm I d\}$, which by table 16 on page 65 cannot act transitively on any sphere. So the above claim holds for $n=5$ as well. We differentiate between the possible values for $\mathrm{H}_{-}$(the kernel of the action of $\mathrm{K}_{-}$on $\mathrm{K}_{-} / \mathrm{H}$ ).

If $\mathrm{K}_{-}$acts almost effectively on $\mathrm{K}_{-} / \mathrm{H}$, we have $\mathrm{K}_{-} \simeq \mathrm{U}(n-1)$, and by studying the isotropy representation of $\mathrm{H}$ in $\mathrm{G}$ we see that primitivity implies $K=\sigma K_{+} \sigma^{-1}$ where

$$
\sigma=\left(\begin{array}{ccccc}
0 & 1 & & & \\
-1 & 0 & & & \\
& & 1 & & \\
& & & \ddots & \\
& & & & 1
\end{array}\right)
$$

where the empty spaces are filled up with zeroes. $\mathrm{H}$ can only occur as an isotropy group for a transitive almost effective action on a sphere of $\mathrm{K}_{-}$in the case $k=0$ or $k=-2$. In the latter case, we have $N(\mathrm{H})_{0}=\mathrm{SU}(2) \mathrm{H}$, which contains $\sigma$, so this is not a primitive example. In the former case, $\mathrm{K}_{-}$and $\mathrm{K}_{+}$are not conjugate by an element of $N(\mathrm{H})_{0}=\mathrm{T}^{2} \mathrm{SU}(n-2)$, so we get a primitive example.

The next case is $\mathrm{H}_{-}=\mathrm{S}_{k}^{1}$. Either $\mathrm{S}_{k}^{1}$ intersects $\mathrm{SU}(n-2)$ and $\mathrm{H}_{/} \mathrm{H}_{-}$ is a proper quotient of $\mathrm{SU}(n-2)$, and $\mathrm{K}_{-} / \mathrm{H}_{-}=\mathrm{SO}(7)$, which follows from studying the classification of transitive effective actions on spheres given in table 16 on page 65 . Since $N\left(\mathrm{~S}_{k}^{1}\right)$ does not contain Spin(7) or 
$\mathrm{SO}(7)$, this is impossible. The other possibility is that $\mathrm{S}_{k}^{1}$ does not intersect $\operatorname{SU}(n-2)$, which is the case exactly if $n-2$ divides $k$. Since $\mathrm{SU}(n-1) \subset N\left(\mathrm{~S}_{k}^{1}\right)$, we have $k=n-2$, which is impossible because $\mathrm{SU}(n-1) \cap \mathrm{S}_{n-2}^{1} \neq 0$.

If $\left(\mathrm{H}_{-}\right)_{0}=\mathrm{SU}(n-2)$, we have $\mathrm{H} / \mathrm{H}_{-} \simeq \mathrm{S}^{1}$ and $\mathrm{K}_{-} / \mathrm{H} \in\left\{\mathbb{S}^{2}, \mathbb{S}^{3}\right\}$. Also $\mathrm{K}_{-} \subset N(\mathrm{SU}(n-2))=\mathrm{S}^{1} \mathrm{SU}(2) \mathrm{SU}(n-2)$. If $\mathrm{K}_{-}=\mathrm{SU}(2) \mathrm{SU}(n-2)$, that implies $k=0$ and gives a primitive example. If $\mathrm{K}_{-}=N(\mathrm{SU}(n-2))$, the condition that $\mathrm{SU}(2) \cap \mathrm{H}$ is trivial is

$$
\mathrm{SU}(2) \cap \mathbf{H}=\left\{\operatorname{diag}\left(\bar{z}^{n-2}, z^{n-2}, 1, \ldots, 1\right) \mid\left(z^{k}\right)^{n-2}=1\right\} \text { is trivial }
$$

which implies $|k|=1$ and gives 2 primitive examples.

3a) For the case $\mathrm{K}_{+}=\mathrm{S}^{1} \mathrm{SU}\left(n_{1}\right) \mathrm{SU}\left(n_{2}\right)$, we will first do the most general case and after that care for the exceptional actions. Since $n_{1}, n_{2}>1$, one of $\mathrm{SU}\left(n_{1}\right), \mathrm{SU}\left(n_{2}\right)$ must act on $\mathbb{S}^{l_{+}}$, and we may assume it's $\mathrm{SU}\left(n_{1}\right)$.

Let's first show that without any further assumptions, we have $\operatorname{Sp}(2) \not \subset$ $\mathrm{H}$. Otherwise $n_{1}=4$ (and $\mathrm{S}^{1} \mathrm{SU}(4) \neq \mathrm{U}(4)$ as well, but we don't need that), and $\mathrm{H}=\mathrm{S}^{1} \mathrm{Sp}(2) \mathrm{SU}(n-4)$. This would mean $\mathrm{S}^{1} \mathrm{SU}(n-4) \subset \mathrm{H}_{-}$, but $N(\mathrm{Sp}(2))_{0}=\mathrm{H}$, so $\mathrm{K}_{-} / \mathrm{H}$ could not possibly be a sphere of positive dimension.

Now assume $n_{1}>3, n_{2}>2$. By the previous paragraph the action of $K_{+}$on $K_{+} / H$ is given as follows: The matrix $\left(\begin{array}{cc}A & 0 \\ 0 & B\end{array}\right)$ with $A \in$ $\mathrm{U}\left(n_{1}\right), B \in \mathrm{U}\left(n_{2}\right), \operatorname{det} A \operatorname{det} B=1$ acts as $(\operatorname{det} A)^{k} A$, giving

$$
H=\left\{\left(\begin{array}{ccc}
A^{\prime} & & \\
& a & \\
& & B
\end{array}\right) \mid a \operatorname{det} A^{\prime} \operatorname{det} B=1 \text { and } a^{k+1}={\overline{\operatorname{det} A^{\prime}}}^{k}\right\}
$$

for some $k \in \mathbb{Z}$. Now $\mathrm{SU}\left(n_{2}\right)$ cannot act trivially on $\mathrm{K}_{-} / \mathrm{H}$. If $n_{2}=4$, we could have $\mathrm{K}_{-} / \mathrm{H}_{-} \simeq \mathrm{SO}(7)$, but there is no $\mathrm{SO}(7)$ or $\operatorname{Spin}(7)$ in the normalizer $N(\mathrm{H})=\mathrm{S}\left(\mathrm{U}\left(n_{1}-1\right) \mathrm{U}\left(n_{2}\right)\right)$. Since $n_{2}>2$, by the classification of effective actions on spheres we have $K_{-}=\mathrm{S}^{1} \mathrm{SU}\left(n_{1}-\right.$ 1) $\mathrm{SU}\left(n_{2}+1\right)$, where $\mathbf{S U}\left(n_{1}-1\right)$ is the upper left and $\mathbf{S U}\left(n_{2}+1\right)$ the 
lower right block. The action of $K_{-}$on $K_{-} / H$ is given in a similar fashion as before, giving the isotropy group

$$
\tilde{H}=\left\{\left(\begin{array}{ccc}
A^{\prime} & & \\
& a & \\
& & B
\end{array}\right) \mid a \operatorname{det} A^{\prime} \operatorname{det} B=1 \text { and } a^{l+1}=\overline{\operatorname{det} B}^{l}\right\}
$$

for some $l \in \mathbb{Z}$. We have $\tilde{H}=H$ only in the case $k=l=0$ as follows: For any matrix in $H \cap \tilde{H}$ we have

$$
\begin{aligned}
\operatorname{det} \bar{A}^{\prime} & =a \operatorname{det} B \Rightarrow a^{k+1}=a^{k} \operatorname{det} B^{k} \Rightarrow a=\operatorname{det} B^{k} \\
\operatorname{det} \bar{B} & =a \operatorname{det} A^{\prime} \Rightarrow a^{l+1}=a^{l} \operatorname{det} A^{\prime l} \\
& \Rightarrow \operatorname{det} B^{k(l+1)}=\operatorname{det} \bar{B}^{l} \Rightarrow \operatorname{det} B^{k(l+1)+l}=1 \\
& \Rightarrow k(l+1)+l=0 \Rightarrow k=l=0 \text { because } k, l \in \mathbb{Z}
\end{aligned}
$$

giving the only primitive example in this case.

Now assume $n_{1}=2$, which by $n \geq 5$ implies $n_{2} \geq 3$. Since corank $(\mathrm{H})=$ 1 (see lemma 1.7 on page 15 ), we have $l_{+}=3$. If $\mathrm{K}_{+} / \mathrm{H}=\mathbb{S}^{3}$, we have $\mathrm{H}=\mathrm{S}^{1} \mathrm{SU}\left(n_{2}\right)$. If $n_{2}=4$, we could again have $\mathrm{K}_{-} / \mathrm{H}_{-}=\mathrm{SO}(7)$, but there's no $\mathrm{SO}(7)$ or $\mathrm{Spin}(7)$ in $\mathrm{SU}(6)$. This shows $\mathrm{K}_{-}=\mathrm{U}(n-1)$, which is a case we treated before.

The next case is $n_{1}=3$ and $n_{2} \geq 2$. But $n_{2}>2$ actually implies that the argument given in the beginning of this section applies as well, so we only need to deal with the case $n_{2}=2$. Then $\mathbf{H}=\mathbf{S}_{k}^{1} \operatorname{SU}(2) \operatorname{SU}(2)$, where

$$
\mathrm{S}_{k}^{1}=\left\{\operatorname{diag}\left(z^{2-2 k}, z^{k}, z^{k}, \bar{z}, \bar{z}\right) \mid z \in \mathrm{S}^{1}\right\}
$$

If $\mathrm{K}_{-} / \mathrm{H}=\mathbb{S}^{5}$, then again the argument above yields one primitive example (corresponding to $k=1$ here). From the classification of transitive actions on a sphere and the fact that $\mathrm{SU}(2) \subset \mathrm{H} / \mathrm{H}_{-}$, we are left with 2 further possibilities: $\mathrm{K}_{-} / \mathrm{H}_{-}=\mathrm{SO}(5)$ and $\mathrm{K}_{-} / \mathrm{H}_{-}=$ $\mathrm{SO}(4)$.In the first case we can deduce $\mathrm{K}_{-}=\mathrm{S}^{1} \mathrm{Sp}(2)$, which implies $k=-1$, but then $\mathrm{K}_{+} / \mathrm{H}=\mathbb{R} \mathbb{P}^{5}$. In the latter case, we would have $\mathrm{SU}(2) \subset \mathrm{H}_{-}$, but there is no $\mathrm{SO}(4)$ or $\operatorname{Spin}(4)=\mathrm{SU}(2) \times \mathrm{SU}(2)$ in the normalizer $N(\mathrm{SU}(2))$ in $\mathrm{SU}(5)$. 
The last case to consider is $n_{1}>3, n_{2}=2$. We will argue that there is no additional possibility to the one given at the beginning of this section. We have $\mathrm{H}=\mathrm{S}^{1} \mathrm{SU}(n-3) \mathrm{SU}(2)$, and since $n-3 \geq 3$ we have $\mathrm{SU}(n-3) \subset \mathrm{H}_{-}$. But $N(\mathrm{SU}(n-3))=\mathrm{S}^{1} \mathrm{SU}(3)$, so the only possibility is $\mathrm{SU}(3) \subset \mathrm{K}_{-} / \mathrm{H}_{-}$, which is the aforementioned argument.

4a) We assume $\mathrm{SU}\left(n_{1}\right)$ is the upper left, $\mathrm{SU}\left(n_{2}\right)$ the lower right block. Note that this determins the center $\mathrm{T}^{2}$ of $\mathrm{K}_{+}$. By remark 1.9 on page 16 and the fact that in this case $\mathrm{K}_{+}$has 4 factors, we can assume $\left(\mathrm{H}_{-}\right)_{0}=$ $\mathrm{S}^{1} \mathrm{SU}\left(n_{2}\right)$ and therefore $\mathrm{H}=\mathrm{T}^{2} \mathrm{SU}\left(n_{1}-1\right) \operatorname{SU}\left(n_{2}\right)$, where $\mathrm{SU}\left(n_{1}-1\right)$ is the upper left and $\mathrm{SU}\left(n_{2}\right)$ is the lower right block. If we assume $\mathrm{K}_{-} \simeq \mathrm{U}\left(n_{1}-1\right) \mathrm{U}\left(n_{2}+1\right)$ and $n_{1}>2$ then we know the resulting manifold will not be primitive for the following reason: $\mathrm{K}_{-} \hookrightarrow \mathrm{SU}(n)$ induces a 1-dimensional subrepresentation, which by the choice of $\mathrm{K}_{+}$ and $\mathrm{H}$ is necessarily given by $e_{n_{1}}$ or $e_{n_{1}+1}$. The first case implies $\mathrm{K}_{ \pm} \subset$ $\mathrm{S}^{1} \mathrm{SU}\left(n_{1}\right) \mathrm{SU}\left(n_{2}+1\right)$, and the second $\mathrm{K}_{ \pm} \subset \mathrm{U}(n-1)$.

We divide the remainig cases:

- If $n_{1}=2$, then $\mathrm{H}=\mathrm{T}^{2} \mathrm{SU}\left(n_{2}\right)$, and $\mathrm{H} \hookrightarrow \mathrm{SU}(n)$ induces 3 onedimensional subrepresentations via the standard representation. We know $\mathrm{K}_{-} \simeq \mathrm{T}^{2} \mathrm{SU}\left(n_{2}+1\right)$, for which we only need to note that $\mathrm{T}^{2} \mathrm{Sp}(2)$ is not a subgroup of $\mathrm{SU}(4)$ by table 15 on page 65 . Now $\mathrm{K}_{-} \hookrightarrow \mathrm{SU}(n)$ induces 2 one-dimensional subrepresentations, and if one of those is given by $e_{3}$, we have $\mathrm{K}_{ \pm} \subset \mathrm{U}(n-1)$. But in the other case $\mathrm{K}_{ \pm} \subset \mathrm{S}^{1} \mathrm{SU}(2) \mathrm{SU}(n-2)$, so there is no primitive example in this case.

- We will now argue that indeed $\mathrm{K}_{-} \simeq \mathrm{U}\left(n_{1}-1\right) \mathrm{U}\left(n_{2}+1\right)$ in all other cases. By primitivity we know $\left(\mathrm{H} / \mathrm{H}_{-}\right)_{0}=\mathrm{S}^{1} \mathrm{SU}\left(n_{2}\right)$, so by the classification given in table 16 on page 65 this is true for $n_{1} \geq 3, n_{2} \geq 3$. Having already dealt with the case $n_{1}=2$, we only need to consider $n_{1} \geq 3, n_{2}=2$, but again we only need to note that $\mathrm{T}^{2} \mathrm{Sp}(2) \mathrm{SU}\left(n_{1}-4\right)$ is not a subgroup of $\mathrm{SU}(n)$ by table 15 on page 65 . This shows $\mathrm{K}_{-} \simeq \mathrm{U}\left(n_{1}-1\right) \mathrm{U}\left(n_{2}+1\right)$ as desired, and we know that the manifold is not primitive by what was said above.

3b) If $\mathrm{SU}(n-2) \subset \mathrm{H}_{+}$, we have $\mathrm{SU}(n-1) \subset \mathrm{K}_{-}$(remember that $n \geq 5$ ), so that $\mathrm{K}_{-} \simeq \mathrm{U}(n-1)$, which was treated before. So now assume $\mathrm{SU}(n-2) \not \subset \mathrm{H}_{+}$, which implies $\mathrm{H}=\mathrm{T}^{2} \mathrm{SU}(n-3)$. We distinguish the cases $\mathrm{SU}(n-3) \not \subset \mathrm{H}_{-}$and its opposite.

- If $\mathrm{SU}(n-3) \not \subset \mathrm{H}_{-}$, we have $\mathrm{K}_{-} \simeq \mathrm{K}_{+}$. The factor $\mathrm{SU}(n-2)$ of $\mathrm{K}_{-}$then has a 2-dimensional trivial subrepresentation when 
consideres as a subgroup of $\mathrm{SU}(n)$ which is necessarily given by $\mathbb{C} e_{i} \oplus \mathbb{C} e_{j}$ for $i, j \in\{1,2,3\}$ :

* If $i=1, j=2$, we have $\mathrm{K}_{-}=\mathrm{K}_{+}$, so the example is not primitive.

* If $i=1, j=3$, we have $\mathrm{K}_{ \pm} \subset \mathrm{S}(\mathrm{U}(1) \mathrm{U}(n-1))$, so again this is not primitive

$*$ If $i=2, j=3$, exchange the coordinates $e_{1}$ and $e_{2}$, after which we are in the previous case again. Note that this exchange might change every group of the diagram, yet still shows that it is not primitive.

- If $\mathrm{SU}(n-3) \subset \mathrm{H}_{-}$, we have $\mathrm{H} / \mathrm{H}_{-} \simeq \mathrm{S}^{1}$, which implies $\mathrm{K}_{-} / \mathrm{H}_{-}$ is one of $\mathrm{U}(2), \mathrm{SO}(3)$. In the first case $\mathrm{K}_{-} \simeq \mathrm{S}(\mathrm{U}(2) \mathrm{U}(n-3))$, which was previously treated in case $4 \mathrm{a}$. In the latter case, we have $\mathrm{K}_{-} \simeq \mathrm{SO}(3) \mathrm{U}(n-3)$, and we conjugate the whole diagram to make $\mathrm{K}_{-}$standard: $\mathrm{SO}(3)$ is the upper left block, $\mathrm{SU}(n-3)$ the lower right and $\mathrm{S}^{1}$ is diagonally embedded, so it commutes with both $\mathrm{SO}(3)$ and $\mathrm{SU}(n-3)$ but does not act trivially on any of the $\mathbb{C} e_{i}$ for $i \in\{1 \ldots, n\}$. We can assume $\mathrm{H}$ is then given by $\mathrm{S}_{1}^{1} \mathrm{~S}_{2}^{1} \mathrm{SU}(n-3)$ where

$$
\mathrm{S}_{1}^{1}=\left\{\operatorname{diag}\left(z_{1}, 1, \bar{z}_{1}, 1, \ldots, 1\right) \mid z_{1} \in \mathrm{S}^{1}\right\}
$$

and

$$
\mathrm{S}_{2}^{1}=\left\{\operatorname{diag}\left(\bar{z}_{2}^{n-3}, \bar{z}_{2}^{n-3}, \bar{z}_{2}^{n-3}, z_{2}^{3}, \ldots, z_{2}^{3}\right) \mid z_{2} \in \mathrm{S}^{1}\right\}
$$

Now $\mathrm{K}_{+}=\mathrm{S}(\mathrm{U}(1) \mathrm{U}(1) \mathrm{U}(n-2))$ is determined by $\mathrm{SU}(n-2)$, which again has a 2-dimensional trivial subrepresentation when restricting the standard representation of $\mathrm{SU}(n)$, which is necessarily given by $\mathbb{C} e_{i} \oplus \mathbb{C} e_{j}$ for $i, j \in\{1,2,3\}$ and $i \neq j$. Note that the cases $i=1, j=2$ and $i=2, j=3$ are equivalent, and we claim this gives a (primitive) example. The last case $i=1, j=3$ does not give an example for $\mathrm{K}_{+} / \mathrm{H}$ is not a sphere:

* If $i=1, j=2$, an element of $\mathrm{H}$ that is also in $\mathrm{SU}(n-2)$ satisfies $z_{1} \bar{z}_{2}^{n-3}=1$ and $\bar{z}_{2}^{n-3}$ from the first two coordinates, which implies $z_{1}=1$ and so $\operatorname{diag}\left(1,1,1, z_{2}^{3}, \ldots, z_{2}^{3}\right) \in \operatorname{SU}(n-3)$, which shows $\mathrm{SU}(n-2) \cap \mathrm{H}=\mathrm{SU}(n-3)$, so $\mathrm{K}_{+} / \mathrm{H}=\mathrm{SU}(n-$ $2) /(\mathrm{H} \cap \mathrm{SU}(n-2))$ is a sphere.

* If $i=1, j=3$, an element of $\mathrm{H}$ is in $\mathrm{SU}(n-2)$ if and only if $z_{1} \bar{z}_{2}^{n-3}=1$ and $\bar{z}_{1} \bar{z}_{2}^{n-3}$ from the first and the third coordinate. 
Choose any $z_{2}$ such that $z_{2}^{n-3}=-1$ and $z_{1}=-1$, so that these equations are fulfilled. But then note that the second coordinate of this element is -1 , which shows that it is in $\mathrm{H} \cap \mathrm{SU}(n-2)$, but not in $\mathrm{SU}(n-3)$. Therefore $\mathrm{K}_{+} / \mathrm{H}=$ $\mathrm{SU}(n-2) /(\mathrm{H} \cap \mathrm{SU}(n-2))$ is not a sphere. 


\section{$7 \quad \mathrm{G}=\mathrm{SO}(2 n+1), n \geq 3$}

For the general procedure of the classification see section 3 on page 20 . Up to equivalence, the simply connected primitive cohomogeneity one $\mathrm{SO}(2 n+1)$ manifolds $(n \geq 3)$ are given by table 6 .

Table 6: $\mathbf{S O}(2 n+1)$-cohomogeneity one manifolds for $n>2$

\begin{tabular}{|c|}
\hline $\mathrm{SO}(2 n-1) \subset \mathrm{SO}(2) \mathrm{SO}(2 n-1), \mathrm{SO}(2 n)$ \\
\hline $\mathrm{O}(2 n-1) \subset \mathrm{SO}(2) \mathrm{SO}(2 n-1), \mathrm{O}(2 n)$ \\
\hline $\begin{array}{r}\mathrm{SO}\left(2 n_{1}+1\right) \mathrm{SO}\left(2 n_{2}-1\right) \subset \mathrm{SO}\left(2 n_{1}+2\right) \mathrm{SO}\left(2 n_{2}-1\right), \mathrm{SO}\left(2 n_{1}+1\right) \mathrm{SO}\left(2 n_{2}\right) \\
\text { where } n_{1}+n_{2}=n\end{array}$ \\
\hline $\mathrm{SO}(2) \mathrm{SO}(2 n-3) \subset \mathrm{U}(2) \mathrm{SO}(2 n-3), \mathrm{SO}(2) \mathrm{SO}(2 n-2)$ \\
\hline $\mathrm{SO}\left(2 n_{1}-1\right) \mathrm{U}\left(n_{2}\right) \subset \mathrm{SO}\left(2 n_{1}-1\right) \mathrm{U}\left(n_{2}+1\right), \mathrm{SO}\left(2 n_{1}\right) \mathrm{U}\left(n_{2}\right)$ \\
where $n_{1}+n_{2}=n$
\end{tabular}

The possibilities for $K_{+}$are given by table 7 (again we refer to section 3 on page 20 .

If a group of complex matrices is involved (e.g. $\mathrm{U}(n)$ ), we will deliberatly use complex notation for the corresponding real matrices. In particular, for $e^{i \varphi}=z \in S^{1}$, we will use $\operatorname{diag}(z, \ldots, z)$ for the matrix containing $\left(\begin{array}{cc}\cos \varphi & \sin \varphi \\ -\sin \varphi & \cos \varphi\end{array}\right)$ on the diagonal $2 \times 2$-blocks and 0 everywhere else.

1a) We have $\mathbf{H}=\mathrm{SO}(2 n-1)$. There are two possibilities for $\mathrm{K}_{-}$, namely $\mathrm{K}_{-} \simeq \mathrm{SO}(2 n)$ and $\mathrm{K}_{-} \simeq \mathrm{SO}(2) \mathrm{SO}(2 n-1)$. The first choice does not lead to a primitive manifold, since either $\mathrm{K}_{-}=\mathrm{K}_{+}$or $\mathrm{K}_{-}$is conjugate to $\mathrm{K}_{+}$via the matrix 
Table 7: Possibilities for $K_{+}$

\begin{tabular}{|c|c|l|l|}
\hline Factors & Subcase & Group & Conditions \\
\hline 1 & $1 \mathrm{a}$ & $\mathrm{SO}(2 n)$ & - \\
\hline 2 & $2 \mathrm{a}$ & $\mathrm{SO}\left(2 n_{1}\right) \mathrm{SO}\left(2 n_{2}\right)$ & $n_{1}, n_{2} \geq 3, n_{1}+n_{2}=n$ \\
\hline 2 & $2 \mathrm{~b}$ & $\mathrm{SO}\left(2 n_{1}+1\right) \mathrm{SO}\left(2 n_{2}\right)$ & $n_{2} \geq 2, n_{2}+n_{2}=n$ \\
\hline 2 & $2 \mathrm{c}$ & $\mathrm{SO}(2) \mathrm{SO}(2 n-2)$ & $n \geq 4$ \\
\hline 2 & $2 \mathrm{~d}$ & $\mathrm{SO}(2) \mathrm{SO}(2 n-1)$ & - \\
\hline 2 & $2 \mathrm{e}$ & $\mathrm{U}(n)$ & - \\
\hline 3 & $3 \mathrm{a}$ & $\mathrm{SO}\left(2 n_{1}\right) \mathrm{U}\left(n_{2}\right)$ & $n_{1}, n_{2} \geq 2, n_{1}+n_{2}=n$ \\
\hline 3 & $3 \mathrm{~b}$ & $\mathrm{SO}\left(2 n_{1}+1\right) \mathrm{U}\left(n_{2}\right)$ & $n_{2} \geq 2, n_{1}+n_{2}=n$ \\
\hline 3 & $3 \mathrm{c}$ & $\mathrm{SO}(2) \mathrm{U}(n-1)$ & - \\
\hline 3 & $3 \mathrm{~d}$ & $\mathrm{SO}(2 n-4) \mathrm{SO}(4)$ & $n \geq 5$ \\
\hline 3 & $3 \mathrm{e}$ & $\mathrm{SO}(2) \mathrm{SO}(4)$ & $n=3$ \\
\hline 3 & $3 \mathrm{f}$ & $\mathrm{SO}(2 n-3) \mathrm{SO}(4)$ & - \\
\hline 4 & $4 \mathrm{a}$ & $\mathrm{U}\left(n_{1}\right) \mathrm{U}\left(n_{2}\right)$ & $n_{1}, n_{2} \geq 2, n_{1}+n_{2}=n$ \\
\hline 4 & $4 \mathrm{~b}$ & $\mathrm{U}(n-2) \mathrm{SO}(4)$ & $n \geq 4$ \\
\hline 4 & $4 \mathrm{c}$ & $\mathrm{SO}(4) \mathrm{SO}(4)$ & $n=4$ \\
\hline & & & \\
\hline
\end{tabular}

$$
\left(\begin{array}{cccc}
0 & 1 & 0 & \\
-1 & 0 & 0 & \\
& & & \\
& & & E_{2 n-1}
\end{array}\right)
$$

which exchanges the first two coordinates and is in $N(\mathrm{H})_{0}$. There is only one $\mathrm{SO}(2)$ in the normalizer of $\mathrm{SO}(2 n-1)$, so the second choice leads to exactly one primitive manifold.

We do have $l_{-}=0$ here, so $\mathrm{K}_{+}$might be non-connected. It is clear then that $\mathrm{H}=\mathbb{Z}_{2} \mathrm{SO}(2 n-1) \simeq \mathrm{O}(2 n-1)$ and $\mathrm{K}_{+}=\mathbb{Z}_{2} \mathrm{SO}(2 n) \simeq \mathrm{O}(2 n)$. 
2a) We can assume $\mathrm{H}=\mathrm{SO}\left(2 n_{1}-1\right) \mathrm{SO}\left(2 n_{2}\right)$, and it is clear that $\mathrm{K}_{-} \simeq$ $\mathrm{SO}\left(2 n_{1}-1\right) \mathrm{SO}\left(2 n_{2}+1\right)$. There are two possibilities for $\mathrm{SO}\left(2 n_{2}+1\right)$ in the normalizer of $\mathrm{SO}\left(2 n_{1}-1\right)$, both of which are conjugate by a change of coordinate, which can be achieved by conjugation with a matrix similar to the one given in $1 \mathrm{a}$, which is in $N(\mathrm{H})_{0}=\mathrm{SO}(2) \mathrm{SO}\left(2 n_{1}-\right.$ 1) $\mathrm{SO}\left(2 n_{2}\right)$. But it is obvious for at least one of the two possibilities that $\mathrm{K}_{ \pm} \subset \mathrm{SO}(2 n)$.

2b) Since corank $(\mathrm{H})=1$ by corollary 1.7 on page 15 , we have $\mathrm{H}=\mathrm{SO}\left(2 n_{1}-\right.$ 1) $\mathrm{SO}\left(2 n_{2}+1\right)$, implying $K_{-}=\mathrm{SO}\left(2 n_{1}-1\right) \mathrm{SO}\left(2 n_{2}+2\right)$. This gives one primitive example.

2c) First assume $\mathrm{H}=\mathrm{SO}(2) \mathrm{SO}(2 n-3)$. Further assuming $\mathrm{K}_{-} / \mathrm{H}_{-}=\mathrm{U}(2)$, we see $\mathrm{K}_{-} \simeq \mathrm{U}(2) \mathrm{SO}(2 n-3)$. The choices for $\mathrm{U}(2)$ in the normalizer of $\mathrm{SO}(2 n-3)$ are given by the center, which is given by either $\left\{\operatorname{diag}(z, z, 1, \ldots, 1) \mid z \in \mathrm{S}^{1}\right\}$ or $\left\{\operatorname{diag}(\bar{z}, z, 1, \ldots, 1) \mid z \in \mathrm{S}^{1}\right\}$. But complex conjugation of the first component in $\mathrm{T}^{2} \subset \mathrm{SO}(4)$ is given by conjugation with $\operatorname{diag}(-1,1, \ldots, 1)$, which leaves $\mathrm{H}$ invariant as well as $\mathrm{K}_{+}$, so we only get one new example.

The next possibility is $\mathrm{K}_{-} \simeq \mathrm{SO}(3) \mathrm{SO}(2 n-3)$, and again from checking the isotropy representation there are 2 choices for $\mathrm{SO}(3)$, but both are conjugate by a change of two coordinates, which is in $N(\mathrm{H})_{0}$ as in 1 a. The result is not primitive.

If $\mathrm{H}=\mathbb{Z}_{k} \mathrm{SO}(2 n-2)$, we have $\mathrm{K}_{-}=\mathbb{Z}_{k} \mathrm{SO}(2 n-1)$. In the case that $\mathrm{H}$ is connected, there are several choices for $\mathrm{K}_{-}=\mathrm{SO}(2 n-1)$, but all of them are conjugate in $N(\mathrm{H})_{0}=\mathrm{SO}(3) \mathrm{SO}(2 n-2)$, and obviously not primitive. If $\mathrm{H}$ is not connected, there's only one choice for $\mathrm{SO}(2 n-1)$, but again $\mathrm{K}_{ \pm} \subset \mathrm{SO}(2) \mathrm{SO}(2 n-1)$.

2d) We only need to note $\mathrm{SO}(2 n-1) \subset \mathrm{H}_{+}$, since $\operatorname{corank}(\mathrm{H})=1$ by corollary 1.7 on page 15 , which leads to $\left(\mathrm{K}_{-}\right)_{0}=\mathrm{SO}(2 n)$ which was treated in 1a.

2e) We cannot have $\mathrm{SU}(n) \subset \mathrm{H}_{+}$, for that would imply $\mathrm{SU}(n+1) \subset \mathrm{K}_{-}$, but there is no embedding of $\mathrm{SU}(n+1) \hookrightarrow \mathrm{SO}(2 n+1)$ (as seen easily from checking representations). So we know $\mathrm{H}=\mathrm{S}_{k}^{1} \mathrm{SU}(n-1)$, where

$$
\mathrm{S}_{k}^{1}=\left\{\operatorname{diag}\left(1, z^{(k+1)(n-1)}, z^{-k}, \ldots, z^{-k}\right) \mid z \in \mathrm{S}^{1}\right\}
$$

and $\mathrm{SU}(n-1)$ is in the lower right block. 
First assume $k=-1$. We have $N(\mathrm{H})_{0}=\mathrm{SO}(3) \mathrm{SU}(n-1)$. If $\mathrm{K}_{-} \simeq \mathrm{U}(n)$, there are several choices, but all of them can be conjugated into $\mathrm{SO}(2 n)$ by a simple change of coordinates, which is in $N(\mathrm{H})_{0}$, so there's no primitive example. We cannot have $\mathrm{SU}(n-1) \subset \mathrm{H}_{-}$, but $\mathrm{S}^{1} \not \subset \mathrm{H}_{-}$, since there are no subgroups in $N(\mathrm{SU}(n-1))$ of type $\mathrm{U}(2), \mathrm{SO}(3), \mathrm{SU}(2)$ containing $\mathrm{S}_{k}^{1}$. Lastly $\mathrm{U}(n-1) \subset \mathrm{H}_{-}$implies $\mathrm{K}_{-} \simeq \mathrm{SO}(2) \mathrm{U}(n-1)$, and again conjugation in $N(\mathrm{H})_{0}$ leads to $\mathrm{K}_{-} \subset \mathrm{K}_{+}$.

Now for $k \neq-1$ we have $N(\mathrm{H})_{0}=\mathrm{SO}(2) \mathrm{U}(n-1)$. If $\mathrm{K}_{-} \simeq \mathrm{U}(n)$ there are two choices for embedding $\mathrm{U}(n)$, but both are in $\mathrm{SO}(2 n)$ as well as $\mathrm{K}_{+}$. We cannot have $\mathrm{H}=\mathrm{H}_{-}$, because $N(\mathrm{H})_{0} \subset \mathrm{U}(n)$, so we would not get a primitive manifold. For the same reasons as before, $\mathrm{SU}(n-1) \subset \mathrm{H}_{-}$, but $\mathrm{S}_{k}^{1} \not \subset \mathrm{H}_{-}$is not possible

3a) If $\mathrm{H}=\mathrm{SO}\left(2 n_{1}-1\right) \mathrm{U}\left(n_{2}\right)$, it is clear that $\mathrm{K}_{-} \simeq \mathrm{SO}\left(2 n_{1}-1\right) \mathrm{U}\left(n_{2}+1\right)$, and there are 2 choices for embedding $\mathrm{K}_{-}$into $\mathrm{SO}(2 n+1)$ while containing $\mathrm{H}$, corresponding to the embedding of the additional coordinate of its center over the one of $\mathrm{H}$. Both are conjugate by

$$
\operatorname{diag}(\underbrace{1, \ldots, 1}_{2 n_{1}-1},-1, \underbrace{1, \ldots, 1}_{2 n_{2}+2})
$$

where we assume that $\mathrm{U}\left(n_{2}\right)$ is embedded as the lower right block, and $\mathrm{SO}\left(2 n_{1}-1\right)$ in the upper left. This conjugation is not in $N(\mathrm{H})_{0}=$ $\mathrm{SO}(2) \mathrm{H}$, but restricted to $\mathrm{K}_{-}$it is the same as conjugation with

$$
\operatorname{diag}(\underbrace{-1, \ldots,-1}_{2 n_{1}-1},-1, \underbrace{1, \ldots, 1}_{2 n_{2}+2})
$$

which is in $\mathrm{SO}\left(2 n_{1}\right)$ and leaves both $\mathrm{K}_{+}$and $\mathrm{H}$ invariant. So we only obtain one example, which is primitive, because $\mathrm{K}_{-}$is a maximal subgroup of maximal rank not containing and not contained in $\mathrm{K}_{+}$(and not isomorphic to it).

The second possibility is $\mathrm{H}=\mathrm{SO}\left(2 n_{1}\right) \mathrm{U}\left(n_{2}-1\right)_{k}$ (where $\mathrm{U}\left(n_{2}-1\right)_{k}=$ $\mathrm{S}_{k}^{1} \mathrm{SU}\left(n_{2}-1\right)$ similar to $\left.2 \mathrm{a}\right)$. It is clear that $\mathrm{K}_{-} \simeq \mathrm{SO}\left(2 n_{1}+1\right) \mathrm{U}\left(n_{2}-1\right)_{k}$, and for $k \neq-1$ there is just one possibility for that, which is not primitive, for $\mathrm{K}_{ \pm} \subset \mathrm{SO}\left(2 n_{1}+1\right) \mathrm{SO}\left(2 n_{2}\right)$. If $k=-1$, there are actually 3 possibilities, but all again differ only by a change of coordinates, which can be done in $N(\mathrm{H})_{0}=\mathrm{SO}(3) \mathrm{H}$, so again no primitive example arises. 
3b) We cannot have $\mathrm{H}_{+}=\mathrm{U}\left(n_{2}\right)$, for that would imply $\mathrm{SO}\left(2 n_{1}\right) \mathrm{U}\left(n_{2}+1\right) \subset$ $\mathrm{K}_{-}$, but this can't be embedded into $\mathrm{SO}(2 n+1)$. Also, $\mathrm{H}_{+} \neq \mathbb{Z}_{k} \mathrm{SU}\left(n_{2}\right)$ for any $k$, because $\mathrm{SO}\left(2 n_{1}+1\right) \mathrm{SU}\left(n_{2}\right)$ is not isotropy group of any almost effective transitive action on a sphere.

So we have $\mathrm{H}=\mathrm{SO}\left(2 n_{1}+1\right) \mathrm{U}\left(n_{2}-1\right)_{k}$ (see $3 \mathrm{~b}$ for notation), and therefore $\mathrm{SO}\left(2 n_{1}+2\right) \subset \mathrm{K}_{-}$is in the normalizer of $\mathrm{U}\left(n_{2}-1\right)_{k}$, which is only possible for $k=-1$. If $k=-1$, we have 2 possibilities for $\mathrm{K}_{-}$, both conjugate by a change of coordinates, which is in $\mathbb{N}(H)_{0}=\mathrm{SO}(2) \mathrm{H}$. We are left with $\mathrm{K}_{-}=\mathrm{SO}\left(2 n_{1}+2\right) \mathrm{U}\left(n_{2}-1\right)$, which is primitive as shown in $3 \mathrm{a}$.

3c) First suppose $\mathrm{SU}(n-1) \subset \mathrm{H}_{+}$. This implies $\mathrm{SU}(n) \subset \mathrm{K}_{-}$by primitivity and the classification of transitive actions on spheres. Therefore $\left(\mathrm{K}_{-}\right)_{0} \simeq \mathrm{U}(n)$, which is contained in case 2e. From now on we assume $\mathrm{SU}(n-1) \not \subset \mathrm{H}_{+}$.

By remark 1.9 on page 16 we can deduce $\mathrm{H}_{0}=\mathrm{T}^{2} \mathrm{SU}(n-2)$. Suppose $\mathrm{SU}(n-2) \not \subset \mathrm{H}_{-}$(note that this implies $n>3$ ). Then $\mathrm{K}_{-} / \mathrm{H}_{-} \simeq$ $\mathrm{S}^{1} \mathrm{SU}(n-1)$. Now we can deduce $\mathrm{K}_{ \pm} \subset \mathrm{SO}(2 n)$ as follows: All irreducible real representations of $\mathrm{K}_{ \pm}$and $\mathrm{H}$ are even-dimensional, so there is at least one 1-dimensional trivial representation given by the embedding $\mathrm{K}_{-} \hookrightarrow \mathrm{SO}(2 n+1)$, which of course stays trivial when restricted to $\mathrm{H}$. If the embedding $\mathrm{H} \hookrightarrow \mathrm{SO}(2 n+1)$ induces only one such representation, then this is neccessarily the same as the one for $\mathrm{K}_{+}$, which shows the claim. If $\mathrm{H} \hookrightarrow \mathrm{SO}(2 n+1)$ induces three trivial 1-dimensional representations, then $N(\mathrm{H})_{0} \supset \mathrm{SO}(3)$, and we may again assume $\mathrm{K}_{-} \subset \mathrm{SO}(2 n)$ by section 1.2 on page 13 .

Now we are left with the case $\mathbf{H}=\mathrm{T}^{2} \mathrm{SU}(n-2)$ and $\mathrm{SU}(n-2) \subset \mathrm{H}_{-}$, which implies $l_{-}=2$ or $l_{-}=3$. We divide cases by the possibilities for $\mathrm{K}_{-}$:

- Suppose $l_{-}=2, \mathrm{~K}_{-} \simeq \mathrm{SO}(3) \mathrm{S}^{1} \mathrm{SU}(n-2)$ and $\mathrm{SO}(3) \hookrightarrow \mathrm{SO}(2 n+1)$ is irreducible in $\mathrm{SO}(5) \subset N\left(\mathrm{~S}^{1} \mathrm{SU}(n-2)\right)$. We note that $\mathrm{SO}(2) \subset$ $\mathrm{SO}(3)$ has weights 1 and 2 in $\mathrm{SO}(5)$ in this case, and therefore we have $\mathrm{H}=\mathrm{S}_{1}^{1} \mathrm{~S}_{2}^{1} \mathrm{SU}(n-2)$, where $\mathrm{SU}(n-2)$ is the lower right block and

$$
\begin{aligned}
& \mathrm{S}_{1}^{1}=\left\{\operatorname{diag}\left(1, z, z^{2}, 1, \ldots, 1\right) \mid z \in \mathrm{S}^{1}\right\} \\
& \mathrm{S}_{2}^{1}=\left\{\operatorname{diag}(\underbrace{1, \ldots, 1}_{5}, \underbrace{z, \ldots, z}_{n-2} \mid z \in \mathrm{S}^{1}\}\right.
\end{aligned}
$$


This yields one primitive example.

- If $l_{-}=2, \mathrm{~K}_{-} \simeq \mathrm{SO}(3) \mathrm{S}^{1} \mathrm{SU}(n-2)$ and $\mathrm{SO}(3) \hookrightarrow \mathrm{SO}(5) \subset$ $N(\mathrm{SU}(n-2))$ has a trivial 2-dimensional subrepresentation, then we can reconjugate the diagram to achieve $\mathrm{H}=\mathrm{S}_{1}^{1} \mathrm{~S}_{2}^{1} \mathrm{SU}(n-2)$ where $\mathrm{SU}(n-2)$ is the lower right block and

$$
\begin{gathered}
\mathrm{S}_{1}^{1} \quad=\left\{\operatorname{diag}(1, z, 1, \ldots, 1) \mid z \in \mathrm{S}^{1}\right\} \\
\mathrm{S}_{2}^{1}=\left\{\operatorname{diag}\left(1,1,1, z^{l_{2}}, z^{k_{2}}, \ldots, z^{k_{2}}\right) \mid z \in \mathrm{S}^{1}\right\}
\end{gathered}
$$

where $l_{2}, k_{2} \in \mathbb{Z}$. The trivial 1-dimensional subrepresentation of $\mathrm{K}_{+} \hookrightarrow \mathrm{SO}(2 n+1)$ is then neccessarily given by $\mathbb{R} e_{1}$, which shows $\mathrm{K}_{ \pm} \subset \mathrm{SO}(3) \mathrm{U}(n-2)$, and there is no primitive example in this case.

- If $l_{-}=2$ and $\mathrm{K}_{-} \simeq \mathrm{SU}(2) \mathrm{S}^{1} \mathrm{SU}(n-2)$, we have $\mathrm{K}_{ \pm} \subset \mathrm{SO}(2 n)$ by the same reasoning that we used in the first paragraph of this case.

- If $l_{-}=3$, then $\mathrm{K}_{-} \simeq \mathrm{U}(2) \mathrm{U}(n-2)$ and again $\mathrm{K}_{ \pm} \subset \mathrm{SO}(2 n)$ by the same reasoning that we used in the first paragraph of this case.

3d-f) All these are subject to the considerations in $2 \mathrm{a}$ and $2 \mathrm{~b}$, since the corank of $\mathrm{H}$ in $\mathrm{G}$ is 1 by corollary 1.7 on page 15 , so that $\mathrm{K}_{+} / \mathrm{H}$ is not $\mathrm{S}^{2}$.

4a) We can assume $\mathrm{H}=\mathrm{T}^{2} \mathrm{SU}\left(n_{1}-1\right) \mathrm{SU}\left(n_{2}\right)$ and therefore $\mathrm{K}_{-}=\mathrm{T}^{2} \mathrm{SU}\left(n_{1}-\right.$ $1) \mathrm{SU}\left(n_{2}+1\right)$. We will identify $\mathrm{K}_{-}$via its center. It has to commute with $\mathrm{SU}\left(n_{1}-1\right) \mathrm{SU}\left(n_{2}\right)$, so we need to look at embeddings $\mathrm{S}^{1} \hookrightarrow \mathrm{SO}(3)$, all of which are conjugate in $\mathrm{SO}(3)$ up to complex conjugation. But this implies that $\mathrm{K}_{ \pm} \subset \mathrm{SO}(2 n)$ after conjugating $\mathrm{K}_{-}$with an element of $N(\mathrm{H})_{0}$, which contains the $\mathrm{SO}(3)$ in question.

4a-b) Again, we have corank $(\mathrm{H})=1$ by corollary 1.7 on page 15 , so in particular $\mathrm{K}_{+} / \mathrm{H} \neq \mathrm{S}^{2}$. All the other cases have been treated before.

\section{1 $\mathrm{G}=\operatorname{Spin}(2 n+1), n \geq 3$}

For the general procedure of the classification see section 3 on page 20. For comments on the procedure for the spin groups, see 3.1 on page 20 . Up to equivalence, the simply connected primitive cohomogeneity one $\operatorname{Spin}(2 n+1)$ manifolds $(n \geq 3)$ are given by table 8 .

The possibilities for $\mathrm{K}_{+}$are given by table 9 . 
Table 8: $\operatorname{Spin}(2 n+1)$-cohomogeneity one manifolds for $n>2$

\begin{tabular}{c}
\hline $\mathrm{S}_{k}^{1} \mathrm{SU}(2) \subset \mathrm{U}(3), \mathrm{SO}(2) \mathrm{SO}(5), n=3$ \\
where $\mathrm{S}_{k}^{1}=\left\{\operatorname{diag}\left(z^{2}, z^{k}, z^{k}, 1\right)\right\}, k=1,-3$ and \\
$\mathrm{SU}(2)$ acts trivially on $\mathbb{R} e_{1}, \mathbb{R} e_{2}, \mathbb{R} e_{7}$ \\
\hline $\mathrm{SU}(2) \mathrm{S}_{\mathbf{H}}^{1} \mathrm{SU}(2) \subset \mathrm{U}(2) \mathrm{SO}(5), \mathrm{SO}(5) \mathrm{U}(2), n=4$ \\
where $\mathrm{S}_{\mathbf{H}}^{1}=\left\{\operatorname{diag}\left(z^{l_{1}}, z^{l_{1}}, 1, z^{l_{2}}, z^{l_{2}}\right)\right\}$ and ${ }_{1}=2, l_{2}=1$ or $l_{1}=1=l_{2}$. \\
\hline
\end{tabular}

Table 9: Possibilities for $K_{+}$

\begin{tabular}{|c|c|l|l|}
\hline Factors & Subcase & Group & Conditions \\
\hline 2 & $2 \mathrm{a}$ & $\operatorname{Spin}(2) \operatorname{Spin}(6)$ & $n=4$ \\
\hline 2 & $2 \mathrm{~b}$ & $\operatorname{Spin}(6) \operatorname{Spin}(6)$ & $n=6$ \\
\hline 2 & $2 \mathrm{c}$ & $\operatorname{Spin}(2) \operatorname{Spin}(5)$ & $n=3$ \\
\hline 2 & $2 \mathrm{~d}$ & $\operatorname{Spin}(5) \operatorname{Spin}(6)$ & $n=5$ \\
\hline 2 & $2 \mathrm{e}$ & $\hat{\mathrm{U}}(n)$ & - \\
\hline 3 & $3 \mathrm{a}$ & $\hat{\mathrm{U}}(n-3) \operatorname{Spin}(6)$ & $n \geq 5$ \\
\hline 3 & $3 \mathrm{~b}$ & $\hat{\mathrm{U}}(n-2) \operatorname{Spin}(5)$ & $n \geq 4$ \\
\hline 3 & $3 \mathrm{c}$ & $\mathrm{Spin}(2) \hat{\mathrm{U}}(n-1)$ & - \\
\hline 4 & $4 \mathrm{a}$ & $\hat{\mathrm{U}}\left(n_{1}\right) \hat{\mathrm{U}}\left(n_{2}\right)$ & $n_{1}, n_{2} \geq 1, n_{1}+n_{2}=n$ \\
\hline
\end{tabular}

2a) We transfer the whole situation into $\mathrm{SO}(9)$ and have $\mathrm{K}_{+}=\mathrm{S}^{1} \mathrm{SO}(6)$ and $\mathrm{H}=\mathrm{S}^{1} \mathrm{SU}(3)$, where we can write $\mathrm{S}^{1}=\left\{\operatorname{diag}\left(1, z^{l}, z^{k}, \ldots, z^{k}\right) \mid z \in \mathrm{S}^{1}\right\}$. But $\mathrm{K}_{+} / \mathrm{H}$ being a projective space implies $\mathrm{SO}(6) \cap \mathrm{H}=\mathrm{SU}(3)$, which can be written as

$$
\mathrm{SO}(6) \cap \mathrm{H}=\left\{\operatorname{diag}\left(1, z^{l}, z^{k}, z^{k}, z^{k}\right) \cdot A \mid A \in \mathrm{SU}(3), z^{l}=1\right\}
$$

so that $z^{3 k}=1$ whenever $z^{l}=1$, which implies that $l$ divides $3 k$. But $\operatorname{gcd}(k, l)=1$, and by reparametrisation we can assume $l=1,3$. We now divide cases by $\left(\mathrm{H}_{-}\right)_{0}$ :

- If $\left(\mathrm{H}_{-}\right)_{0}=\mathrm{SU}(3)$, we know $\mathrm{K}_{-} / \mathrm{H}_{-}$is $\mathrm{U}(2)$ or $\mathrm{SO}(3)$. But the normalizer $N(\mathrm{SU}(3))=\mathrm{SO}(3) \mathrm{S}^{1}$ does not contain $\mathrm{SU}(2)$, and 
$\mathrm{K}_{-} / \mathrm{H}_{-}=\mathrm{SO}(3)$ implies $k=0$, in which case we have that $\mathrm{H} \hookrightarrow \mathrm{SO}(9)$ is surjective on the fundamental group, a contradiction.

- If $\left(\mathrm{H}_{-}\right)_{0}=\mathrm{S}^{1}$, we have $N\left(\mathrm{~S}^{1}\right)=\mathrm{S}^{1} \mathrm{U}(3)$ for $k \neq 1$, so we will not get a primitive example in this case. If $k=1$, we have $N\left(\mathrm{~S}^{1}\right)=\mathrm{U}(4)$, but both $\mathrm{K}_{ \pm}$are contained in $\mathrm{SO}(8)$, so again no primitive example can be found here.

- If $\mathrm{H}_{-}$is finite, we have $\mathrm{K}_{-}=\mathrm{S}^{1} \mathrm{SU}(4)$ or $\mathrm{K}_{-}=\mathrm{S}^{1} \mathrm{SO}(6)$, but in both cases $\mathrm{K}_{ \pm} \subset \mathrm{SO}(8)$ again, a contradiction to primitivity.

2b) Again, by virtue of $-1 \in \mathrm{K}_{+} \backslash \mathrm{H}$ we look at the situation in $\mathrm{SO}(13)$. We have $\mathrm{SO}(6) \subset \mathrm{H}_{+}$, and therefore $l_{ \pm}>1$, so that all of $\mathrm{K}_{ \pm}, \mathrm{H}$ are connected. But $\mathrm{SO}(6) \subset \mathrm{H}$ implies that $\mathrm{SO}(13) / \mathrm{H}$ is simply connected, a contradiction, because $\operatorname{Spin}(13) /\left(\pi^{-1}(\mathbf{H})\right)_{0}$ is a nontrivial cover.

2c) We transfer the discussion to $\mathrm{SO}(7)$. Then we have $\mathrm{H}=\mathrm{S}^{1} \mathrm{SU}(2)$, where $\mathrm{S}^{1}=\left\{\operatorname{diag}\left(z^{l}, z^{k}, z^{k}, 1\right) \mid z \in \mathrm{S}^{1}\right\}$ (where $\left.(k, l)=1\right)$ and $\mathrm{SU}(2)$ is given accordingly. We have $\mathrm{SO}(5) / \mathrm{SU}(2)=\mathbb{R} P^{7}$ so that $\mathrm{SO}(5) \cap \mathrm{H}=\mathrm{SU}(2)$, so that $z^{2 k}=1$ whenever $z^{l}=1$, implying that $l$ divides $2 k$. But $(k, l)=1$, so that $l$ divides 2 , and therefore $l= \pm 1, \pm 2$. But the image of $\pi_{1}\left(\mathrm{~S}^{1}\right) \rightarrow \pi_{1}(\mathrm{SO}(7))$ is given by $l \bmod 2 \in \mathbb{Z}_{2}$, implying $l=2$ (if $l= \pm 1, \mathrm{SO}(7) / \mathrm{H}$ is simply connected, a contradiction as before) and $k$ is odd. We assume $l=2$ (possibly reparametrizing $\mathrm{S}^{1}$ ) and divide cases by $\left(\mathbf{H}_{-}\right)_{0}$ :

- If $\mathrm{H}_{-}=\mathrm{H}$, we have $\mathrm{K}_{-} \subset N(\mathrm{H})$ and $N(\mathrm{H})_{0}=\mathrm{T}^{2} \mathrm{SU}(2) \subset \mathrm{K}_{+}$, a contradiction to primitivity.

- If $\left(\mathrm{H}_{-}\right)_{0}=\mathrm{S}^{1}$, we have $N\left(\mathrm{~S}^{1}\right)=\mathrm{T}^{2} \mathrm{SU}(2) \subset \mathrm{K}_{+}$as before, a contradiction.

- If $\left(\mathrm{H}_{-}\right)_{0}=\mathrm{SU}(2)$, we have $N(\mathrm{SU}(2))=\mathrm{S}^{1} \mathrm{SU}(2) \mathrm{SO}(3)$. Since $\mathrm{H} / \mathrm{H}_{-}=\mathrm{S}^{1}$, we have $\mathrm{K}_{-} / \mathrm{H}_{-}=\mathrm{U}(2)$ or $\mathrm{SO}(3)$. But the only $\mathrm{SU}(2)$ in $N\left(\mathrm{H}_{-}\right)$is also in $\mathrm{H}_{-}$, and the $\mathrm{SO}(3)$-factor does not contain $\mathrm{S}^{1}$, so there's no example in this case either.

- If $\mathrm{H}_{-}$is finite, we have either $\mathrm{K}_{-} \simeq \mathrm{U}(3)$ or $\mathrm{K}_{-} \simeq \mathrm{SO}(2) \mathrm{SO}(5)$, where the second case of course does not give a primitive manifold. $\mathrm{SU}(3)$ is determined by $\mathrm{H}$, and the center of $\mathrm{K}_{-}$is determined by $\mathrm{H}$ up to the first coordinate. It is either given by $\{\operatorname{diag}(z, z, z)\}$ or $\{\operatorname{diag}(\bar{z}, z, z\}$. But conjugation of the first coordinate fixes both $\mathrm{K}_{+}$and $\mathrm{H}$, so we can assume $\mathrm{K}_{-}$is the standard upper 
left block. Since $\mathrm{U}(3) \hookrightarrow \mathrm{SO}(7)$ is surjective on the fundamental group, $\pi^{-1}(\mathrm{U}(3))$ is connected and contains -1 , so that $\mathrm{K}_{-} / \mathrm{H}$ is a projective space. Now $\mathrm{SU}(3) \cap \mathrm{H}=\mathrm{SU}(2)$, which implies $k=1$ or $k=-3$ (note that $\mathrm{SU}(3) \cap \mathrm{H} / \mathrm{SU}(2)=\mathbb{Z}_{2}$ and that $z= \pm 1$ gives lements of $\mathrm{SU}(2))$. Those are obviously two primitive examples.

2d) This follows the exact same reasoning of case $2 \mathrm{~b}$ above, and does not give a primitive example.

2e) Since $-1 \in \mathrm{K}_{+}$, again we transfer the situation to $\mathrm{SO}(2 n+1)$. We have $\mathrm{SU}(n) \not \subset \mathrm{H}_{+}$, since otherwise $\mathrm{SU}(n+1) \subset \mathrm{K}_{-}$, but there is no embedding $\mathrm{SU}(n+1) \hookrightarrow \operatorname{Spin}(2 n+1)$. So we know $\mathrm{H}=\mathrm{S}_{k}^{1} \mathrm{SU}(n-1)$ where

$$
\mathrm{S}_{k}^{1}=\left\{\left(\operatorname{diag}\left(1, z^{(k+2)(n-1)}, z^{-k}, \ldots, z^{-k}\right) \mid z \in \mathrm{S}^{1}\right\}\right.
$$

and $k$ is odd (see section B.2.3 on page 68). This implies $N(\mathrm{H})_{0}=$ $\mathrm{T}^{2} \mathrm{SU}(n-1) \subset \mathrm{K}_{+}$, so that $\mathrm{H} \neq \mathrm{H}_{-}$and $l_{-}>1$. Also, $N(\mathrm{SU}(n-1))_{0}=$ $\mathrm{SO}(3) \mathrm{U}(n-1)$, but the $\mathrm{SO}(3)$-factor does not contain $\mathrm{S}_{k}^{1}$, so in fact $l_{-}>2$. Looking at both normalizers and checking against the list of effective transitive sphere actions ( 16 on page 65 ) we also see $l_{-}>3$. For $n \geq 5$, this implies $\mathrm{K}_{-} \simeq \mathrm{U}(n)$, but it is already determined by $\mathrm{S}_{k}^{1}$ (note that $k$ cannot be -2 ), so that $\mathrm{K}_{ \pm} \subset \mathrm{SO}(2 n)$ and no primitive example arises in this case.

If $n \leq 4$, there are two additional possibilities for $\mathrm{K}_{-}$, coming from the accidental isomorphisms $\mathrm{Sp}(2) \simeq \operatorname{Spin}(5)$ and $\mathrm{SU}(4) \simeq \operatorname{Spin}(6)$. They have already been considered in $2 \mathrm{c}$ and $2 \mathrm{a}$ respectively.

3a) We have $-1 \in \mathrm{K}_{+}$, so we transfer the situation to $\mathrm{SO}(2 n+1)$ again. We cannot have $\mathrm{SO}(6) \subset \mathrm{H}$, for that would imply that $\mathrm{SO}(2 n+1) / \mathrm{H}$ is simply connected. But then $\mathrm{SU}(n-3) \subset \mathrm{H}_{+}$, because $\mathrm{SO}(6) \times \mathrm{SU}(n-3)$ can not act transitively almost effectively on a projective space. Now also $\mathrm{SO}(5) \not \subset \mathrm{H}$ for the same reasons as above, so we actually have $\mathrm{H}=\mathrm{SU}(3) \mathrm{S}^{1} \mathrm{SU}(n-3)$. Since $\mathrm{K}_{-}$can not contain $\mathrm{SU}(3) \mathrm{SU}(n-2)$ (which has no embedding into $\mathrm{SO}(2 n+1)$ ), we have that $\mathrm{SU}(n-3)$ is contained in the isotropy group of an almost effective tansitive action on a sphere of a group that does not contain $\mathrm{SU}(n-2)$ as a transitively acting factor. This implies $n=5$ or $n=6$.

If $n=5$, we have $\mathrm{K}_{-}=\mathrm{SU}(3) \mathrm{S}^{1} \mathrm{SO}(5)$ (note that $\mathrm{SO}(5) / \mathrm{SU}(2)$ is a projective space and $\mathrm{Spin}(5)=\mathrm{Sp}(2))$, but $\mathrm{SO}(5)$ is determined by 
$\mathrm{SU}(3)$ and $\mathrm{SU}(2)$, and it's easy to see $\mathrm{K}_{ \pm} \subset \mathrm{SO}(6) \mathrm{SO}(5)$, so that this is not primitive.

If $n=6$, we have $\mathrm{H}=\mathrm{SU}(3) \mathrm{S}^{1} \mathrm{SU}(3)$ and the above reasoning implies $\mathrm{K}_{-}=\mathrm{SU}(3) \mathrm{S}^{1} \mathrm{SO}(6)$, but again $\mathrm{K}_{ \pm} \subset \mathrm{SO}(6) \mathrm{SO}(6)$.

3b) Again, we look at the image of the diagram in $\mathrm{SO}(2 n+1)$ and have $\mathrm{K}_{+}=\mathrm{U}(n-2) \mathrm{SO}(5)$. We have $\mathrm{SO}(5) \not \subset \mathrm{H}$ (because $\mathrm{G} / \mathrm{H}$ is not simply connected), so $\mathrm{SU}(n-2) \subset \mathrm{H}_{-}$, which implies $\mathrm{H}=\mathrm{SU}(n-2) \tilde{\mathrm{S}}^{1} \mathrm{SU}(2)$. Now $\operatorname{SU}(n-1) \mathrm{SU}(2) \subset \mathrm{K}_{-}$is a contradiction (we can see there is no such embedding into $\mathrm{SO}(2 n+1)$ by looking at the dimensions of representations), and since $\mathrm{SU}(n-2) \subset \mathrm{H}_{-}$we have $n=4$ or $n=5$. But $n=5$ implies $\mathrm{K}_{-} \simeq \mathrm{SU}(2) \mathrm{S}^{1} \mathrm{SO}(6)$, which does not give a primitive example by $3 \mathrm{a})$.

So we are left with $n=4$, and $\mathrm{K}_{-}=\mathrm{SU}(2) \mathrm{S}^{1} \mathrm{SO}(5)$. We know $\tilde{\mathrm{S}}^{1} \subset \mathrm{H}$ commutes with both $\mathrm{SU}(2)$-factors, so it is of the form

$$
\tilde{\mathbf{S}}^{1}=\left\{\operatorname{diag}\left(z^{l_{1}}, z^{l_{1}}, 1, z^{l_{2}}, z^{l_{2}}\right) \mid z \in \mathbf{S}^{1}\right\}
$$

for some $l_{1}, l_{2} \in \mathbb{Z}$ with $\left(l_{1}, l_{2}\right)=1$. Since complex conjugation on $\mathrm{U}(2)$ can be realized by a matrix in $\mathrm{SO}(4)$, we can reconjugate the diagram so that $l_{1}, l_{2} \geq 0$, without changing $\mathrm{K}_{ \pm}$. Now since $\mathrm{K}_{+} / \mathrm{H}$ is a projective space, we know $\mathrm{SO}(5) \cap \mathrm{H}=\mathrm{SU}(2)$, so we know that $z^{l_{1}}= \pm 1$ for all $z$ that satisfy $z^{l_{2}}=1$, i.e. $l_{2} \mid 2 l_{1}$. But $l_{1}, l_{2}$ are coprime, so $l_{2} \mid 2$, so $l_{2}=1$ or $l_{2}=2$. By symmetry reasons $\left(\mathrm{K}_{-}\right.$is a conjugate of $\mathrm{K}_{+}$by just exchanging the first 4 coordinates with the last 4 ), also $l_{1}=1$ or $l_{1}=2$. Exchanging $\mathrm{K}_{+}$and $\mathrm{K}_{-}$does not change the manifold by section 1.2 on page 13 , we can assume $l_{1} \geq l_{2}$ and are left with 2 examples. Since exchanging the first 4 coordinates with the last 4 is not in in the unity component of the normalizer of $\mathrm{H}$ (all automorphisms in $N(\mathrm{H})_{0}$ are inner), those 2 examples are primitive.

3c) Since $-1 \in \mathrm{K}_{+}$, we translate the situation to $\mathrm{SO}(2 n+1)$. First assume $\mathrm{SU}(n-1) \subset \mathrm{H}_{+}$. This implies $\mathrm{K}_{-} \simeq \mathrm{U}(n)$, so no primitive example arises. Note that $\mathrm{H} / \mathrm{H}_{-}$contains $\mathrm{S}^{1} \mathrm{SU}(n-1)$, which also excludes the cases $n=3$ and $\mathrm{K}_{-} \supset \mathrm{Sp}(2)$ and $n=4$ and $\mathrm{K}_{-} \supset \mathrm{SO}(6)$.

So now assume $\mathrm{H}=\mathrm{T}^{2} \mathrm{SU}(n-2)$. We have $\mathrm{S}^{1} \subset \mathrm{H} / \mathrm{H}_{-}$, so either $\mathrm{H}_{/} \mathrm{H}_{-}=\mathrm{S}^{1}$ or $\mathrm{H} / \mathrm{H}_{-}=\mathrm{S}^{1} \mathrm{SU}(n-2)$. The latter case just implies $\mathrm{K}_{-} \simeq \mathrm{K}_{+}$, which does not give a primitive example for the following reason: Note that if all of $\mathrm{H}, \mathrm{K}_{ \pm}$have a trivial one-dimensional subrepresentation from their inclusion into $\mathrm{SO}(2 n+1)$, these are neccessarily 
the same, so $\mathrm{K}_{ \pm} \subset \mathrm{SO}(2 n)$. Otherwise $\mathrm{H}$ has three one-dimensional trivial subrepresentations, and then $\mathrm{SO}(3) \subset N(\mathrm{H})_{0}$, which includes the change of coordinate which allows us to assume $\mathrm{K}_{ \pm}$act trivial on the same one-dimensional subspace, and $\mathrm{K}_{ \pm} \subset \mathrm{SO}(2 n)$ as before.

So we know $\mathrm{H} / \mathrm{H}_{-}=\mathrm{S}^{1}$, and we divide cases by $l_{-}=3$ and $l_{-}=2$ :

- If $l_{-}=2$, then $\mathrm{K}_{-} / \mathrm{H}_{-}$is either $\mathrm{SO}(3)$ or $\mathrm{SU}(2)$. The first case would imply that $\operatorname{Spin}(3) \subset\left(\pi^{-1}\left(\mathrm{~K}_{-}\right)\right)_{0}$ acts on the sphere $\mathrm{S}^{l_{-}}$, but $-1 \in \operatorname{Spin}(3)$ has to acts trivial, a contradiction. In the second case $\mathrm{K}_{-}$has a trivial 1-dimensional subrepresentation as a subgroup of $\mathrm{SO}(2 n+1)$, which is necessarily given by $\mathbb{R} e_{1}$ (because we have $\mathrm{SU}(2) \subset N\left(\mathrm{~S}^{1} \mathrm{SU}(n-2)\right)$, so this is not a primitive example.

- If $l_{-}=3$, we have $\mathrm{K}_{-} / \mathrm{H}_{-}=\mathrm{U}(2)$, so that $\mathrm{K}_{-}=\mathrm{T}^{2} \mathrm{SU}(2) \mathrm{SU}(n-2)$. First assume that $\mathrm{H}$ as a subgroup of $\mathrm{SO}(2 n+1)$ has exactly one 1-dimensional trivial subrepresentation. This is neccessarily $\mathbb{R} e_{1}$, and the same is true for $\mathrm{K}_{-}$, which also has exactly one $1-$ dimensional trivial subrepresentation on which $\mathrm{H}$ need to act trivially, too. The other possibility is that $\mathrm{H}$ has three 1-dimensional trivial subrepresentations, but then $N(\mathrm{H})_{0} \supset \mathrm{SO}(3) \mathrm{SU}(n-2)$ and so by conjugation in $N(\mathrm{H})_{0}$ we can assume that $\mathrm{K}_{-}$acts trivially on $\mathbb{R} e_{1}$, again a contradiction to primitivity.

4a) Just as in $4 a$ ) of case $\mathrm{G}=\mathrm{SO}(2 n+1)$, no primitive example can arise in this case. The proof carries over verbatim by transferring the situation to $\mathrm{SO}(2 n+1)$. 


\section{$8 \mathrm{G}=\mathrm{Sp}(n), n \geq 2$}

For a general description of the classification procedure, see section 3 on page 20 .

We claim that for $n>1$ the simply connected primitive cohomogeneity one $\operatorname{Sp}(n)$-manifolds with positive euler characteristic are given by table 10 . Note that we will not treat $\operatorname{Sp}(2) \simeq \operatorname{Spin}(5)$ the way we deal with the spin groups as described in section 3.1 on page 20, but just follow the procedure for the non-Spin groups.

Table 10: $\operatorname{Sp}(n)$-cohomogeneity one manifolds for $n>1$

\begin{tabular}{|c|c|}
\hline $\begin{array}{r}\operatorname{Sp}(n-2) \Delta \mathrm{Sp}(1) \subset \mathrm{Sp}(n-2) \mathrm{S}( \\
\text { where } \Delta \mathrm{Sp}(1) \text { is the diagonal } \mathrm{Sp}(1 \\
\text { and } \mathrm{SO}(2) \text { is the standard } \mathrm{S}\end{array}$ & $\begin{array}{l}\mathrm{O}(2) \Delta \mathrm{Sp}(1), \operatorname{Sp}(n-1) \mathrm{Sp}(1) \\
\mathrm{O}(2) \text { in the upper left } \mathrm{Sp}(2) \text {-block } \\
\end{array}$ \\
\hline $\begin{array}{r}\mathbb{Z}_{2} \Delta \mathrm{Sp}(1) \subset \mathrm{SO}(2) \Delta \mathrm{Sp}(1) \\
\text { where } \mathbb{Z}_{2} \text { is generatec } \\
\mathrm{SO}(2) \text { and } \Delta \mathrm{Sp}(1)\end{array}$ & $\begin{array}{l}\mathbb{Z}_{2} \operatorname{Sp}(1) \operatorname{Sp}(1), n=2 \\
\text { by }\left(\begin{array}{ll}0 & 1 \\
1 & 0\end{array}\right) \text { and } \\
\text { are as above }\end{array}$ \\
\hline $\begin{array}{r}\quad \mathrm{S}^{1} \mathrm{Sp}(n-2) \subset \mathrm{U}(2) \mathrm{Sp}( \\
\text { where } \mathrm{S}^{1}=\left\{\operatorname{diag}\left(z, \bar{z}^{2}, 1, \ldots,\right.\right.\end{array}$ & $\begin{array}{l}n-2), S^{1} \operatorname{Sp}(n-1) \\
1)\} \text { or }\{\operatorname{diag}(z, 1, \ldots, 1)\}\end{array}$ \\
\hline $\mathrm{U}\left(n_{1}\right) \operatorname{Sp}\left(n_{2}-1\right) \subset \mathrm{U}\left(n_{1}+1\right) \operatorname{Sp}\left(n_{2}\right.$ & $-1), \mathrm{U}\left(n_{1}\right) \operatorname{Sp}\left(n_{2}\right), n_{1}+n_{2}=n$ \\
\hline $\begin{array}{r}\mathrm{S}_{\mathrm{H}}^{1} \mathrm{Sp}(n-2) \subset \mathrm{Sp}(1) \mathrm{Sp} \\
\text { where } \mathrm{S}_{\mathrm{H}}^{1}=\left\{\operatorname{diag}\left(z, z^{3}, 1, \ldots, 1\right)\right\} \text { and } \\
\text { given by the irreducib }\end{array}$ & $\begin{array}{l}(n-2), \mathrm{S}^{1} \mathrm{Sp}(n-1) \\
\mathrm{Sp}(1) \hookrightarrow \mathrm{Sp}(2) \text { (upper left block) } \\
\mathrm{le} \mathrm{SO}(3) \hookrightarrow \mathrm{SO}(5)\end{array}$ \\
\hline $\begin{array}{r}\mathrm{T}_{\mathrm{H}}^{2} \mathrm{Sp}(n-3) \subset \mathrm{S}^{1} \mathrm{SO}^{\prime}(3) \mathrm{S} \\
\text { where } \mathrm{S}^{1}=\{\operatorname{diag}(z, z, z, 1, \ldots, 1)\} \\
\text { and } \mathrm{SO}^{\prime}(3) \text { is the conjugation } \\
\mathrm{SO}(3) \text {-block by }\end{array}$ & $\begin{array}{l}\mathrm{p}(n-3), \mathrm{T}^{2} \mathrm{Sp}(n-2) \\
\mathrm{T}_{\mathrm{H}}^{2}=\{\operatorname{diag}(1, z, \bar{z}, 1, \ldots, 1)\} \cdot \mathrm{S}^{1} \\
\text { of the standard upper left } \\
\left(\begin{array}{ccc}1 & 0 & 0 \\
0 & 1 & i \\
0 & 1 & -i\end{array}\right)\end{array}$ \\
\hline
\end{tabular}


The possibilities for $\mathrm{K}_{+}$are given by table 11 .

Table 11: Possibilities for $K_{+}$

\begin{tabular}{|c|l|l|}
\hline Factors & Group & Conditions \\
\hline 2 & $\mathrm{U}(n)$ & - \\
\hline 2 & $\mathrm{Sp}\left(n_{1}\right) \operatorname{Sp}\left(n_{2}\right)$ & $n_{1}+n_{2}=n$ \\
\hline 2 & $\mathrm{~S}^{1} \mathrm{Sp}(n-1)$ & - \\
\hline 2 & $\mathrm{~T}^{2}$ & $n=2$ \\
\hline 3 & $\mathrm{U}\left(n_{1}\right) \operatorname{Sp}\left(n_{2}\right)$ & $n_{1}>1, n_{1}+n_{2}=n$ \\
\hline 3 & $\mathrm{~S}^{1} \mathrm{U}(n-1)$ & $n \geq 3$ \\
\hline 3 & $\mathrm{~S}^{1} \mathrm{Sp}\left(n_{1}\right) \operatorname{Sp}\left(n_{2}\right)$ & $n_{1}+n_{2}+1=n$ \\
\hline 3 & $\mathrm{Sp}(1) \operatorname{Sp}\left(n_{1}\right) \operatorname{Sp}\left(n_{2}\right)$ & $n_{1}+n_{2}+1=n$ \\
\hline 3 & $\mathrm{~T}^{2} \operatorname{Sp}(n-2)$ & $n \geq 3$ \\
\hline 4 & $\mathrm{U}\left(n_{1}\right) \mathrm{U}\left(n_{2}\right)$ & $n_{1}, n_{2}>1, n_{1}+n_{2}=n$ \\
\hline 4 & $\mathrm{Sp}(1) \operatorname{Sp}\left(n_{1}\right) \operatorname{Sp}(1) \operatorname{Sp}\left(n_{2}\right)$ & $n_{1}, n_{2} \geq 1, n \geq 4$ \\
\hline 4 & $\mathrm{Sp}(1) \operatorname{Sp}\left(n_{1}\right) \mathrm{S}^{1} \mathrm{Sp}\left(n_{2}\right)$ & $n_{1}, n_{2} \geq 1, n \geq 4$ \\
\hline 4 & $\mathrm{~T}^{2} \operatorname{Sp}\left(n_{1}\right) \operatorname{Sp}\left(n_{2}\right)$ & $n_{1}, n_{2} \geq 1, n \geq 4$ \\
\hline 4 & $\operatorname{Sp}(1) \operatorname{Sp}\left(n_{1}\right) \mathrm{U}\left(n_{2}\right)$ & $n_{1} \geq 1, n_{2} \geq 2, n \geq 4$ \\
\hline 4 & $\mathrm{~S}^{1} \operatorname{Sp}\left(n_{1}\right) \mathrm{U}\left(n_{2}\right)$ & $n_{1} \geq 1, n_{2} \geq 2, n \geq 4$ \\
\hline
\end{tabular}

2a) If $n \neq 2, \operatorname{SU}(n)$ cannot be contained in the kernel, for that would mean $\mathrm{SU}(n) \subset \mathrm{K}_{-} \cap \mathrm{H}$ would act almost effectively on $\mathrm{K}_{-} / \mathrm{H}$, so that $\mathrm{SU}(n+1) \subset \mathrm{K}_{-}$. But there's no embedding of $\mathrm{SU}(n+1) \hookrightarrow \mathrm{Sp}(n)$ save the case $n=1$, which is not included here. This is easily seen by looking at the dimensions of representations.

So assume $\mathrm{SU}(n) \not \subset \mathrm{H}_{+}$. Now we know $\mathrm{H}$ is given by $\mathrm{S}_{k}^{1} \mathrm{SU}(n-1)$ where $\mathrm{S}_{k}^{1}$ is given by

$$
\mathrm{S}_{k}^{1}=\left\{\operatorname{diag}\left(z^{(k+1)(n-1)}, z^{-k}, \ldots, z^{-k}\right) \mid z \in \mathrm{S}^{1}\right\}
$$


The unity component of the normalizer of $\mathrm{H}$ in $\mathrm{Sp}(n)$ is given by either $\mathrm{Sp}(1) \mathrm{U}(n-1)$ (for $k=-1$ ) or $\mathrm{S}^{1} \mathrm{~S}_{k}^{1} \mathrm{SU}(n-1)$ (for $k \neq-1$ ), where $\mathrm{S}^{1}$ is the standard one in the left upper $\operatorname{Sp}(1)$ block. Let's first assume $\mathrm{K}_{-} \neq$ $\mathrm{U}(n)$. For $k=-1$, we can have $\mathrm{K}_{-}=\operatorname{Sp}(1) \mathrm{U}(n-1)$, giving a primitve manifold. There's also the case $\mathrm{K}_{-}=\mathrm{S}^{1} \mathrm{H}$, where by conjugation in $N(\mathrm{H})_{0}$ we can assume $\mathrm{S}^{1} \subset \mathrm{K}_{-}$is standard. But this is not primitive, for $\mathrm{K}_{-} \subset \mathrm{K}_{+}$. For $k \neq-1$ there's only the case $\mathrm{S}^{1} \mathrm{H}$ where $\mathrm{S}^{1}$ is standard, which isn't primive.

If $\mathrm{K}_{-} \simeq \mathrm{U}(n)$, we can determine the embedding via the center as in case $2 \mathrm{a}$ ) of section 9 on page 58 , which also leads to $\mathrm{K}_{-}=\overline{\mathrm{U}}(n)$ as the only primitive possibility, which only works in the cases $k=-1$ or $k=$ 0 . But $\hat{\mathrm{U}}(n)=\sigma(\mathrm{U}(n))$ where $\sigma$ is conjugation $\operatorname{by} \operatorname{diag}(j, 1, \ldots, 1) \in$ $N(\mathrm{H})_{0}$ for $k=-1,0$, so no primitive manifold arises.

Lastly assume $\mathrm{SU}(n) \subset \mathrm{H}_{+}$which implies $n=2$ as said above. Since now $\mathrm{H}_{0}=\mathrm{SU}(2)$ and there's no embedding of $\mathrm{SU}(3)$ into $\mathrm{Sp}(2)$, we have $\left(\mathrm{K}_{-}\right)_{0} \simeq \mathrm{Sp}(1) \mathrm{Sp}(1)$. We reconjugate the diagram so that $\left(\mathrm{K}_{-}\right)_{0}$ is standard, and $(\mathrm{H})_{0}=\Delta \mathrm{Sp}(1)$ is diagonal. This already determines the maximal subgroup of maximal rank $\mathrm{K}_{+}=\mathrm{S}^{1} \Delta \mathrm{Sp}(1)$. If all groups are connected, this gives a primitive manifold. The normalizer of $\left(\mathrm{K}_{-}\right)_{0}$ in $\operatorname{Sp}(2)$ is $\mathbb{Z}_{2} \operatorname{Sp}(1) \operatorname{Sp}(1)$, where the outer automorphism in $\mathbb{Z}_{2}$ switches the two $\mathrm{Sp}(1)$-factors. This gives $H=\mathbb{Z}_{2} \Delta \mathrm{Sp}(1)$ and $\mathrm{K}_{+}$as before, another primitive manifold.

2b) First assume $\operatorname{Sp}\left(n_{2}\right) \subset \mathrm{H}_{+}$. If $n_{1}>2$, so that $\mathrm{H}=\operatorname{Sp}\left(n_{1}-1\right) \operatorname{Sp}\left(n_{2}\right)$ and we know from primitivity that $\operatorname{Sp}\left(n_{2}\right) \subset \mathrm{H} / \mathrm{H}_{-}$and therefore $\mathrm{K}_{-}=$ $\operatorname{Sp}\left(n_{1}-1\right) \operatorname{Sp}\left(n_{2}+1\right)$. Note that in the case $n_{2}=2$ we have $\mathbf{H} \simeq$ $\mathrm{Sp}(n-3) \operatorname{Spin}(5)$, but we cannot have $\mathrm{H} \simeq \mathrm{Sp}(n-3) \operatorname{Spin}(6)$, because that would be a maximal rank subgroup, but not isomorphic to one of the groups we have given in table 15 on page 65 . In the case $n_{2}=1$ we have $\mathrm{H}=\mathrm{Sp}(n-2) \mathrm{Sp}(1)$ so that we can have $\mathrm{K}_{-}=\mathrm{Sp}(n-2) \operatorname{Spin}(4)=\operatorname{Sp}(n-$ 2) $\mathrm{Sp}(1) \mathrm{Sp}(1)$. But the standard representation of $\operatorname{Sp}(n)$ restricted to $\mathrm{H}$ factors into 3 nonequivalent irreducible representations $\mathbb{H}^{n-2} \oplus \mathbb{H} \oplus \mathbb{H}$ where the last factor is acted on trivially. This already determines $\mathrm{K}_{-}$, which is standard, which would imply $\mathrm{Sp}(1) \subset \mathrm{H}_{-} \cap \mathrm{H}_{+}$, a contradiction.

If $n_{1} \leq 2$ the possibilities given above arise as well, but we need to note that because of $\operatorname{corank}(\mathrm{H})=1$ by corollary 1.7 on page 15 we cannot have $\mathrm{K}_{+} / \mathrm{H}_{+} \simeq \mathrm{SO}(3)$ or $\mathrm{SO}(5)$.

Of course, if $\operatorname{Sp}\left(n_{1}\right) \subset \mathrm{H}_{+}$we may just exchange $\operatorname{Sp}\left(n_{1}\right)$ with $\operatorname{Sp}\left(n_{2}\right)$, so now we assume that none of both is contained in $\mathrm{H}_{+}$, which amounts to either $n_{1}=1$ or $n_{2}=1$ (just assume the latter) and $\mathrm{H}=\operatorname{Sp}(n-$ 
2) $\Delta \mathrm{Sp}(1)$. The identity component of the normalizer of $\mathrm{H}$ in $\mathrm{G}$ is given by $N(\mathrm{H})_{0}=\mathrm{S}^{1} \Delta \mathrm{Sp}(1) \mathrm{Sp}(n-2)$, where $\mathrm{S}^{1}$ is given by $\mathrm{SO}(2) \hookrightarrow \mathrm{Sp}(2)$. We can go through the possibilities for $\mathbf{K}_{-}$by studying the isotropy representation. Two of the possibilities amount to $\mathrm{K}_{-} \simeq \mathrm{K}_{+}$, one of which is obviously not primitive $\left(\mathrm{K}_{-}=\mathrm{K}_{+}\right)$, and the other one where $\mathrm{K}_{-}$is obtained from $\mathrm{K}_{+}$by exchanging the first two coordinates. This is given by conjugation with the matrix having

$$
\left(\begin{array}{cc}
0 & -1 \\
1 & 0
\end{array}\right)
$$

as the upper left 2x2-block, and extended by the identity to the other coordinates. This is in $N(\mathrm{H})_{0}$ though, so no new primitive manifold arises. We can also have $\mathrm{K}_{-}=\mathrm{Sp}(1) \mathrm{Sp}(1) \mathrm{Sp}(n-2)$, where both possible cases (the additional $\mathrm{Sp}(1)$ can be in the diagonal or offdiagonal in the upper right block of $\mathrm{Sp}(2)$ ) are conjugate (in $\mathrm{Sp}(2)$ this conjugation is given by

$$
\frac{1}{\sqrt{2}}\left(\begin{array}{cc}
1 & 1 \\
-1 & 1
\end{array}\right)
$$

But this is in $N(\mathrm{H})_{0}$ as well, so again no primitive example arises. The last case is $\mathrm{K}_{-}=\mathrm{S}^{1} \Delta \mathrm{Sp}(1) \mathrm{Sp}(n-2)$ where $\mathrm{S}^{1}$ is given by the standard embedding $\mathrm{SO}(2) \hookrightarrow \mathrm{Sp}(2)$, i.e. $\mathrm{K}_{-} \simeq \mathrm{U}(2) \mathrm{Sp}(n-2)$, which is primitive for the same reasons as above. Note that we have $l_{-}=1$ here, so that components may occur. But the Normalizer of $\mathrm{K}_{+}$in $\mathrm{G}$ is $\mathrm{K}_{+}$itself save the case $n=2$, which was already treated in 2 a.

2c) We cannot have $\mathrm{K}_{+} / \mathrm{H}_{+}=\mathrm{S}^{1}$, since that would imply $\mathrm{Sp}(n-1) \subset \mathrm{H}_{+}$ and therefore $\mathrm{K}_{-}=\mathrm{Sp}(n)$.

The almost effective actions of $\mathrm{S}^{1} \mathrm{Sp}(n-1)$ on spheres are given by $(z, A) \cdot v=A v z^{l}$ with isotropy group $\mathrm{Sp}(n-2) \Delta \mathrm{S}_{l}^{1}$, where $\Delta \mathrm{S}_{l}^{1}$ is given by

$$
\mathrm{S}_{l}^{1}:=\left\{\operatorname{diag}\left(z, z^{l}, 1, \ldots, 1\right) \mid z \in \mathrm{S}^{1}\right\}
$$

First assume $\mathrm{Sp}(n-2) \not \subset \mathrm{H}_{-}$. If $\Delta \mathrm{S}_{l}^{1} \subset \mathrm{H}_{-}$, we have $\mathrm{K}_{-}=\mathrm{S}_{l}^{1} \mathrm{Sp}(n-1)$ where $S_{l}^{1}$ normalizes the $\operatorname{Sp}(n-1)$ factor. That is only possible for $l=0$ and $\mathrm{K}_{-}=\mathrm{K}_{+}$. If $\Delta \mathrm{S}_{l}^{1} \not \subset \mathrm{H}_{-}$, then we have $\mathrm{K}_{-} \simeq \mathrm{K}_{+}$with only two 
choices for $\mathrm{K}_{-}$. One is $\mathrm{K}_{-}=\mathrm{K}_{+}$, and the other is $\mathrm{K}_{-}=\sigma\left(\mathrm{K}_{+}\right)$, where $\sigma$ is the coordinate change which exchanges the first 2 coordinates. But this implies $l=1$, where we have $N(\mathrm{H})_{0}=\mathrm{U}(2) \operatorname{Sp}(n-2)$, which includes $\sigma$, so this manifold is not primitive as well.

From now on we assume $\mathrm{Sp}(n-2) \subset \mathrm{H}_{-}$, that is, $\mathrm{H} / \mathrm{H}_{-}=\mathrm{S}^{1}$. First also assume $\mathrm{K}_{-} / \mathrm{H}_{-}=\mathrm{S}^{2}$, which implies $\mathrm{K}_{-}=\mathrm{Sp}(1) \mathrm{Sp}(n-2)$. Note that $\mathrm{Sp}(1)$ and $\Delta \mathrm{S}_{l}^{1}$ have to share a common central element of order 2. If $l$ is even, that element is $\operatorname{diag}(-1,1, \ldots, 1)$, which already implies that $\operatorname{Sp}(1)$ is standard in the upper left corner and $l=0$, obiously not a primitive manifold. If $n$ is odd, that element is $\operatorname{diag}(-1,-1,1, \ldots, 1)$, which is central in the upper left $\mathrm{Sp}(2)$ block, so we can look for embeddings $\mathrm{Sp}(1) \hookrightarrow \mathrm{Sp}(2)$ via embeddings $\mathrm{SO}(3) \hookrightarrow \mathrm{SO}(5)$. There are 2 of those: The standard embedding, which corresponds to $l=1$ by the induced weights for the isotropy representation of $\mathrm{S}^{1} \simeq \mathrm{SO}(2)$. This will not be primitive, because $N(\mathrm{H})_{0}$ contains $\mathrm{U}(2)$ in the upper left $2 \times 2$ block which acts transitively on the possible extensions $S_{1}^{1} \hookrightarrow \mathrm{Sp}(1)$, and $\mathrm{K}_{-}=\operatorname{Sp}(n-2) \Delta \operatorname{Sp}(1)$ is contained in $\operatorname{Sp}(1) \operatorname{Sp}(n-1)$ as well as $\mathrm{K}_{+}$. The other possible embedding is given via the representation on the traceless symmetric 3x3-matrices by conjugation (in representation terms, that's given by (4)) and corresponds to $l=3$. Note that this representation is irreducible, so that $\mathrm{K}_{-}$is not contained in $\operatorname{Sp}(1) \operatorname{Sp}(n-1)$, which is the maximal subgroup containing $\mathrm{K}_{+}$, so that the corresponding manifold is primitive.

The last case is $\mathrm{K}_{-} / \mathrm{H}_{-}=\mathrm{S}^{3}$. Since, as before, $\operatorname{Sp}(n-2) \subset \mathrm{H}_{-}$, we shift the discussion into the upper left $S p(2)$-block. The isotropy representation of $\Delta \mathrm{S}_{l}^{1}$ in $\mathrm{Sp}(2)$ has 4 summands of dimension 2 and at least one trivial of dimension one. Only the two off-diagonal summands (of weight $l \pm 1$ ) belong to groups not contained in $\operatorname{Sp}(1) S p(1)$, which would not lead to a primitive manifold, because $\mathrm{K}_{-} \subset \mathrm{K}_{+}$. The offdiagonal summands belong to the standard $\mathbf{S U}(2)$ in $\mathbf{S p}(2)$ as well as its conjugate by

$$
\left(\begin{array}{ll}
1 & 0 \\
0 & j
\end{array}\right)
$$

Note that this conjugation leaves $\mathrm{K}_{+}$invariant and just exchanges $\Delta \mathrm{S}_{l}^{1}$ with $\Delta \mathrm{S}_{-l}^{1}$ in $\mathrm{H}$, so we may just assume $\mathrm{K}_{-}=\mathrm{SU}(2) \Delta \mathrm{S}_{l}^{1}$, which leaves $l=0,-2$ (see section B.2 on page 66 ). In both cases, $\mathrm{K}_{ \pm}$are two differen maximal subgroups of maximal rank, so the manifolds are prim- 
itive.

2d) We have $\mathrm{H}_{0}=\mathrm{S}^{1}$, and by checking the isotropy representation as in the last paragraph of the previous case we can see $\left(\mathrm{K}_{-}\right)_{0}=\mathrm{U}(2)$ (note that again conjugation by $\operatorname{diag}(1, j)$ leaves $\mathrm{K}_{+}$invariant, and $\mathrm{H}$ additionally). Now $\mathrm{K}_{+} \subset \mathrm{K}_{-}$and no primitive manifold arises.

3a) First, suppost $\mathrm{Sp}\left(n_{2}\right) \subset \mathrm{H}_{+}$, so that $\mathrm{H}=\mathrm{S}_{k}^{1} \mathrm{SU}\left(n_{1}-1\right) \mathrm{Sp}\left(n_{2}\right)$ where

$$
\mathrm{S}_{k}^{1}=\left\{\operatorname{diag}(z^{(k+1)\left(n_{1}-1\right)}, \underbrace{z^{-k}, \ldots, z^{-k}}_{n_{1}-1}, \underbrace{1, \ldots, 1}_{n_{2}}) \mid z \in \mathrm{S}^{1}\right\}
$$

By primitivity $\operatorname{Sp}\left(n_{1}\right) \not \subset \mathrm{H}_{-}$, so that we must have $\mathrm{Sp}\left(n_{2}+1\right) \subset \mathrm{K}_{-}$, which is contained in the normalizer of $\mathrm{SU}\left(n_{1}-1\right)$, which implies $k=$ -1 if $n_{1} \neq 2$, which gives a primitive manifold. If $n_{1}=2$, we have $\mathrm{K}_{-} \simeq \mathrm{S}^{1} \mathrm{Sp}(n-1)$, which was treated in $2 \mathrm{c}$.

Now let $\mathrm{SU}\left(n_{1}\right) \subset \mathrm{H}_{+}$. Then $\mathrm{K}_{+}=\mathrm{S}^{1} \mathrm{SU}\left(n_{1}\right) \mathrm{Sp}\left(n_{2}\right)$ acts in the following way: For $(z, A) \in \mathrm{S}^{1} \mathrm{Sp}\left(n_{2}\right)$ we have $\mathrm{S}^{l_{+}} \ni v \mapsto A v z^{-k n_{1}}$. The isotropy group $\mathrm{H}$ is given by $\mathrm{H}=\mathrm{S}_{k}^{1} \mathrm{SU}\left(n_{1}\right) \mathrm{Sp}\left(n_{2}-1\right)$, where

$$
\mathrm{S}_{k}^{1}=\left\{\operatorname{diag}(\underbrace{z, \ldots, z}_{n_{1}}, z^{k n_{1}}, \underbrace{1, \ldots, 1}_{n_{2}-1}) \mid z \in \mathrm{S}^{1}\right\}
$$

By primitivity $\mathrm{SU}\left(n_{1}\right) \not \subset \mathrm{H}_{-}$. For $n_{1} \neq 2$ this implies $\mathrm{SU}\left(n_{1}+1\right) \subset \mathrm{K}_{-}$, which implies $k=0$ and $\mathrm{K}_{-}=\mathrm{U}\left(n_{1}+1\right) \mathrm{Sp}\left(n_{2}+1\right)$ or its conjugate by

$$
\operatorname{diag}(\underbrace{1, \ldots, 1}_{n_{1}}, j, \underbrace{1, \ldots, 1}_{n_{2}})
$$

But this conjugation leaves $\mathrm{H}$ invariant as well as $\mathrm{K}_{+}$, so both manifolds are equivalent. Note that we cannot have $S^{1} \subset \mathrm{H}_{-}$for we do have $\mathrm{S}^{1} \subset \mathrm{H}_{+}$. This is one primitive example.

If $n_{1}=2$, there is an additional posibbility: $\mathrm{K}_{-} \simeq \mathrm{S}^{1} \mathrm{Sp}(2) \operatorname{Sp}(n-3)$. We conjugate the whole diagram to make $\mathrm{K}_{-}$standard. Then $\mathrm{H}=$ $\mathrm{S}_{l}^{1} \operatorname{Sp}(1) \operatorname{Sp}(n-3)$ where

$$
\mathrm{S}_{l}^{1}=\left\{\operatorname{diag}\left(z, z^{l}, 1, \ldots, 1\right) \mid z \in \mathrm{S}^{1}\right\}
$$

and $\mathrm{S}_{l}^{1} \cap \mathrm{Sp}(1)$ is trivial. It is clear from this that $\mathrm{K}_{+} \simeq \mathrm{S}^{1} \mathrm{Sp}(1) \mathrm{Sp}(n-2)$, a contradiction to the original assumption $\mathrm{K}_{+} \simeq \mathrm{U}(2) \operatorname{Sp}(n-2)$ (note 
though that the case $\mathrm{K}_{+} \simeq \mathrm{S}^{1} \mathrm{Sp}(1) \mathrm{Sp}(n-2)$ will be treated later, and not give a primitive example).

3b) We choose $\mathrm{K}_{+}$such that $\mathrm{SU}(n-1) \subset \mathrm{K}_{+}$is the lower right block. If we assume $\mathrm{SU}(n-1) \subset \mathrm{H}_{+}$, then by primitivity we have $\mathrm{K}_{-} \simeq \mathrm{U}(n)$, which is treated in case $2 \mathrm{a}$. So we can assume $\mathrm{SU}(n-1) \not \subset \mathrm{H}_{+}$, which implies $\mathrm{H}=\mathrm{T}^{2} \mathrm{SU}(n-2)$. If $\mathrm{SU}(n-2) \not \subset \mathrm{H}_{-}$, then $\mathrm{K}_{-} \simeq \mathrm{S}^{1} \mathrm{U}(n-1)$. By primitivity, $\mathrm{SU}(n-1) \subset \mathrm{K}_{-}$is the upper left block. But $\mathrm{K}_{ \pm} \subset \mathrm{U}(n)$ where $\mathrm{U}(n)$ is either standard or has center $\operatorname{diag}(\bar{z}, z, \ldots, z)$.

So from now on we can assume $\mathrm{SU}(n-2) \subset \mathrm{H}_{-}$, i.e. $\mathrm{H} / \mathrm{H}_{-} \simeq \mathrm{S}^{1}$. This implies $l_{-}=2$ or $l_{-}=3$, and we divide cases by $\mathrm{K}_{-}$:

- If $l_{-}=2$ and $\mathrm{K}_{-}=\mathrm{SU}(2) \mathrm{U}(n-2)$, we reconjugate the diagram to make $\mathrm{K}_{-}$standard. Then $\mathrm{H}=\mathrm{S}_{1}^{1} \mathrm{U}(n-2)$ where $\mathrm{U}(n-2)$ is the lower right block and

$$
\mathrm{S}_{1}^{1}=\left\{\operatorname{diag}(z, \bar{z}, 1, \ldots, 1) \mid z \in \mathrm{S}^{1}\right\}
$$

This way we see there are 2 choices for $\mathrm{SU}(n-1) \subset \mathrm{K}_{+}$, both of which are equivalent. Now we claim that the factor $\mathrm{SU}(2) \subset \mathrm{K}_{-}$ is uniquely determined by $\mathrm{S}_{1}^{1}$ up to conjugation in $N\left(\mathrm{~S}_{1}^{1}\right)_{0}$, which finishes this case, because then $\mathrm{K}_{ \pm} \subset \mathrm{U}(n)$ up to conjugation in $N(\mathrm{H})_{0}$.

To prove the claim we restrict the discussion to the upper left $\operatorname{Sp}(2)$ block. We have $\mathrm{Sp}(2) \simeq \operatorname{Spin}(5)$, but $\mathrm{SU}(2) \subset \mathrm{Sp}(2)$ contains the central element, so it corresponds to $\mathrm{SO}(3) \subset \mathrm{SO}(5)$, and $\mathrm{S}^{1} \subset$ $\mathrm{SU}(2) \subset \mathrm{Sp}(2)$ to $\mathrm{SO}(2) \subset \mathrm{SO}(3) \subset \mathrm{SO}(5)$. But $\mathrm{SO}(3) \subset \mathrm{SO}(5)$ is uniquely determined up to conjugation in $N(\mathrm{SO}(2))$. So there is no primitive example in this case.

- If $l_{-}=2$ and $\mathrm{K}_{-}=\operatorname{Sp}(1) \mathrm{SU}(n-2) \mathrm{S}^{1}$, we claim $\mathrm{K}_{ \pm} \subset \operatorname{Sp}(1) \operatorname{Sp}(n-$ 1). As above, we restrict the discussion to the upper left $\operatorname{Sp}(2)$ block to show that $\mathrm{Sp}(1)$ is uniquely determined by $\mathrm{S}^{1}$ it contains. In $\mathrm{SO}(5), \mathrm{SO}(2)$ has either three 1-dimensional trivial subrepresentations, and the $\mathrm{SU}(2)$ containing it is unique in $N(\mathrm{SO}(2))_{0}$, or only one, but then the $\mathrm{SU}(2)$ containing it is already determined. This shows the claim if $S p(1)$ does not contain the central element of $\operatorname{Sp}(2)$. If $\mathrm{Sp}(1)$ contains the central element of $\mathrm{Sp}(2)$, the discussion above shows that is is also uniquely determined by the $S^{1}$ it contains. Again, no primitive example arises in this case. 
- If $l_{-}=3$ then either $\mathrm{K}_{-}=\mathrm{U}(2) \mathrm{U}(n-2)$ or $\mathrm{K}_{-}=\mathrm{S}^{1} \mathrm{Sp}(1) \mathrm{U}(n-2)$ we can use the same arguments as above to show that the manifold is not primitive.

3c) Suppose $\mathrm{S}^{1} \mathrm{Sp}\left(n_{1}\right) \subset \mathrm{H}_{+}$, so that $\mathrm{H}=\mathrm{S}^{1} \mathrm{Sp}\left(n_{1}\right) \mathrm{Sp}\left(n_{2}-1\right)$. We have $\mathrm{S}^{1} \mathrm{Sp}\left(n_{1}\right) \not \subset \mathrm{H}_{-}$by primitivity, so that $\mathrm{K}_{+} \simeq \mathrm{S}^{1} \mathrm{Sp}\left(n_{1}+1\right) \mathrm{Sp}\left(n_{2}-1\right)$. But the $\mathrm{S}^{1}$ factor of $\mathrm{H}$ is not diagonally embedded into the factors $\mathrm{S}^{1} \mathrm{Sp}\left(n_{1}+1\right)$ so that $\mathrm{S}^{1} \subset \mathrm{K}_{-}$can only act trivially on $\mathrm{S}^{l_{-}}$, which implies $\mathrm{S}^{1} \subset \mathrm{H}_{-}$, a contradiction.

$\operatorname{Sp}\left(n_{1}\right) \operatorname{Sp}\left(n_{2}\right)$ can only occur as the isotropy group of a transitive effective action on a sphere in the case $n_{1}=1$. But even in that case $\mathrm{H}=\mathbb{Z}_{k} \mathrm{Sp}\left(n_{1}\right) \mathrm{Sp}\left(n_{2}\right)$ cannot result in $\mathrm{K}_{-}=\operatorname{Sp}(1) \operatorname{Sp}\left(n_{2}+1\right)$ because as above $\operatorname{Sp}(1) \subset \mathrm{H}$ is not diagonally embedded into $\mathrm{K}_{-}$.

So now we can assume $\mathrm{H}=\operatorname{Sp}\left(n_{1}-1\right) \operatorname{Sp}\left(n_{2}\right) \Delta \mathrm{S}_{l}^{1}$, where we assume $\operatorname{Sp}\left(n_{1}\right)$ in the upper left block, $\operatorname{Sp}\left(n_{2}\right)$ in the lower right, and

$$
\Delta \mathrm{S}_{l}^{1}=\left\{\operatorname{diag}(\underbrace{1, \ldots, 1}_{n_{1}-1}, z^{l}, z, \underbrace{1, \ldots, 1}_{n_{2}}) \mid z \in \mathrm{S}^{1}\right\}
$$

Note that this choice actually means $\mathrm{K}_{+}=\operatorname{Sp}\left(n_{1}\right) \mathrm{S}^{1} \mathrm{Sp}\left(n_{2}\right)$ so that $\mathrm{S}^{1}$ acts on the $\left(n_{1}+1\right)$ st coordinate. If $|l| \neq 1$, this implies $\mathrm{K}_{-}=$ $\operatorname{Sp}\left(n_{1}-1\right) \mathcal{S}^{1} \sigma\left(\operatorname{Sp}\left(n_{2}+1\right)\right)$, where $\sigma$ exchanges the Koordinates $n_{1}$ and $n_{1}+1$. This is not primitive, for $\mathrm{K}_{ \pm} \subset \varphi\left(\mathrm{S}^{1} \mathrm{Sp}(n-1)\right)$ where $\varphi$ exchanges the first with the $\left(n_{1}+1\right)$ st coordinate. If $|l|=1$, we can actually have $\mathrm{Sp}\left(n_{2}+1\right) \subset \mathrm{K}_{-}$embedded as the lower right block, but in this case it's even easier to see that the resulting manifold is not primitive.

3d) This case is completely analogous to $3 \mathrm{c}$ (for $l=1$ ) in showing that the only possibility is $\mathrm{H}=\mathrm{Sp}\left(n_{1}-1\right) \mathrm{Sp}\left(n_{2}\right)$ which does not lead to a primitive manifold again (because $\mathrm{K}_{ \pm} \subset \mathrm{Sp}(1) \mathrm{Sp}(n-1)$ ).

3e) First suppose $\mathrm{Sp}(n-2) \subset \mathrm{H}_{+}$. This implies $\mathrm{Sp}(n-1) \subset \mathrm{K}_{-}$and $\mathrm{K}_{-}$ is a subgroup of maximal rank, so we can refer to one of the previous cases.

So we can assume $\mathrm{H}=\mathrm{T}^{2} \mathrm{Sp}(n-3)$. If $\mathrm{Sp}(n-3) \not \subset \mathrm{H}_{-}$, we have $\mathrm{K}_{-} \simeq \mathrm{K}_{+}$, and we claim that this is not a primitive manifold. For this note that both $\mathrm{K}_{ \pm}$(acting on $\mathbb{H}^{n}$ ) have 2 one-dimensional subrepresentations, while $\mathrm{H}$ has 3 , which contain the ones of $\mathrm{K}_{ \pm}$. So we see that $\mathrm{K}_{ \pm}$have a common one-dimensional subrepresentation, which shows $\mathrm{K}_{ \pm} \subset \mathrm{Sp}(1) \operatorname{Sp}(n-1)$, so the manifold is not primitive. 
We are left with the case $\operatorname{Sp}(n-3) \subset \mathrm{H}_{-}$, which implies $l_{-}=2$ or $l_{-}=3$. We divide cases by $\mathrm{K}_{+}$

- If $l_{-}=2$ and $\mathrm{K}_{-} \simeq \mathrm{SO}(3) \mathrm{S}^{1} \mathrm{Sp}(n-3)$, we have $\mathrm{S}^{1} \subset N(\mathrm{SO}(3) \operatorname{Sp}(n-$ $3)$. We reconjugate the diagram so that $\mathrm{K}_{-}$is standard and $\mathrm{SO}(2) \subset \mathrm{H} / \mathrm{H}_{-}$is the upper left block. Then furthermore conjugate the diagram by the matrix having

$$
\left(\begin{array}{cc}
1 & i \\
1 & -i
\end{array}\right)
$$

in the upper left block, extended by the identity matrix $E_{n-2}$. This conjugates $\mathrm{SO}(2) \subset \mathrm{H}$ into

$$
\left\{\operatorname{diag}(z, \bar{z}, 1, \ldots, 1) \mid z \in \mathrm{S}^{1}\right\}
$$

Now $\mathrm{S}^{1} \subset \mathrm{H}_{-}$is in $N(\mathrm{SO}(3) \mathrm{Sp}(n-3))$ and therefore $\mathrm{T}^{2} \subset \mathrm{H}$ is given by

$$
\left\{\operatorname{diag}(z, z, z, 1, \ldots, 1) \mid z \in \mathrm{S}^{1}\right\} \cdot\left\{\operatorname{diag}(z, \bar{z}, 1, \ldots, 1\} \mid z \in \mathrm{S}^{1}\right\}
$$

This completely determines $\mathrm{K}_{+}$and we find one primitive example.

- If $l_{-}=2$ and $\mathrm{K}_{-} \simeq \mathrm{SU}(2) \mathrm{S}^{1} \mathrm{Sp}(n-3)$, we reconjugate the diagram to put $\mathrm{K}_{-}$into standard form, which gives $\mathrm{H}=\mathrm{S}_{1}^{1} \mathrm{~S}_{2}^{1} \mathrm{Sp}(n-3)$ where $\operatorname{Sp}(n-3)$ is the lower right block and

$$
\begin{aligned}
& \mathrm{S}_{1}^{1}=\left\{\operatorname{diag}(z, \bar{z}, 1, \ldots, 1) \mid z \in \mathrm{S}^{1}\right\} \\
& \mathrm{S}_{2}^{1}=\left\{\operatorname{diag}(1,1, z, 1, \ldots, 1) \mid z \in \mathrm{S}^{1}\right\}
\end{aligned}
$$

From this it is clear that $\mathrm{Sp}(n-2) \subset \mathrm{K}_{+}$is the lower right block. But $\mathrm{H} \cap \mathrm{Sp}(n-2) \neq \mathrm{Sp}(n-3)$, so $\mathrm{K}_{+} / \mathrm{H}$ is not a sphere.

- If $l_{-}=3$ and $\mathrm{K}_{-} \simeq \mathrm{U}(2) \mathrm{S}^{1} \mathrm{Sp}(n-3)$, we again reconjugate the diagram to make $\mathrm{K}_{-}$standard. Then $N(\mathrm{H})_{0}$ contains $\mathrm{SO}(3)$, so we can assume that $\mathrm{Sp}(n-2) \subset \mathrm{K}_{+}$is the lower right block, so we have $\mathrm{K}_{ \pm} \subset \mathrm{Sp}(2) \mathrm{Sp}(n-2)$, and the manifold is not primitive.

4a) The fact that $\mathrm{U}(n)$ is given as the centralizer of its center $\mathrm{S}^{1}$ in $\operatorname{Sp}(n)$ leads to the same reasoning as in case 4 a of 9 on page 58 to show that no primitive manifolds arise in this case. 
4b-4d) We will show that no primitive manifold can arise in these cases, because both $\mathrm{K}_{ \pm}$have a common one-dimensional subrepresentation when acting on $\mathbb{H}^{n}$, showing $\mathrm{K}_{ \pm} \subset \mathrm{Sp}(1) \mathrm{Sp}(n-1)$. For this, denote the number of one-dimensional subrepresentations of the standard representation of a subgroup $\mathrm{H} \hookrightarrow \mathrm{Sp}(n)$ by $s(\mathrm{H})$. Note that if $\mathrm{K}_{ \pm}$acts on a one-dimensional subspace of $\mathbb{H}^{n}$, so does $\mathrm{H}$.

- If $n_{1}, n_{2} \geq 2$, then $s\left(\mathrm{~K}_{+}\right)=2, s(\mathrm{H})=3, s\left(\mathrm{~K}_{-}\right)=2$ and it is clear that both $\mathrm{K}_{ \pm}$share a one-dimensional invariant subspace.

- If $n_{1}=1$ and $\mathrm{Sp}\left(n_{1}\right) \not \subset \mathrm{H}_{+}$, then $s\left(\mathrm{~K}_{+}\right)=3, s(\mathrm{H})=3, s\left(\mathrm{~K}_{-}\right)=2$ and it is clear that the claim holds.

- If $n_{1}=1, \operatorname{Sp}\left(n_{1}\right) \subset \mathrm{H}_{+}$and $n_{2}>1$, then $s\left(\mathrm{~K}_{+}\right)=3, s(\mathrm{H})=$ $4, s\left(\mathrm{~K}_{-}\right)=3$ and the claim holds.

- If $n_{1}=1=n_{2}$, then $s\left(\mathrm{~K}_{+}\right)=4=s(\mathrm{H}), s\left(\mathrm{~K}_{-}\right)=3$ and the claim holds.

4e) Suppose $\mathrm{H}=\Delta \mathrm{Sp}(1) \operatorname{Sp}\left(n_{1}-1\right) \mathrm{U}\left(n_{2}\right)$. Then $\mathrm{SU}\left(n_{2}+1\right) \subset \mathrm{K}_{-} \cap$ $N\left(\Delta \operatorname{Sp}(1) \operatorname{Sp}\left(n_{1}-1\right)\right.$, a contradiction. So we can assume $\mathbf{H}=\operatorname{Sp}(1) \operatorname{Sp}\left(n_{1}\right) \mathrm{U}\left(n_{2}-\right.$ $1)_{k}$ for some $k \in \mathbb{Z}$. Then $\mathrm{K}_{-}=\operatorname{Sp}(1) \operatorname{Sp}\left(n_{1}+1\right) \mathrm{U}\left(n_{2}-1\right)$ and $k=1$. If $n_{2} \geq 2$, this is exactly the previous case by exchanging $\mathrm{K}_{-}$and $\mathrm{K}_{+}$. If $n_{2}=2$, then $N(\mathrm{H}) \supset \mathrm{SO}(3)$, so we can assume $\mathrm{Sp}\left(n_{1}+1\right) \subset \mathrm{K}_{-}$is the upper left block, which shows $\mathrm{K}_{ \pm} \subset \mathrm{Sp}\left(n_{1}+1\right) \mathrm{Sp}\left(n_{2}\right)$.

4f) First assume $\mathrm{H}=\mathrm{T}^{2} \mathrm{Sp}\left(n_{1}-1\right) \mathrm{SU}\left(n_{2}\right)$. This implies $\mathrm{K}_{-} \simeq \mathrm{T}^{2} \mathrm{Sp}\left(n_{1}-\right.$ 1) $\mathrm{SU}\left(n_{2}+1\right)$ by the classification of actions on a sphere (note that this is true even in the case $n_{2}=2$, because $\mathrm{Sp}(2) / \mathrm{SU}(2)$ is not a sphere). By primitivity, $\mathrm{SU}\left(n_{2}+1\right) \subset \mathrm{K}_{-}$is completely determined for $n_{1}>1$ and we have $\mathrm{K}_{ \pm} \subset \mathrm{Sp}\left(n_{1}\right) \mathrm{Sp}\left(n_{2}+1\right)$, so the manifold is not primitive. If $n_{1}=1$, we have $\mathrm{SO}(2) \subset N(\mathrm{H})_{0}$, so we can assume that $\mathrm{SU}\left(n_{2}+1\right) \subset \mathrm{K}_{-}$is the lower right block and the claim holds, too.

The second possibility is $\mathrm{H}=\mathrm{T}^{2} \mathrm{Sp}\left(n_{1}\right) \mathrm{SU}\left(n_{2}-1\right)$. If $\mathrm{K}_{-} \simeq \mathrm{T}^{2} \mathrm{Sp}\left(n_{1}+\right.$ 1)SU $\left(n_{2}+1\right)$ and $n_{2}>2$ we can argue as before to show $\mathrm{K}_{ \pm} \subset \mathrm{Sp}\left(n_{1}+\right.$ 1) $\mathrm{Sp}\left(n_{2}\right)$. If in addition $n_{2}=2$, we have $\mathrm{SO}(3) \subset N(\mathrm{H})_{0}$ so we can assume $\mathrm{Sp}(n-2) \subset \mathrm{K}_{-}$is the upper left block, and the claim holds. 


\section{$9 \mathrm{G}=\mathrm{SO}(2 n), n \geq 4$}

For a general description of the classification procedure, see section 3 on page 20 .

We claim that for $n \geq 4$ the simply connected primitive cohomogeneity one $\mathrm{SO}(2 n)$-manifolds with positive euler characteristic are given by table 12

Table 12: $\mathrm{SO}(2 n)$-cohomogeneity one manifolds for $n \geq 4$

\begin{tabular}{|c|}
\hline $\mathrm{S}^{1} \mathrm{SU}(n-1) \subset \sigma(\mathrm{U}(n)), \mathrm{U}(n)$ \\
where $\mathrm{S}^{1}=\{\operatorname{diag}(z, 1, \ldots, 1)\}$ or $\mathrm{S}^{1}=\{\operatorname{diag}(1, z, \ldots, z)\}$ and \\
$\sigma$ is conjugation by $\operatorname{diag}(-1,1, \ldots, 1)$
\end{tabular}

The possibilities for $\mathrm{K}_{+}$are given by table 13 .

If a group of complex matrices is involved (e.g. $\mathrm{U}(n)$ ), we will deliberately use complex notation for the corresponding real matrices. In particular, for $e^{i \varphi}=z \in S^{1}$, we will use $\operatorname{diag}(z, \ldots, z)$ for the matrix containing $\left(\begin{array}{cc}\cos \varphi & \sin \varphi \\ -\sin \varphi & \cos \varphi\end{array}\right)$ on the diagonal $2 \times 2$-blocks and 0 everywhere else.

2a) If $\mathrm{SU}(n) \subset \mathrm{H}_{+}$, we have $\mathrm{SU}(n+1) \subset \mathrm{K}_{-}$, a contradiction to $\mathrm{K}_{-}$being a subgroup of $\mathrm{SO}(2 n)$. So $\mathrm{H}$ is given by $\mathrm{S}_{k}^{1} \mathrm{SU}(n-1)$, where $\mathrm{S}_{k}^{1}=$ $\left\{\operatorname{diag}\left(z^{(k+1)(n-1)}, z^{-k}, \ldots, z^{-k}\right)\right\}$ by subsection B.2 on page 66 of the appendix. We have $N(\mathrm{SU}(n-1))_{0}=\mathrm{S}^{1} \mathrm{U}(n-1) \subset \mathrm{K}_{+}$, therefore $\mathrm{SU}(n-1) \not \subset \mathrm{H}_{-}$and therefore $\mathrm{K}_{-}$is isomorphic to $\mathrm{U}(n)$. 
Table 13: Possibilities for $K_{+}$

\begin{tabular}{|c|l|l|}
\hline Factors & Group & Conditions \\
\hline 2 & $\mathrm{U}(n)$ & - \\
\hline 2 & $\mathrm{SO}\left(2 n_{1}\right) \mathrm{SO}\left(2 n_{2}\right)$ & $n_{1}, n_{2}>2, n_{1}+n_{2}=n$ \\
\hline 2 & $\mathrm{SO}(2) \mathrm{SO}(2 n-2)$ & - \\
\hline 3 & $\mathrm{U}\left(n_{1}\right) \mathrm{SO}\left(2 n_{2}\right)$ & $n_{1}>1, n_{2}>2, n_{1}+n_{2}=n$ \\
\hline 3 & $\mathrm{U}(n-1) \mathrm{SO}(2)$ & - \\
\hline 3 & $\mathrm{SO}(2 n-4) \mathrm{SO}(4)$ & $n>4$ \\
\hline 4 & $\mathrm{U}\left(n_{1}\right) \mathrm{U}\left(n_{2}\right)$ & $n_{1}, n_{2}>1, n_{1}+n_{2}=n$ \\
\hline 4 & $\mathrm{U}(n-2) \mathrm{SO}(4)$ & - \\
\hline 4 & $\mathrm{SO}(4) \mathrm{SO}(4)$ & $n=4$ \\
\hline
\end{tabular}

The center of $\mathrm{K}_{-}$can be conjugated into the standard diagonal $S^{1}$, and must of course commute with $\mathrm{SU}(n-1)$, so it is determined up to the first complex coordinate, which can be $z$ or $z^{-1}$. By primitivity, $\mathrm{K}_{-} \neq \mathrm{K}_{+}$, so the center of $\mathrm{K}_{-}$is given by $z \mapsto \operatorname{diag}\left(z^{-1}, z, \ldots, z\right)$, and therefore $\mathrm{K}_{-}=\varphi(\mathrm{U}(n))$, where $\varphi$ is conjugation by $\operatorname{diag}(-1,1,1, \ldots, 1)$. Since $\varphi$ only fixes $\mathrm{H}$ for $k=0,-1$, we are left with 2 comomogeneity1-manifolds, both of which are primitive indeed: Conjugation by

$$
\operatorname{diag}(-1,1, \ldots, 1)
$$

on $\mathrm{U}(n)$ has the same image as conjugation by

$$
\operatorname{diag}(1,1,-1,1, \ldots,-1,1)
$$

(they only differ by $\operatorname{diag}(-1,1, \ldots,-1,1)$, which is complex conjugation), but this restricts to complex conjugation on $\mathrm{U}(n-1)$, which is an outer automorphism and cannot possibly come from an element of $N(\mathrm{H})_{0}=\mathrm{S}^{1} \mathrm{U}\left(n_{1}\right)$. Note that both of $\mathrm{K}_{ \pm}$must be connected, for $l_{ \pm}=2 n-1>1$.

2b) If $\mathrm{K}_{+}=\mathrm{SO}\left(2 n_{1}\right) \mathrm{SO}\left(2 n_{2}\right)$ with $n_{1}, n_{2}>2$, we may assume that $\mathrm{H}=$ $\mathrm{SO}\left(2 n_{1}\right) \mathrm{SO}\left(2 n_{2}-1\right)$ is standard, and since $l_{+}=2 n_{1}-1>1$ we know $\mathrm{K}_{-}$is connected. Since the action of $\mathbf{S O}(2 n)$ is primitive and 
almost effective, we have $\mathrm{K}_{-}=\mathrm{SO}\left(2 n_{1}+1\right) \mathrm{SO}\left(2 n_{2}-1\right)$. This is the primitive action of $\mathrm{SO}(2 n)$ on the Grassmanian $\mathrm{G}_{2 n_{2}}\left(\mathbb{R}^{2 n+1}\right)=$ $\mathrm{SO}(2 n+1) / \mathrm{SO}\left(2 n_{1}+1\right) \mathrm{SO}\left(2 n_{2}\right)$

2c) $\mathrm{K}_{+} / \mathrm{H}_{+}$is not as $\mathrm{O}(2 n-2)$ since $\mathrm{O}(2 n-2)$ is not the direct product of $\mathrm{SO}(2 n-2)$ and $\mathbb{Z}_{2}$. If $\mathrm{K}_{+} / \mathrm{H}_{-}$is $\mathrm{SO}(2 n-2)$, this is the exact same as case $2 \mathrm{~b})$, giving $\mathrm{K}_{-}=\mathrm{SO}(3) \mathrm{SO}(2 n-3)$. So $\mathrm{K}_{+} / \mathrm{H}_{-}=\mathrm{S}^{1}$, which implies $\mathrm{H}=\mathbb{Z}_{k} \mathrm{SO}(2 n-2)$ for some $k$. Now $\mathrm{H}_{-}$can't contain $\mathrm{SO}(2 n-2)$, so $K_{-}$must contain $\mathrm{SO}(2 n-1)$ as a transitively acting factor. Since the normalizer of $\mathrm{SO}(2 n-1)$ in $\mathrm{SO}(2 n)$ is $\mathrm{S}(\mathrm{O}(1) \mathrm{O}(2 n-1))$ we are restricted to $k=1$ or $k=2$ (note that $l_{+}=1$, so actually components may occur). For $k=1$ this is analogous to case $2 \mathrm{~b}$ ), the action of $\mathrm{SO}(2 n)$ on the Grassmanian $\mathrm{SO}(2 n+1) / \mathrm{SO}(2 n-1) \mathrm{SO}(2)$. For $k=2$ we know $\mathrm{SO}(2 n-1) \mathbb{Z}_{2}$ is isomorphic to $\mathrm{O}(2 n-1)$ (since $\mathbb{Z}_{2}$ acts by an inner automorphism), but $\mathrm{K}_{-} / \mathrm{H}_{-}$is not $\mathrm{O}(2 n-1)$ since this would imply $\mathrm{H}_{/} \mathrm{H}_{-} \simeq \mathrm{O}(2 n-2)$ which is not isomorphic to $\mathrm{SO}(2 n-2) \times \mathbb{Z}_{2}$, which in turn $\mathrm{H}$ is. So $\mathrm{K}_{-} / \mathrm{H}_{-}=\mathrm{SO}(2 n-1)$, giving a non-effective action.

3a) $\mathrm{K}_{+} / \mathrm{H}_{+}$is not $\mathrm{SO}\left(2 n_{2}\right)$, since that would imply that $\mathrm{K}_{-}$has $\mathrm{SU}\left(n_{1}+1\right)$ as a simple factor as well as $\mathrm{SO}\left(2 n_{2}-1\right)$. So $\mathrm{K}_{+} / \mathrm{H}_{+}=\mathrm{U}\left(n_{1}\right)$, and therefore $\mathrm{H}=\mathrm{U}\left(n_{1}-1\right)_{k} \mathrm{SO}\left(2 n_{2}\right)$. Since the action is primitive and almost effective, $\mathrm{K}_{-} / \mathrm{H}_{-}$must contain $\mathrm{SO}\left(2 n_{2}+1\right)$ as a simple factor, and since $l_{+}=2 n_{1}+1>1, \mathrm{~K}_{-}$must be connected. So $\mathrm{K}_{-}=\mathrm{U}\left(n_{1}-1\right)_{k} \mathrm{SO}\left(2 n_{2}+\right.$ 1 ), but the normalizer of $\mathrm{U}\left(n_{1}-1\right)_{k}$ does not contain $\mathrm{SO}\left(2 n_{2}+1\right)$ save the case $k=0$. The embedding of $\mathrm{K}_{-}$and $\mathrm{H}$ into $\mathrm{SO}(2 n)$ gives a faithfull representation, and we can see that there is a 2-dimensional trivial subrepresentation for $\mathrm{H}$, while there is a 1-dimensional trivial subrepresentation for $\mathrm{K}_{-}$. It is clear that the latter is contained in the former and determines $\mathrm{K}_{-}$. But $N(\mathrm{H})_{0}=\mathrm{U}\left(n_{1}-1\right) \mathrm{SO}(2) \mathrm{SO}\left(2 n_{2}\right)$ acts transitively on the 2-dimensional trivial representation (via the factor $\mathrm{SO}(2)$ ), so by conjugating with an element of $N(\mathrm{H})_{0}$ we can achieve $\mathrm{K}_{-}$ is standard.

This is the action of $\mathrm{SO}(2 n)$ on $\mathrm{SO}(2 n+1) / \mathrm{U}\left(n_{1}\right) \mathrm{SO}\left(2 n_{2}+1\right)$, which is primitive because $\mathrm{K}_{+}$is a maximal connected subgroup of $\mathrm{SO}\left(2 n_{1}\right) \mathrm{SO}\left(2 n_{2}\right)$ which in turn is maximal connected in $\mathrm{SO}(2 n)$. Both of these do not contain $\mathrm{K}_{-}$, even up to components.

3b) In addition to the possibility from 3a) (giving the action of $\mathrm{SO}(2 n)$ on $\mathrm{SO}(2 n+1) / \mathrm{U}(n-1) \mathrm{SO}(3))$, there are 2 other possibilities. First, if $\mathrm{H}=\mathrm{U}(n-2) \mathrm{SO}(2)$, we can have $\mathrm{K}_{-}=\mathrm{U}(n-2) \mathrm{U}(2)$. From the 
isotropy representation of $\mathrm{H}$ in $\mathrm{G}$ we see that $\mathrm{U}(2)$ is contained in the lower right $\mathrm{SO}(4)$ block. It is determined by $\mathrm{SU}(2) \subset \mathrm{U}(2)$, and it is known that $\mathrm{SO}(4)=\mathrm{SU}(2) \mathrm{SU}(2)$, so there are 2 choices for the $\mathrm{SU}(2)$ factor. One of those leads to $\mathrm{K}_{-}$being contained in $\mathrm{U}(n)$, not giving a primitive manifold (for $\mathrm{K}_{+} \subset \mathrm{U}(n)$ as well). The other one is obtained from the first by an outer automorphism, given by conjugation with $\operatorname{diag}(1, \ldots, 1,-1)$. But this automorphism fixes both $\mathrm{H}$ and $\mathrm{K}_{+}$, so the resulting manifold is equivalent to the first one.

Secondly we can have $\mathrm{K}_{+} / \mathrm{H} \simeq \mathrm{U}(n-1)$, so that $\mathrm{H}=\mathrm{T}^{2} \mathrm{SU}(n-2)$. First assume $\mathrm{SU}(n-2) \not \subset \mathrm{H}_{-}$, which implies $\mathrm{K}_{+} \simeq \mathrm{K}_{-}$. Both $\mathrm{K}_{ \pm}$have a 2-dimensional subrepresentation from their embedding into $\mathrm{SO}(2 n)$, which is neccessarily given by $\mathbb{R} e_{1} \oplus \mathbb{R} e_{2}$ or $\mathbb{R} e_{3} \oplus \mathbb{R} e_{4}$, and we chose $\mathrm{K}_{+}$the way that this is $\mathbb{R} e_{1} \oplus \mathbb{R} e_{2}$. If it's the same for $\mathrm{K}_{-}$, we have $\mathrm{K}_{ \pm} \subset \mathrm{U}(n)$, so the manifold is not primitive. If it is $\mathbb{R} e_{3} \oplus \mathbb{R} e_{4}$, we look at the center of $\mathrm{U}(n-1) \subset \mathrm{K}_{-}$. If it is given by

$$
\left\{\operatorname{diag}(z, 1,1, z, \ldots, z) \mid z \in \mathrm{S}^{1}\right\}
$$

we again have $\mathrm{K}_{ \pm} \subset \mathrm{U}(n)$. If it is given by

$$
\left\{\operatorname{diag}(\bar{z}, 1,1, z, \ldots, z) \mid z \in \mathrm{S}^{1}\right\}
$$

we have $\mathrm{K}_{ \pm} \subset \sigma(\mathrm{U}(n))$, where $\sigma$ is conjugation by $\operatorname{diag}(-1,1, \ldots, 1)$. So now we can assume $\mathrm{SU}(n-2) \subset \mathrm{H}_{-}$, which implies $\mathrm{H} / \mathrm{H}_{-}=\mathrm{S}^{1}$, and therefore $l_{-}=2$ or $l_{-}=3$. We divide cases by $K_{-}$:

- If $\mathrm{K}_{-}=\mathrm{S}^{1} \mathrm{SU}(2) \mathrm{SU}(n-2)$, we look at the centralizer of $\mathrm{SU}(2)$ in the upper left $\mathrm{SO}(4)$-block: It is either given by $\operatorname{diag}(z, z)$, so that $\mathrm{K}_{ \pm} \subset \mathrm{U}(n)$, or $\operatorname{diag}(\bar{z}, z)$, which implies $\mathrm{K}_{ \pm} \subset \sigma(\mathrm{U}(n))$, where $\sigma$ is as above.

- If $\mathrm{K}_{-}=\mathrm{SO}(3) \mathrm{S}^{1} \mathrm{SU}(n-2)$ with $\mathrm{SO}(3) \subset N\left(\mathrm{~S}^{1} \mathrm{SU}(n-2)\right)$, we can reconjugate the diagram to achieve that $\mathrm{K}_{-}$acts trivially on $\mathbb{R} e_{4}$. This implies $\mathrm{H}=\mathrm{S}_{1}^{1} \mathrm{~S}_{2}^{1} \mathrm{SU}(n-2)$ with $\mathrm{SU}(n-2)$ in the lower right block and

$$
\begin{aligned}
& \mathrm{S}_{1}^{1}=\left\{\operatorname{diag}(z, 1, \ldots, 1) \mid z \in \mathrm{S}^{1}\right\} \\
& \mathrm{S}_{2}^{1}=\left\{\operatorname{diag}(1,1,1,1, z, \ldots, z) \mid z \in \mathrm{S}^{1}\right\}
\end{aligned}
$$

We note that $\mathrm{SU}(n-1) \subset \mathrm{K}_{+}$is in $N\left(\mathrm{~S}_{1}^{1}\right)$, so it is in the lower right block. This gives one primitive example. 
- If $\mathrm{K}_{-} \simeq \mathrm{U}(2) \mathrm{U}(n-2)$, we can use the same reasoning above to see that the manifold is not primitive.

$3 c)$ There are 2 actions arising in the same way as described in $2 b$ ), namely $\mathrm{SO}(2 n)$ acting on either $\mathrm{SO}(2 n+1) / \mathrm{SO}(2 n-3) \mathrm{SO}(4)$ or $\mathrm{SO}(2 n+$ $1) / \mathrm{SO}(2 n-4) \mathrm{SO}(5)$. We only need to note that $l_{+}$is odd by corollary 1.7 on page 15 .

4a) None of the actions arising are primitive. We have

$$
\mathrm{H}=\mathrm{T}^{2} \mathrm{SU}\left(n_{1}\right) \mathrm{SU}\left(n_{2}-1\right) \text { and } \mathrm{H}_{+}=\mathrm{S}^{1} \mathrm{SU}\left(n_{1}\right)
$$

Since $\mathrm{H}_{-} \cap \mathrm{H}_{+}$is finite, $\mathrm{SU}\left(n_{1}\right) \subset \mathrm{H}$ cannot act trivial in $\mathrm{K}_{-}$, and therefore $K_{-}$is isomorphic to $\mathrm{U}\left(n_{1}+1\right) \mathrm{U}\left(n_{2}-1\right)$. As in case $\left.2 \mathrm{a}\right)$ we can determine the embedding via the center. The standard embedding yields $\mathrm{K}_{-}, \mathrm{K}_{+} \subset \mathrm{U}(n)$ and therefore not a primitive action, so the center has to be given by

$$
\left(z_{1}, z_{2}\right) \mapsto(\underbrace{z_{1}, \ldots, z_{1}}_{n_{1}}, z_{1}^{-1}, z_{2}, \ldots, z_{2})
$$

which implies that bot $K_{-}$and $K_{+}$are contained in the subgroup of $\mathrm{SO}(2 n)$ isomorphic to $\mathrm{U}(n)$ with center

$$
\left\{(\underbrace{z, \ldots, z}_{n_{1}}, z^{-1}, z^{-1}, \ldots, z^{-1}) \mid z \in \mathrm{S}^{1}\right\}
$$

4b) This case is completely analogous to case 3a), because $\mathrm{SO}(4)$ cannot act as $\mathrm{SO}(3)$ for the same reason as in $3 \mathrm{c})$.

4c) Again, no new example other than the ones arising from case $2 \mathrm{~b}$ can occur because $l_{+}$is odd by corollary 1.7 on page 15 .

\section{1 $\operatorname{Spin}(2 n), n \geq 4$}

For a general description of the classification procedure, see section 3 on page 20. Changes of this procedure for the Spin-groups are described in section 3.1 on page 20 .

We claim that there are no simply connected primitive cohomogeneity one $\operatorname{Spin}(2 n)$-manifolds with positive euler characteristic for $n \geq 4$ such that the element -1 (which projects to the identity, but is not the identity itself) does not act trivially (i.e. it is not an action of $\mathrm{SO}(2 n)$ ). 
First, it is easily seen that in the case $n=4$, there can only be examples that are coming from an $\mathrm{SO}(8)$-example. Consider the maximal torus $\mathrm{T}^{4}$ of Spin(8), containing the maximal torus $\mathrm{T}^{3}$ of $\mathrm{H}$ (the corank of $\mathrm{H}$ is 1 by corollary 1.7 on page 15$)$. The group of involutions $\mathbb{Z}_{2}^{4}$ in $\mathrm{T}^{4}$ contains the center $\mathbb{Z}_{2}^{2}$ of Spin(8), which therefore has nonempty intersection with the group of involutions $\mathbb{Z}_{2}^{3}$ of $\mathrm{T}^{3}$. Therefore, $\mathrm{H}$ contains an element of the center of Spin(8). Since the quotient of $\operatorname{Spin}(8)$ by any central $\mathbb{Z}_{2}$ is $\mathrm{SO}(8)$, this shows the claim.

Now we have reduced the table of possibilities for $\mathrm{K}_{+}$to those given in table 14 (where $\hat{\mathrm{U}}(n)$ is the preimage of $\mathrm{U}(n) \subset \mathrm{SO}(2 n)$ ), where $n>4$.

Table 14: Possibilities for $K_{+}$

\begin{tabular}{|c|l|l|}
\hline Factors & Group & Conditions \\
\hline 2 & $\hat{\mathbf{U}}(n)$ & - \\
\hline 3 & $\hat{\mathrm{U}}(n-1) \operatorname{Spin}(2)$ & - \\
\hline 3 & $\hat{\mathrm{U}}(n-3) \operatorname{Spin}(6)$ & - \\
\hline 4 & $\hat{\mathrm{U}}\left(n_{1}\right) \hat{\mathrm{U}}\left(n_{2}\right)$ & $n_{1}, n_{2}>1, n_{1}+n_{2}=n$ \\
\hline
\end{tabular}

The last case is easily dismissed as well, since we already know from section 9 on page 58 that no such manifold will be primitive (the proof carries over almost verbatim).

2a) We look at the projection of the diagram in $\mathrm{SO}(2 n)$. For the same reasons as in $2 \mathrm{a}$ ) of section 9 on page 58 we know $\mathrm{SU}(n) \not \subset \mathrm{H}$, so $\mathrm{H}=\mathrm{S}_{k}^{1} \mathrm{SU}(n-1)$, where

$$
\mathrm{S}_{k}^{1}=\left\{\operatorname{diag}\left(z^{(k+2)(n-1)}, z^{-k}, \ldots, z^{-k}\right) \mid z \in \mathrm{S}^{1}\right\}
$$

and $k$ is odd. As already shown in 2a) of section 9 on page 58 we have $K_{-} \simeq \mathrm{U}(n)$ as well, and since the diagram is primitive, $K_{-}=\varphi(\mathrm{U}(n))$ where $\varphi$ is the conjugation by $\operatorname{diag}(-1,1, \ldots, 1)$, which restricts to complex conjugation of the first component on $S_{k}^{1}$. The latter is only invariant under $\varphi$ in the cases $k=0,-2$, but the quotients are spheres in these cases and so there are no new cases here.

3a) As before, we will consider the situation in $\mathrm{SO}(2 n)$. As in case $3 \mathrm{a}$ ) of section 9 on page 58 , we have that $\mathrm{H}$ is given as $\mathrm{U}(n-2)_{k} \mathrm{SO}(2)$, where $\mathrm{U}(n-2)_{k}$ is determined by 


$$
\mathrm{S}_{k}^{1}:=\left\{\operatorname{diag}\left(1, z^{(k+2)(n-2)}, z^{-k}, \ldots, z^{-k}\right) \mid z \in \mathrm{S}^{1}\right\}
$$

with $k$ odd. Analogous to case $3 \mathrm{~b}$ ) of 9 on page 58 we know that $\mathfrak{k}_{-} / \mathfrak{h}_{-}$ is given by either $\mathfrak{s o}(3)$ or $\mathbb{R} \oplus \mathfrak{s u}(2)$. But both cases imply $k=-2$, a contradiction.

3b) Again, we will consider the diagram of subgroups of $\mathrm{SO}(2 n)$. If $\mathrm{K}_{+} / \mathrm{H}_{+}=$ $\mathrm{SU}(4)$, we have $\mathrm{H}=\mathrm{SU}(n-3) \mathrm{S}^{1} \mathrm{SU}(3)$, a contradiction, because $\mathrm{K}_{-}$ would have to contain $\mathrm{SU}(n-2)$, but there is no embedding of $\mathrm{SU}(n-$ $2) \mathrm{SU}(3)$ into $\mathrm{SO}(2 n)$. The same reasoning shows $\mathrm{H} \neq \mathrm{U}(n-3) \mathrm{O}(5)$. We are left with $\mathrm{H}=\mathrm{S}_{k}^{1} \mathrm{SU}(n-4) \mathrm{SO}(6)$ where $\mathrm{S}_{k}^{1}$ is given by

$$
\mathrm{S}_{k}^{1}=\left\{\operatorname{diag}(z^{(k+2)(n-4)}, z^{-k}, \ldots, z^{-k} \underbrace{1, \ldots, 1}_{6 \text { times }})\right\}
$$

with $k$ odd. But in that case $\mathrm{SO}(2 n) / \mathrm{H}$ is simply connected, a contradiction. 


\section{A Tables}

\begin{tabular}{|l|l|}
\hline $\mathrm{G}$ & $\mathrm{K}_{+}$ \\
\hline $\mathrm{SU}(n)$ & $\begin{array}{l}\mathrm{S}\left(\mathrm{U}\left(n_{1}\right) \mathrm{U}\left(n_{2}\right) \cdots \mathrm{U}\left(n_{k}\right)\right) \\
\text { where } n_{1}+n_{2}+\ldots+n_{k}=n\end{array}$ \\
\hline $\mathrm{SO}(2 n)$ & $\begin{array}{c}\mathrm{SO}\left(2 n_{1}\right) \cdots \mathrm{SO}\left(2 n_{k}\right) \mathrm{U}\left(m_{1}\right) \cdots \mathrm{U}\left(m_{l}\right) \\
\text { where } \sum n_{i}+\sum m_{i}=n\end{array}$ \\
\hline $\mathrm{SO}(2 n+1)$ & $\begin{array}{l}\mathrm{SO}(2 k+1) \mathrm{SO}\left(2 n_{1}\right) \cdots \mathrm{SO}\left(2 n_{k}\right) \mathrm{U}\left(m_{1}\right) \cdots \mathrm{U}\left(m_{l}\right) \\
\text { where } k+\sum n_{i}+\sum m_{i}=n\end{array}$ \\
\hline $\mathrm{Sp}(n)$ & $\begin{array}{c}\mathrm{U}\left(n_{1}\right) \cdots \mathrm{U}\left(n_{k}\right) \mathrm{Sp}\left(m_{1}\right) \cdots \mathrm{Sp}\left(m_{l}\right) \\
\text { where } \sum n_{i}+\sum m_{i}=m\end{array}$ \\
\hline
\end{tabular}

Table 15: Connected subgroups $\mathrm{K}_{+}$of maximal rank of the simple classical Lie groups

\begin{tabular}{|c|c|c|c|}
\hline Dimension & $\mathbf{K}$ & $\mathbf{H}$ & Isotropy representation \\
\hline $\mathrm{n}$ & $\mathrm{SO}(n+1)$ & $\mathrm{SO}(n)$ & $\rho_{n}$ \\
\hline $2 \mathrm{n}+1$ & $\mathrm{SU}(n+1)$ & $\mathrm{SU}(n)$ & $\mu_{n} \oplus i d$ \\
\hline $2 \mathrm{n}+1$ & $\mathrm{U}(n+1)$ & $\mathrm{U}(n)$ & $\mu_{n} \oplus i d$ \\
\hline $4 \mathrm{n}+3$ & $\mathrm{Sp}(n+1)$ & $\mathrm{Sp}(n)$ & $\nu_{n} \oplus 3 i d$ \\
\hline $4 \mathrm{n}+3$ & $\mathrm{Sp}(n+1) \mathrm{Sp}(1)$ & $\mathrm{Sp}(n) \Delta \mathrm{Sp}(1)$ & $\nu_{n} \hat{\otimes} \nu_{1} \oplus i d \hat{\oplus} \rho_{3}$ \\
\hline $4 \mathrm{n}+3$ & $\mathrm{Sp}(n+1) \mathrm{U}(1)$ & $\mathrm{Sp}(n) \Delta \mathrm{U}(1)$ & $\nu_{n} \hat{\otimes} \phi \oplus i d \hat{\otimes} \phi \oplus i d$ \\
\hline 15 & $\mathrm{Spin}(9)$ & $\mathrm{Spin}(7)$ & $\rho_{7} \oplus \Delta_{8}$ \\
\hline 7 & $\mathrm{Spin}(7)$ & $\mathrm{G}_{2}$ & $\phi_{7}$ \\
\hline 6 & $\mathrm{G}_{2}$ & $\mathrm{SU}(3)$ & $\mu_{3}$ \\
\hline
\end{tabular}

Table 16: Transitive effective actions on the sphere $S^{n}$ 


\section{B Some computations}

\section{B.1 The normalizer of $\mathrm{U}(n)$ in $\mathrm{SO}(2 n)$}

The group $\mathrm{U}(n)$ is given as the centralizer of an element in $\mathrm{SO}(2 n)$ of order 4, which is unique up to sign, and the standard $\mathrm{U}(n)$ is given by the matrix $J$ containing $\left(\begin{array}{cc}0 & 1 \\ -1 & 0\end{array}\right)$ on the diagonal 2x2-blocks, and 0 everywhere else. Let $p=\operatorname{diag}(-1,1,-1,1, \ldots)$, so that conjugation with $p$ corresponds to complex conjugation on $\mathrm{U}(n)$, and let $N$ be the normalizer of $\mathrm{U}(n)$ in $\mathrm{SO}(2 n)$. An element $g \in \mathrm{SO}(2 n)$ is in $N$ if and only if for all $u \in \mathrm{U}(n)$ the element $g u g^{-1}$ is in $\mathrm{U}(n)$ again, i.e. commutes with $J$ :

$$
g \in N \Leftrightarrow g u g^{-1} J=J g u g^{-1} \forall u \in \mathrm{U}(n) \Leftrightarrow u g^{-1} J g=g^{-1} J g u \forall u \in \mathrm{U}(n)
$$

The last equation says that $g^{-1} J g$ is in the centralizer of $\mathrm{U}(n)$, which is its center. But $g^{-1} J g$ is of order 4, so it's either $J$ or $-J$. By $\mathrm{U}(n)$ being connected, we have

$$
N=\left\{g \in \mathrm{SO}(2 n) \mid g^{-1} J g=J\right\} \cup\left\{g \in \mathrm{SO}(2 n) \mid g^{-1} J g=-J\right\}
$$

If $n$ is odd, the second set is empty by looking at the determinant, so we have $N=\mathrm{U}(n)$. If $n$ is even, one easily sees that $p$ satisfies $p J p=-J$, so we have

$$
N=\mathbb{Z}_{2} \cdot \mathrm{U}(n)=\mathrm{U}(n) \cup p \mathrm{U}(n)
$$

\section{B.2 Sphere actions of $\mathrm{U}(n)$}

\section{B.2.1 Actions of $\mathrm{U}(n+1)$ on spheres}

The group $\mathrm{U}(n+1)$ is the quotient of $\mathrm{S}^{1} \times \mathrm{SU}(n+1)$ by the subgroup $C_{n+1}$ of $n$ th roots of unity (embedded diagonally), so the representations of $\mathrm{U}(n+1)$ are exactly the representations of $\mathrm{S}^{1} \times \mathrm{SU}(n)$ with kernel $C_{n+1}$. The irreducible representations of $\mathrm{S}^{1} \times \mathrm{SU}(n+1)$ are the tensor products of irreducible representations of $\mathbf{S}^{1}$ and $\mathbf{S U}(n+1)$ respectively. Since any action on a sphere is linear, it gives rise to an irreducible representation of $\mathrm{U}(n+1)$, and checking through the possibilities of table 16 on the preceding page, it is clear that we are looking for irreducible representations of real dimension 2 or $2 n+2$.

Representations of real dimension 2 of $\mathrm{S}^{1} \times \mathrm{SU}(n+1)$ are given as $\phi_{m} \hat{\otimes} \rho_{0}$, where $\phi_{m}$ is the representation of $\boldsymbol{S}^{1}$ on $\mathbb{C}$ given by $(z, v) \mapsto z^{m} v$. Requiring the kernel to be $C_{n+1}$ leads to the condition that $n$ divides $m$, or $m=k(n+1)$ for some $k \in \mathbb{Z}$. This is $\mathrm{U}(n+1)$ acting on $\mathbb{C}$ via $(A, v) \mapsto(\operatorname{det} A)^{k} v$. 
Since all irreducible representations of $S^{1}$ are complex of dimension 1 , we find the irreducible representations of dimension $2 n+2$ of $\mathrm{S}^{1} \times \mathrm{SU}(n+1)$ by looking for the representations of $\mathrm{SU}(n+1)$ that are either of dimension $n+1$ and irreducible or that are of dimension $2 n+2$ and possess a real structure. The first case leads to the standard representation $\mu_{1}$, which in turn gives the representation $\phi_{m} \hat{\otimes} \mu_{1}$ of $\mathrm{S}^{1} \times \mathrm{SU}(n+1)$. Requiring this to be a representation of $\mathrm{U}(n+1)$ leads to constraints on $m$ as above and results in the representation $(A, v) \mapsto(\operatorname{det} A)^{k} A v$ on $\mathbb{C}^{n+1} \simeq \mathbb{R}^{2 n+2}$. As for the second case, the only representation of $\mathrm{SU}(n+1)$ of dimension $2 n+2$ possessing a real structure is $\mu_{1} \oplus \bar{\mu}_{1}$ (for $n \geq 5$ this is clear for reasons of dimension; in the lower-dimensional cases some extra representations have to be checked from the tables). This leads to an irreducible representation on $\mathbb{R}^{2 n+2}$, which is the same as the one we obtained from the first case.

\section{B.2.2 Identifying the $\mathrm{S}^{1}$}

From the previous subsection we know that the actions of $\mathrm{U}(n)$ on spheres of greater dimension than 1 are given in the following way: $A \in \mathrm{U}(n)$ acts by $(\operatorname{det} A)^{k} A$. It is easily seen that the isotropy group of such an action is given by

$$
\left\{\left(\begin{array}{cccc}
a & 0 & 0 & \cdots \\
0 & & & \\
0 & A & \\
\vdots & & &
\end{array}\right) \mid A \in \mathbb{C}, A \in \mathrm{U}(n-1), a^{k+1}=\overline{\operatorname{det} A}^{k}\right\}
$$

Also one easily verifies that this is $\mathrm{S}_{k}^{1} \mathrm{SU}(n-1)$ where

$$
\mathrm{S}_{k}^{1}=\left\{\operatorname{diag}\left(z^{(k+1)(n-1)}, z^{-k}, \ldots, z^{-k}\right) \mid z \in \mathrm{S}^{1}\right\}
$$

Replace $z$ by $z^{n}$ and write this as a product of

$$
\operatorname{diag}\left(z^{n-1}, z^{n-1}, \ldots, z^{n-1}\right)
$$

and

$$
\operatorname{diag}\left(z^{(k+1)(n-1) n-(n-1)}, z^{-k n-(n-1)}, \ldots, z^{-k n-(n-1)}\right)
$$

where the first is an element of the center and the second in $\operatorname{SU}(n)$, so one can figure out the isotropy group if the group is embedded in a larger one. 


\section{B.2.3 Actions of $\mathrm{U}(n)$ on real projective spaces}

If $\mathrm{U}(n)$ acts transitively almost effectively on the real projective space $\mathbb{R} P^{2 n-1}$, the isotropy group is given by $\mathrm{S}_{l, k}^{1} \mathrm{SU}(n-1)$, where

$$
\mathrm{S}_{l, k}^{1}=\left\{\operatorname{diag}\left(z^{l}, z^{-k}, \ldots, z^{-k}\right) \mid z \in \mathrm{S}^{1}\right\}, \operatorname{gcd}(k, l)=1
$$

We want to show $l=(k+2)(n-1)$.

To prove that, we look at the map $\pi_{1}\left(\mathrm{~S}_{l, k}^{1}\right) \rightarrow \pi_{1}(\mathrm{U}(n))$ induced by inclusion. We have $\pi_{1}\left(\mathbb{R} P^{2 n-1}\right)=\mathbb{Z}_{2}$, so the image has index 2 in the fundamental group of $\mathrm{U}(n)$. We know $\mathrm{S}_{l, k}^{1}$ is homotopic to

$$
\left\{\operatorname{diag}\left(z^{l}, z^{-k(n-1)}, 1 \ldots, 1\right)\right\}=\left\{\operatorname{diag}\left(z^{\frac{l}{a}}, z^{-k \frac{n-1}{a}}, 1, \ldots, 1\right)\right\}
$$

where $a=\operatorname{gcd}(l, n-1)$. This in turn is homotopic to

$$
\left\{\operatorname{diag}\left(z^{\frac{l}{a}-k \frac{n-1}{a}}, 1, \ldots, 1\right)\right\}
$$

By what we said above, it is clear that $\left|\frac{l}{a}-k \frac{n-1}{a}\right|=2$. First suppose $\frac{l}{a}-k \frac{n-1}{a}=2$. Then $\frac{l}{a}=k \frac{n-1}{a}+2$, and we consider the following sequence (where homotopy is denoted by $\simeq$ ):

$$
\begin{aligned}
& \left\{\operatorname{diag}\left(z^{k \frac{n-1}{a}}, z^{-k \frac{n-1}{a}}, 1, \ldots, 1\right)\right\} \\
= & \left\{\operatorname{diag}\left(z^{\left(k \frac{n-1}{a}+2\right)(n-1)}, z^{-k(n-1) \frac{n-1}{a}}, 1, \ldots, 1\right)\right\} \\
\simeq & \left\{\operatorname{diag}\left(z^{\left(k \frac{n-1}{a}+2\right)(n-1)}, z^{-k \frac{n-1}{a}}, z^{-k \frac{n-1}{a}}, \ldots, z^{-k \frac{n-1}{a}}\right)\right\}
\end{aligned}
$$

By replacing $k$ by $k \frac{n-1}{a}$, we arrive at the aforementioned form. A similar computation shows the same if $\frac{l}{a}-k \frac{n-1}{a}=-2$.

The converse of the claim is also clear from the above computation: The index of the image of $\mathrm{S}_{l, k}^{1}$ in the fundamental group of $\mathrm{U}(n)$ has index 2 , if $l=(k+2)(n-1)$ and $\operatorname{gcd}(l, k)=1$. 


\section{References}

Riemannian manifolds of cohomogenity one. In János Szenthe, editor, Differential geometry and its applications, pages 9-22. Amsterdam [u.a.], 1992.

A. V. Alekseevsky and D.V. Alekseevsky. G-manifolds with one dimensional orbit space. In Ernest B. Vinberg, editor, Lie groups, their discrete subgroups, and invariant theory, pages 1-31. Providence, R.I., 1992.

A. V. Alekseevsky and D.V. Alekseevsky. Riemannian g-manifolds with one dimensional orbit space. Annals of global analysis and geometry, pages 197-211, 1993.

D.V. Alekseevsky and F. Podestá. Compact cohomogeneity one Riemannian manifolds of positive Euler characteristic and quaternionic Kähler manifolds. In Boris N. Apanasov, editor, Geometry, topology and physics, pages 1-33. Berlin [u.a.], 1997.

G. E. Bredon. Introduction to Compact Transformation Groups. Academic Press, 1972.

M. Goto and F.D. Grosshans. Semisimple Lie algebras. Lecture notes in Pure and Appl. Math. Marcel Dekker Inc, 38 edition, 1978.

K. Grove, L. Verdiani, B. Wilking, and W. Ziller. Non-negative curvature obstructions in cohomogeneity one and the Kervaire spheres. Ann. Sc. Norm. Super. Pisa Cl. Sci., 5(5) no. 2:159-170, 2006.

K. Grove, B. Wilking, and W. Ziller. Positively curved cohomogeneity one manifolds and 3-Sasakian manifolds. J. Differential Geom., 78(1):33-111, 2008.

Karsten Grove and Wolfgang Ziller. Curvature and symmetry of Milnor spheres. Annals of Mathematics, 152:331-367, 2000.

Karsten Grove and Wolfgang Ziller. Cohomogeneity one manifolds with positive Ricci curvature. Inv. Math., 149:619-646, 2002.

C. Hoelscher. Classification of cohomogeneity one manifolds in low dimensions. Dissertation, University of Pennsylvania, 2007. URL: http://www.math.rutgers.edu/ coreyah/dissertation.pdf.

Paul S. Mostert. On a compact lie group acting on a manifold. The Annals of Mathematics, 65:447-455, 1957. 
Walter D. Neumann. 3-dimensional G-manifolds with 2-dimensional orbits. In Proc. Conf. on Transformation Groups, pages 220-222, New York, 1968. Springer.

J. Parker. 4-dimensional G-manifolds with 3-dimensional orbits. Pacific Journal of Mathematics, 125 (1):187-204, 1986. 